Proceedings of the

International Geometry Center

Vol. 13, no. 4 (2020) pp. 125-178

\title{
Incidence coefficients in the Novikov Complex for Morse forms: rationality and exponential growth properties
}

\author{
Andrei Pajitnov \\ Dedicated to the memory of $V$. V. Sharko
}

\begin{abstract}
Let $f: M \rightarrow S^{1}$ be a Morse map, $v$ a transverse $f$-gradient. The construction of the Novikov complex associates to these data a free chain complex $C_{*}(f, v)$ over the ring $\mathbb{Z}[[t]]\left[t^{-1}\right]$, generated by the critical points of $f$. This complex computes the completed homology module of the corresponding infinite cyclic covering of $M$. Novikov's Exponential Growth Conjecture says that the boundary operators in this complex are power series of non-zero convergence radius (see [10]).

In [14] the author announced the proof of the Novikov conjecture for the case of $C^{0}$-generic gradients together with several generalizations. The proofs of the first part of this work were published in [15], see also [16].

The present article contains the proofs of the second part.

There is a refined version of the Novikov complex, defined over a suitable completion of the group ring of the fundamental group. We prove that for a $C^{0}$-generic $f$-gradient the corresponding incidence coefficients belong to the image in the Novikov ring of a (non commutative) localization of the fundamental group ring.

The Novikov construction generalizes also to the case of Morse 1-forms. In this case the corresponding incidence coefficients belong to a certain completion of the ring of integral Laurent polynomials of several variables. We prove that for a given Morse form $\omega$ and a $C^{0}$-generic $\omega$-gradient these incidence coefficients are rational functions.

The incidence coefficients in the Novikov complex are obtained by counting the algebraic number of the trajectories of the gradient, joining the zeros of the Morse form. There is V. I. Arnold's version of the exponential growth conjecture, which concerns the total number of trajectories. We confirm this
\end{abstract}

Keywords: Novikov complex, non-commutative localization, exponential growth conjecture

Ключові слова: Комплекс Новікова, некомутативна локалізація, гіпотеза експоненційного росту

DOI: http://dx.doi.org/10.15673/tmgc.v13i4.1747 
stronger form of the conjecture for any given Morse form and a $C^{0}$-dense set of its gradients.

We give an example of explicit computation of the Novikov complex.

Анотація. Нехай $f: M \rightarrow S^{1}$ - відображення Морса і $v$ - $f$-градієнтне векторне поле. Комплекс Новікова $C_{*}(f, v)$ - це вільний ланцюговий комплекс над кільцем $\mathbb{Z}[[t]]\left[t^{-1}\right]$, породжений критичними токами $f$. Він дозволяє обчислити поповнений модуль гомологій відповідного нескінченного циклічного накриття $M$. Гіпотеза Новікова про експоненційний зріст стверджує що граничні оператори цього комплекса є степеневими рядами з ненульовими радіусами збіжності (див. [10]).

В роботі [14] анонсовано доведення гіпотези Новікова для випадку $C^{0}$-типових градієнтний векторних полів та деякі узагальнення цієї гіпотези. Доведення першої частини вказаних результатів опубліковано в [15], див. також [16]. Представлена робота містить доведення другої частини [14].

Існує спрощена версія комплекса Новікова, визначена над певним поповненням групового кільця фундаментальної групи. Ми показуємо, що для $C^{0}$-типових $f$-градієнтних векторних полів відповідні коефіцієнти інцидентності належать образу кільця Новікова (некомутативної) локалізації фундаметального груповаого кільця.

Конструкція Новікова також узагальнюється на випадок морсівських 1-форм. В цій ситуації відповідні коефіцієнти інцидентності належать поповненню кільця поліномів Лорана від кількох змінних з цілими коєфіцієнтами. Ми доводимо, шр для заданої морсівської форми $\omega$ і $C^{0}$ типового $\omega$-градієнтного векторного поля ці коєфіцієнти інцидентності є раціональними функціями.

Коєфіцієнти інцидентності в комплексі Новікова можна отримати обчислюючи алгебраїчне число траєкторій градієнтного векторного поля, що з'єднують нулі даної форми. Існує версія В. І. Арнольда гіпотези експоненційного росту про число таких траєкторій. В роботі доведено сильну форму цієї гіпотези для кожної морсівської форми та $C^{0}$-щільної множини градієнтних векторних полів.

Ми також наводимо приклади конкретних обчислень комплекса Новікова.

\section{INTRODUCTION}

Let $f: M \rightarrow S^{1}$ be a Morse map, $v$ a transverse $f$-gradient. Denote by $\bar{M} \rightarrow M$ the infinite cyclic covering corresponding to $f$. The classical construction of the Morse chain complex of a real-valued Morse function can be generalized to this case; one obtains the Novikov complex $C_{*}(f, v)$ defined over the ring $\mathbb{Z}[[t]]\left[t^{-1}\right]$ of Laurent power series with integer coefficients and finite negative part. The homology of this chain complex is isomorphic to a certain completion of the homology module $H_{*}(\bar{M})$. Novikov's Exponential Growth Conjecture says that the boundary operators in $C_{*}(f, v)$ are power 
series of non-zero convergence raduis. In 1995 the author proved that these boundary operators are rational functions of $t$ for the case of $C^{0}$-generic gradient $v$. That implies the Novikov Exponential Growth Conjecture for $C^{0}$-generic gradients. This result and its generalizations were announced in [14], and the proofs of first part of these results were published in [15]. The proofs of the second part were available as an e-print [13]. Since then the results of this e-print were used in several papers, see e.g. [5]. Recently this domain attracted again the interest of researchers, see the article [6], where a family of examples of Novikov complex with infinite power series as incidence coefficients was constructed.

The present paper is a revised version of the eprint [13]. Several references were updated, and references to more recent articles were added. Thus this article completes the publication of the results announced in [14].

The paper falls naturally into three parts. The first part (Section 2) contains a recollection of the material of the author's paper [15] in a compressed and rearranged form, suitable for our present purposes. The second part (Section 3,4) is the hard core of the paper. We prove here that the rationality property of the boundary operators is $C^{0}$-generic ${ }^{1}$. In Section 3 we deal with non-abelian Novikov rings and the rationality property is expressed in terms of a certain non-abelian localization of the group ring of the fundamental group of the manifold. In Section 4 we consider the case of arbitrary Morse forms and free abelian coverings.

In the third part we study the exponential growth properties. We begin by an example of a Novikov complex where the incidence coefficients grow exponentially (Section 5). Then we apply the results of the previous sections to show that the exponential growth property of the incidence coefficients of the gradient of a Morse form is $C^{0}$-generic.

In the rest of the Introduction we give the statement of our main results preceded by short background subsections 1.1 and 1.2.

1.1. Morse-Novikov theory for circle-valued Morse functions. The classical Morse-Thom-Smale construction associates to a Morse function $g: M \rightarrow \mathbb{R}$ on a closed manifold a free chain complex $C_{*}(g)$ where the number $m\left(C_{p}(g)\right)$ of free generators of $C_{p}(g)$ equals the number of the critical points of $g$ of index $p$ for each $p$. The boundary operator in this complex is defined in a geometric way, counting the trajectories of a gradient of $g$, joining critical points of $g$ (see $[8,12,17-19]$ ).

In the early $80 \mathrm{~s}$ S. P. Novikov generalized this construction to the case of maps $f: M \rightarrow S^{1}$ (see [9]). The corresponding analogue of Morse complex is a free chain complex $C_{*}(f)$ over $\mathbb{Z}[[t]]\left[t^{-1}\right]$. Its number of free generators

\footnotetext{
${ }^{1}$ For the precise meaning of $C^{0}$-generisity in our context see the statements of the Theorems A, B, C
} 
equals the number of critical points of $f$ of index $p$, and the homology of $C_{*}(f)$ equals to the completed homology of the cyclic covering.

Fix some $k$. The boundary operator $\partial: C_{k}(f) \rightarrow C_{k-1}(f)$ is represented by a matrix, which entries are in the ring of Laurent power series. That is $\partial_{i j}=\sum_{n=-N}^{\infty} a_{n} t^{n}$, where $a_{n} \in \mathbb{Z}$, and $N$ is some natural number.

Since the beginning S. P. Novikov conjectured that the power series $\partial_{i j}$ have some nice analytic properties. In particular he conjectured that:

- generically the coefficients $a_{n}$ of $\partial_{i j}=\sum_{n=-N}^{\infty} a_{n} t^{n}$ grow at most exponentially with $n$.

In [15] we have proved that for a $C^{0}$ generic $f$-gradient the incidence coefficients above are actually rational functions. To recall the statement of the Main Theorem of [15] let $M$ be a closed connected manifold and $f: M \rightarrow S^{1}$ a Morse map, non-homotopic to zero. Denote the set of critical points of $f$ by $S(f)$. The set of $f$-gradients of the class $C^{\infty}$, satisfying the transversality assumption (see $\S 1$ for terminology), will be denoted by $\mathcal{G} t(f)$. By Kupka-Smale theorem it is residual in the set of all the $C^{\infty}$ gradients. Choose $v \in \mathcal{G} t(f)$. Denote by $\bar{M} \stackrel{\mathcal{P}}{\rightarrow} M$ the connected infinite cyclic covering for which $f \circ \mathcal{P}$ is homotopic to zero. Choose a lift $F: \bar{M} \rightarrow \mathbb{R}$ of $f \circ \mathcal{P}$ and let $t$ be the generator of the structure group of $\mathcal{P}$ such that $F(x t)<F(x)$. The $t$-invariant lift of $v$ to $\bar{M}$ will be denoted by the same letter $v$. For every critical point $x$ of $f$ choose a lift $\bar{x}$ of $x$ to $\bar{M}$. Choose orientations of stable manifolds of critical points. Then for every $x, y \in S(f)$ with ind $x=$ ind $y+1$ and every $k \in \mathbb{Z}$ the incidence coefficient $n_{k}(x, y ; v)$ is defined (as the algebraic number of $(-v)$-trajectories joining $\bar{x}$ to $\bar{y} t^{k}$ ).

Theorem. ([15, p.971]) In the set $\mathcal{G t}(f)$ there is a subset $\mathcal{G} t_{0}(f)$ with the following properties.

(1) $\mathcal{G} t_{0}(f)$ is open and dense in $\mathcal{G} t(f)$ with respect to $C^{0}$ topology.

(2) If $v \in \mathcal{G} t_{0}(f), x, y \in S(f)$ and ind $x=$ ind $y+1$, then

$$
\sum_{k \in \mathbb{Z}} n_{k}(x, y ; v) t^{k}
$$

is a rational function of $t$ of the form $\frac{P(t)}{t^{m} Q(t)}$, where $P(t)$ and $Q(t)$ are polynomials with integral coefficients, $m \in \mathbb{N}$, and $Q(0)=1$.

(3) Let $v \in \mathcal{G} t_{0}(f)$ and $U$ be a neighborhood of $S(f)$. Then for every $w \in \mathcal{G} t_{0}(f)$ such that $w=v$ in $U$ and $w$ is sufficiently close to $v$ in $C^{0}$ topology we have: $n_{k}(x, y ; v)=n_{k}(x, y ; w)$ for every $x, y \in S(f), k \in \mathbb{Z}$.

The present paper uses the terminology and definitions of the paper [15], and should be read as a follow up of [15]. 
1.2. Morse forms and Novikov rings. To state our results, we recall some algebraic and Morse-theoretic definitions. Let $G$ be a group and

$$
\xi: G \rightarrow \mathbb{R}
$$

be a group homomorphism. We denote by $(\mathbb{Z} G)^{\wedge}$ the abelian group of all formal linear combinations $\sum_{g \in G} n_{g} g$ (infinite in general). Recall that the Novikov ring $\mathbb{Z} G_{\xi}^{-}$is the subring of all $\lambda \in(\mathbb{Z} G)^{\wedge}$ such that for every $c \in \mathbb{R}$ the set $\operatorname{supp} \lambda \cap \xi^{-1}([c, \infty))$ is finite.

Let $\omega$ be a closed 1-form on a manifold $M$. The de Rham cohomology class of $\omega$ will be denoted by $[\omega]$ and the corresponding homomorphism $\pi_{1} M \rightarrow \mathbb{R}$ will be denoted by $\{\omega\}$. We say that $\omega$ is a Morse form, if locally it is the differential of a Morse function. If $f: M \rightarrow S^{1}$ is a Morse map, then its differential is a Morse form, which cohomology class is in $H^{1}(M, \mathbb{Z})$. A Morse form $\omega$ is proportional to a differential of a Morse map $M \rightarrow S^{1}$ if and only if there exists $\lambda \in \mathbb{R}$ such that $\lambda[\omega] \in H^{1}(M, \mathbb{Z})$.

The terminology of $[15, \S 1.1]$ (which concerns Morse functions) is extended in an obvious way to the case of Morse forms, and we shall make free use of it. In particular we shall assume the notion of $\omega$-gradient. (See Subsection 2.1 for a detailed recollection.) The set of all $\omega$-gradients, satisfying the transversality assumption will be denoted by $\mathcal{G} t(\omega)$.

Let $\omega$ be a Morse form on a closed connected manifold $M, v \in \mathcal{G} t(\omega)$, and $x, y \in S(\omega)$ with ind $x=$ ind $y+1$. Choose some lifts $\widetilde{x}, \widetilde{y}$ of $x, y$ to $\widetilde{M}$ and some orientations of the stable manifolds of $x$ and of $y$. In Subsection 4.2 we define the incidence coefficient $\tilde{n}(\tilde{x}, \tilde{y} ; v) \in \mathbb{Z}\left(\pi_{1} M\right)_{\xi}^{-}$(Definition 4.2.5).

\subsection{Statement of the results.}

1.3.1. Morse maps $M \rightarrow S^{1}$. Let $\xi: G \rightarrow \mathbb{Z}$ be a group epimorphism. Denote $\operatorname{Ker} \xi$ by $H$. For $n \in \mathbb{Z}$ denote

- $\xi^{-1}(n)$ by $G_{(n)}$;

- $\left\{x \in \mathbb{Z} G \mid \operatorname{supp} x \subset G_{(n)}\right\}$ by $\mathbb{Z} G_{(n)}$;

- $\xi^{-1}((-\infty,-1])$ by $G_{-}$;

- and $\left\{x \in \mathbb{Z} G \mid \operatorname{supp} x \subset G_{-}\right\}$by $\mathbb{Z} G_{-}$.

Choose $\theta \in \mathbb{Z} G_{(-1)}$. It is easy to see that $\mathbb{Z} G_{\xi}^{-}$is identified with the ring of power series of the form $\left\{a_{-n} \theta^{-n}+\ldots+a_{1} \theta+\ldots \mid a_{i} \in \mathbb{Z} H\right\}$. Put

$$
\Sigma_{n}=\left\{\mathbf{1}+A \mid A \in \operatorname{Mat}_{n \times n}\left(\mathbb{Z} G_{(-1)}\right)\right\}, \quad \Sigma=\bigcup_{n \geqslant 1} \Sigma_{n} .
$$

There is the corresponding localization ring $\mathbb{Z}_{\Sigma}$ (see [4, p. 291]). Every matrix in $\Sigma_{n}$ is invertible in $\operatorname{Mat}_{n \times n}\left(\mathbb{Z} G_{\xi}^{-}\right)$, the inverse of $\mathbf{1}+A$ being given 
by $\sum_{n=0}^{\infty}(-1)^{n} A^{n}$, therefore the localization map $\lambda: \mathbb{Z} G \rightarrow \mathbb{Z} G_{\Sigma}$ is injective and the inclusion $i: \mathbb{Z} G \hookrightarrow \mathbb{Z} G_{\xi}^{-}$factors through a ring homomorphism $\ell: \mathbb{Z} G_{\Sigma} \rightarrow \mathbb{Z} G_{\xi}^{-}$.

Let $M$ be a connected closed manifold and $f: M \rightarrow S^{1}$ be a Morse map, non-homotopic to zero. Let $G=\pi_{1}(M)$ and denote by $\xi$ the induced homomorphism $G \rightarrow \mathbb{Z}$. Denote by $p: \widetilde{M} \rightarrow M$ the universal covering of $M$.

Theorem A. In the set $\mathcal{G} t(f)$ there is a subset $\mathcal{G} t_{1}(f)$ with the following properties:

(1) $\mathcal{G} t_{1}(f)$ is open and dense in $\mathcal{G} t(f)$ with respect to $C^{0}$ topology.

(2) If $v \in \mathcal{G} t_{1}(f)$ then for every $x, y \in S(f)$ with ind $x=$ ind $y+1$ we have $\widetilde{n}(\widetilde{x}, \widetilde{y} ; v) \in \operatorname{Im} \ell$.

(3) Let $v \in \mathcal{G} t_{1}(f)$. Let $U$ be a neighborhood of $S(f)$. Then for every $w \in \mathcal{G} t_{1}(f)$ such that $w=v$ in $U$ and $w$ is sufficiently close to $v$ in $C^{0}$ topology we have: $\widetilde{n}(\widetilde{x}, \widetilde{y} ; v)=\widetilde{n}(\widetilde{x}, \widetilde{y} ; w)$ for every $x, y \in S(f)$.

1.3.2. Morse forms within arbitrary cohomology classes. At present we can prove the analogue of Theorem A in the case of arbitrary Morse forms only for the incidence coefficients associated with free abelian coverings.

Let $\omega$ be a Morse form on a closed connected manifold $M$. If $\phi: \widehat{M} \rightarrow M$ is any regular covering with the structure group $G$, such that $\phi^{*}([\omega])=0$, then the homomorphism $\{\omega\}: \pi_{1} M \rightarrow \mathbb{R}$ factors as $\pi_{1} M \rightarrow G \rightarrow \mathbb{R}$ and it is not difficult to see that the incidence coefficients $\widehat{n}(\widehat{x}, \widehat{y} ; v)$ are defined for every $v \in \mathcal{G} t(\omega)$ (here we suppose that ind $x=$ ind $y+1$, and that for every $p \in S(\omega)$ a lift $\hat{p}$ of $p$ to $\widehat{M}$ and an orientation of the stable manifold of $p$ are chosen). In particular, it is the case for the maximal free abelian covering $\overline{\bar{M}} \stackrel{\mathcal{P}}{\rightarrow} M$ with the structure group $H_{1}(M, \mathbb{Z}) /$ Tors $\approx \mathbb{Z}^{m}$. By abuse of notation we shall denote the corresponding homomorphism $\mathbb{Z}^{m} \rightarrow \mathbb{R}$ by the same symbol as the de Rham cohomology class $[\omega]$ of $\omega$. Assume that $[\omega] \neq 0$. Put

$$
\mathfrak{s}_{[\omega]}=\left\{P \in \mathbb{Z}\left[\mathbb{Z}^{m}\right] \mid P=\mathbf{1}+Q: \operatorname{supp} Q \subset[\omega]^{-1}((-\infty, 0))\right\} .
$$

Then $\mathfrak{s}[\omega]$ is a multiplicative subset of $\mathbb{Z}\left[\mathbb{Z}^{m}\right]$.

Theorem B. There is a subset $\mathcal{G} t_{1}(\omega) \subset \mathcal{G} t(\omega)$ with the following properties:

(1) $\mathcal{G} t_{1}(\omega)$ is open and dense in $\mathcal{G} t(\omega)$ with respect to $C^{0}$ topology.

(2) For every $v \in \mathcal{G} t_{1}(\omega)$ and every $x, y \in S(\omega)$ with ind $x=$ ind $y+1$ we have: $\overline{\bar{n}}(\overline{\bar{x}}, \overline{\bar{y}} ; v) \in \mathfrak{s}_{[\omega]}^{-1} \mathbb{Z}\left[\mathbb{Z}^{m}\right]$. 
(3) Let $v \in \mathcal{G} t_{1}(\omega)$. Let $U$ be a neighborhood of $S(\omega)$. Then for every $w \in \mathcal{G} t_{1}(\omega)$ such that $w=v$ in $U$ and $w$ is sufficiently close to $v$ in $C^{0}$ topology we have: $\overline{\bar{n}}(\overline{\bar{x}}, \overline{\bar{y}} ; v)=\overline{\bar{n}}(\overline{\bar{x}}, \overline{\bar{y}} ; w)$ for every $x, y \in S(\omega), k \in \mathbb{Z}$.

1.3.3. An example. In Section 5 we construct a three-manifold $M$, a Morse map $f: M \rightarrow S^{1}$ and an $f$-gradient $v$ such that $n_{0}(\bar{x}, \bar{y} ; v)=0$ and for $k \geqslant 0$ we have

$$
n_{k+1}(\bar{x}, \bar{y} ; v)=-\frac{4}{\sqrt{5}} \cdot\left(\left(\frac{3+\sqrt{5}}{2}\right)^{k}-\left(\frac{3-\sqrt{5}}{2}\right)^{k}\right) .
$$

1.3.4. Exponential growth estimates. The Novikov Exponential Growth conjecture [10] has a natural generalization to the case of Morse forms of arbitrary irrationality degree. Let $\omega$ be a Morse form and $\mathcal{P}: \overline{\bar{M}} \rightarrow M$ be the maximal free abelian covering. Let $F: \overline{\bar{M}} \rightarrow \mathbb{R}$ be a function such that $d F=\mathcal{P}^{*}([\omega])$. For critical point $x, y$ of $F$ with ind $x=$ ind $y+1$ and $h \in \mathbb{Z}^{m}$ let $n(x, y ; h)$ be the algebraic number of $(-v)$-trajectories joining $x$ with $y h$. Then the generalized exponential growth conjecture says that there are constants $A, B>0$ such that for every $x, y$ with ind $x=$ ind $y+1$ and every $h \in \mathbb{Z}^{m}$ we have

$$
|n(x, y ; h)| \leqslant A \cdot e^{B[\omega](h)} .
$$

Theorem $\mathrm{C}$ below asserts a much stronger property, pertaining to the universal covering of $M$ and counting for every $g \in G=\pi_{1}(M)$ the absolute value of the algebraic number of trajectories joining $x$ with $y \cdot g$. To state this theorem we need one more definition. Let $G$ be a group. For an element $a=\sum n_{g} g \in \mathbb{Z} G$ we denote by $\|a\|$ the sum $\sum\left|n_{g}\right|$. Let $\xi: G \rightarrow \mathbb{R}$ be a homomorphism. For $\lambda=\sum_{g} n_{g} g \in \mathbb{Z} G_{\xi}^{-}$and $c \in \mathbb{R}$ we denote by $\lambda[c]$ the element $\sum_{\xi(g) \geqslant c} n_{g} g$ of $\mathbb{Z} G$ and we set $N_{c}(\lambda)=\|\lambda[c]\|$. We shall say that $\lambda$ is of exponential growth if there are $A, B \geqslant 0$ such that for every $c<0$ we have $N_{c}(\lambda) \leqslant A e^{-c B}$. It is easy to prove that the elements of exponential growth form a subring of $\mathbb{Z} G_{\xi}^{-}$containing $\mathbb{Z} G$.

Theorem C. Let $v$ be an $\omega$-gradient, belonging to $\mathcal{G} t_{1}(\omega)$, and $x, y \in S(\omega)$ with ind $x=$ ind $y+1$. Then $\tilde{n}(\tilde{x}, \tilde{y} ; v) \in \mathbb{Z}\left[\pi_{1} M\right]_{\{\omega\}}^{-}$is of exponential growth.

In the paper [3, Theorem 2.21] D. Burghelea and S. Haller also prove that the property (1.1) holds for $C^{0}$-generic gradients ${ }^{2}$.

\footnotetext{
${ }^{2}$ The author thanks the anonymous referee for this information.
} 
1.3.5. Exponential estimates of absolute number of trajectories: Morse maps $M \rightarrow S^{1}$. The first published version of the Exponential Growth conjecture appeared in the paper of V. I. Arnold "Dynamics of intersections", 1989. This version is also the strongest one. Arnold writes in [1, p. 83]:

"The author is indebted to S. P. Novikov who has communicated the following conjecture, which was the starting point of the present paper. Let $p: \widetilde{M} \rightarrow M$ be a covering of a compact manifold $M$ with fiber $\mathbb{Z}^{n}$, and let $\alpha$ be a closed 1-form on $M$ such that $p^{*} \alpha=d f$, where $f: M \rightarrow \mathbb{R}$ is a Morse function. The Novikov conjecture states that, "generically" the number of the trajectories of the vector field $-\operatorname{grad} f$ on $M$ starting at a critical point $x$ of the function $f$ of index $k$ and connecting it with the critical points $y$ having index $k-1$ and satisfying $f(y) \geqslant f(x)-n$, grows in $n$ more slowly than some exponential, $e^{a n}$."

In the present paper we prove this conjecture for the $C^{0}$-dense subset in the set of all gradients (Theorems $\mathrm{C}$ and D below). We assume here the terminology of Subsection 1.3.1. The set of all $f$-gradients of class $C^{\infty}$ will be denoted by $\mathcal{G}(f)$. An $f$-gradient $v$ is called good if for every $p, q \in S(f)$ we have

$$
\text { (ind } p \leqslant \operatorname{ind} q+1) \Rightarrow(D(p, v) \pitchfork D(q,-v))
$$

The set of all good $f$-gradients will be denoted by $\mathcal{G} d(f)$. For $v \in \mathcal{G}(f)$ we denote by the same letter $v$ the $t$-invariant lift of $v$ to $\bar{M}$. Choose a lift

$$
F: \bar{M} \rightarrow \mathbb{R}
$$

of $f$ to $\bar{M}$. It is easy to prove that for $p, q \in S(F)$, ind $p=$ ind $q+1$ and for for $v \in \mathcal{G} d(f)$ the set of $(-v)$-trajectories, joining $p$ to $q$ is finite. The lifts of critical points of $f$ to $\bar{M}$ being chosen, denote by $N_{k}(x, y ; v)$ the number of $(-v)$-trajectories joining $\bar{x}$ to $\bar{y} t^{k}$ (where ind $x=$ ind $y+1$ ).

Theorem D. In the set $\mathcal{G}(f)$ there is a subset $\mathcal{G}_{0}(f)$ with the following properties:

(1) $\mathcal{G}_{0}(f)$ is $C^{0}$-dense in $\mathcal{G}(f)$ and $\mathcal{G}_{0}(f) \subset \mathcal{G} d(f)$.

(2) Let $v \in \mathcal{G}_{0}(f)$. Then there are constants $C, D>0$ such that for every $x, y \in S(f)$ with ind $x=$ ind $y+1$ and for every $k \in \mathbb{Z}$ we have $N_{k}(x, y ; v) \leqslant C \cdot D^{k}$.

1.3.6. Exponential estimates of absolute number of trajectories: Morse forms. Let $\omega$ be a Morse form on a closed connected manifold $M$. Let $\mathcal{P}: \overline{\bar{M}} \rightarrow M$ be the maximal free abelian covering of $M$ with structure group $\mathbb{Z}^{m}$; we identify the cohomology class $[\omega]$ of $\omega$ with the corresponding homomorphism $\mathbb{Z}^{m} \rightarrow \mathbb{R}$. We denote by $\mathcal{G}(\omega)$ the set of all $\omega$-gradients of class $C^{\infty}$ and by $\mathcal{G} d(\omega)$ the set of all good $\omega$-gradients of class $C^{\infty}$. Let 
$v \in \mathcal{G} d(\omega)$. For every zero $x$ of $\omega$ choose a lift $\overline{\bar{x}}$ of $x$ to $\overline{\bar{M}}$ and an orientation of the stable manifold of $x$. Then for every $g \in \mathbb{Z}^{m}$ and every $x, y \in S(\omega)$ with ind $x=$ ind $y+1$ the set of $(-v)$-trajectories joining $\bar{x}$ to $\overline{\bar{y}} g$ is finite and we denote its cardinality by $N(\overline{\bar{x}}, \overline{\bar{y}}, g ; v)$. For $c \in \mathbb{R}$ we denote by $N_{\geqslant c}(\overline{\bar{x}}, \overline{\bar{y}} ; v)$ the sum $\sum_{g:[\omega](g) \geqslant c} N(\overline{\bar{x}}, \overline{\bar{y}} ; g ; v)$.

Theorem E. In the set $\mathcal{G}(\omega)$ there is a subset $\mathcal{G}_{0}(\omega)$ with the following properties:

(1) $\mathcal{G}_{0}(\omega)$ is dense in $\mathcal{G}(\omega)$ with respect to $C^{0}$ topology; $\mathcal{G}_{0}(\omega) \subset \mathcal{G} d(\omega)$.

(2) Let $v \in \mathcal{G}_{0}(\omega)$. There exist constants $C, D>0$ such that for every $x, y \in S(\omega)$ with ind $x=$ ind $y+1$ and every $\lambda<0$ we have

$$
N_{\geqslant \lambda}(x, y ; v) \leqslant C \cdot D^{-\lambda} \text {. }
$$

Remark 1.3.7. Observe that Theorem D follows from Theorem E. We still keep both statements of Theorem D since the essential of the proof of Theorem E is based on the proof of Theorem D.

\section{RANGING SYSTEMS AND RANGING FLOWS (AN OVERVIEW OF RESULTS OF [15])}

The results of the present paper are based on the author's paper [15]. For the convenience of the reader we recall in this section some of the basic definitions and theorems of [15]. The contents of this section falls into two parts. In the first part (Subsections 2.2-2.3) we present the main technical tool for the rationality theorem, namely ranging systems. We show that if an $f$-gradient admit a ranging system adapted to a pair of critical points $p, q$, then the incidence coefficient of these two points is a rational function of the type described in the statement of the Main Theorem of [15]. The final result of this part is Theorem 2.3.4.

In the second part (Subsections 2.4-2.6) we show that the set of $f$ gradients that admit a ranging system adapted to $p, q$, is open and dense in the set of all transverse gradients (with respect to $C^{0}$-topology). The basic instruments here are the notions of ranging flow and ranging pair.

The exposition is preceded by a short introductory Subsection 2.1 where we collected the necessary definitions.

2.1. Terminology: functions and gradients. In the present subsection $M$ is a closed manifold.

Definition 2.1.1. Let $f: M \rightarrow \mathbb{R}$ be a Morse function on a closed manifold $M$. Denote $\operatorname{dim} M$ by $n$. The set of critical points of $f$ will be denoted by $S(f)$. A chart $\Phi_{p}: U_{p} \rightarrow B^{n}\left(0, r_{p}\right)$ (where $p \in S(f), U_{p}$ is a neighborhood 
of $p, r_{p}>0$ ) is called standard chart for $f$ around $p$ of radius $r_{p}$ (or simply $f$-chart) if there is an extension of $\Phi_{p}$ to a chart $\widetilde{\Phi}_{p}: V_{p} \rightarrow B^{n}\left(0, r_{p}^{\prime}\right)$, (where $\overline{U_{p}} \subset V_{p}$ and $r_{p}^{\prime}>r_{p}$ ), such that

$$
\left(f \circ \widetilde{\Phi}_{p}^{-1}\right)\left(x_{1}, \ldots, x_{n}\right)=f(p)+\sum_{i=1}^{n} \alpha_{i} x_{i}^{2},
$$

where $\alpha_{i}<0$ for $i \leqslant \operatorname{ind} p$ and $\alpha_{i}>0$ for $i>\operatorname{ind} p$. The domain $U_{p}$ is called standard coordinate neighborhood. Any such extension $\widetilde{\Phi}_{p}$ of $\Phi_{p}$ will be called standard extension of $\Phi_{p}$.

The set $\Phi_{p}^{-1}\left(\mathbb{R}^{k} \times\{0\}\right)$, resp. $\Phi_{p}^{-1}\left(\{0\} \times \mathbb{R}^{n-k}\right)$, where $k$ stands for ind $p$, is called negative disc, resp. positive disc. If for every $i$ we have $\alpha_{i}= \pm 1$, we shall say that the coordinate system $\left\{\Phi_{p}\right\}$ is strongly standard.

A family

$$
\mathcal{U}=\left\{\Phi_{p}: U_{p} \rightarrow B^{n}\left(0, r_{p}\right)\right\}_{p \in S(f)}
$$

of $f$-charts is called $f$-chart-system, if the family $\left\{\overline{U_{p}}\right\}$ is disjoint. We denote $\min _{p} r_{p}$ by $d(\mathcal{U})$, and $\max _{p} r_{p}$ by $D(\mathcal{U})$. If all the $r_{p}$ are equal to $r$, we shall say that $\mathcal{U}$ is of radius $r$.

The set $\Phi_{p}^{-1}\left(B^{n}(0, \lambda)\right)$, where $\lambda \leqslant r_{p}$ will be denoted by $U_{p}(\lambda)$. For $\lambda \leqslant d(\mathcal{U})$ we denote $\underset{p \in S(f)}{\cup} U_{p}(\lambda)$ by $\mathcal{U}(\lambda)$. Let

$$
\mathcal{U}=\left\{\Phi_{p}: U_{p} \rightarrow B^{n}\left(0, r_{p}\right)\right\}_{p \in S(f)}, \quad \mathcal{U}^{\prime}=\left\{\Phi_{p}^{\prime}: U_{p}^{\prime} \rightarrow B^{n}\left(0, r_{p}^{\prime}\right)\right\}_{p \in S(f)}
$$

be two $f$-chart-systems. We say, that $\mathcal{U}^{\prime}$ is a restriction of $\mathcal{U}$, if for every $p \in S(f)$ we have: $r_{p}^{\prime} \leqslant r_{p}, U_{p}^{\prime} \subset U_{p}, \Phi_{p}^{\prime}=\Phi_{p} \mid U_{p}^{\prime}$.

Given an $f$-chart system

$$
\mathcal{U}=\left\{\Phi_{p}: U_{p} \rightarrow B^{n}\left(0, r_{p}\right)\right\}_{p \in S(f)}
$$

we say, that a vector field $v$ on $M$ is an $f$-gradient with respect to $\mathcal{U}$, if

1) For every $x \in M \backslash S(f)$ we have $d f(v)(x)>0$;

2) For every $p \in S(f)$ we have

$$
\left(\widetilde{\Phi}_{p}\right)_{*}(v)=\left(-x_{1}, \ldots,-x_{k}, x_{k+1}, \ldots, x_{n}\right),
$$

where $k=$ ind ${ }_{f} p$, and $\widetilde{\Phi}_{p}$ is some standard extension of $\Phi_{p}$.

We say that a vector field $v$ is an $f$-gradient if there is an $f$-chart system $\mathcal{U}$, such that $v$ is an $f$-gradient with respect to $\mathcal{U}$.

Definition 2.1.2. Assume that $M$ is Riemannian and denote by $D_{r}(x)$, resp. $B_{r}(x)$ the closed, resp. open ball of radius $r$ centered in $x$. Let also $f: M \rightarrow \mathbb{R}$ be a Morse function, $v$ be an $f$-gradient, $p \in S(f)$. Set:

$$
\begin{aligned}
& B_{\delta}(p, v)=\left\{x \in M \mid \exists t \geqslant 0: \gamma(x, t ; v) \subset B_{\delta}(p)\right\}, \\
& D_{\delta}(p, v)=\left\{x \in M \mid \exists t \geqslant 0: \gamma(x, t ; v) \subset D_{\delta}(p)\right\},
\end{aligned}
$$




$$
D(p, v)=\left\{x \in M \mid \lim _{t \rightarrow \infty} \gamma(x, t ; v)=p\right\} .
$$

We denote by $K(v)$ the union of all subsets $D(p, v)$, and by $B_{\delta}(v)$ the union of all subsets $B_{\delta}(p, v)$ where $p$ ranges over critical points of $f$.

We also denote by $B_{\delta}($ ind $\leqslant s ; v)$ the union of subsets $B_{\delta}(p, v)$ (where $p$ ranges over critical points of $f$ of index $\leqslant s$ ). Similar notations like $D_{\delta}($ ind $\leqslant s ; v)$ or $K($ ind $=s ; v)$ etc. are now clear without special definition.

Remark 2.1.3. Definitions 2.1.1 and 2.1.2 generalize in an obvious way to the case of Morse functions on cobordisms and to the case of circle-valued Morse functions.

Definition 2.1.4. Let $f: W \rightarrow[a, b]$ be a Morse function on a compact Riemannian cobordism, $f^{-1}(b)=V_{1}, f^{-1}(a)=V_{0}, v$ be an $f$-gradient. For $x \in W$ we denote by $\gamma(x, t ; v)$ the value at $t$ of the integral curve of $v$ satisfying $\gamma(x, 0 ; v)=x$. If $x \in V_{1} \backslash K(-v)$, then the trajectory $\gamma(x, t ; v)$ reaches $V_{0}$ at some moment $t_{0}$; put

$$
\stackrel{m}{v}(x)=\gamma\left(x, t_{0} ; v\right) \text {. }
$$

We obtain thus a diffeomorphism

$$
\stackrel{m}{v}: V_{1} \backslash K(-v) \stackrel{\approx}{\rightarrow} V_{0} \backslash K(v) .
$$

Sometimes we denote it by $v_{[b, a]}^{m}$.

In the next section we take a closer look at this diffeomorphism.

2.2. Ranging systems. We proceed to the definition of ranging system (see [15, Definition 4.6]). Let $f: W \rightarrow[a, b]$ be a Morse function on a compact Riemannian cobordism, $f^{-1}(b)=V_{1}, f^{-1}(a)=V_{0}, v$ be an $f$ gradient.

Definition 2.2.1. Let $\Lambda=\left\{\lambda_{0}, \ldots, \lambda_{k}\right\}$ be a finite set of regular values of $f$, such that $\lambda_{0}=a, \lambda_{k}=b$, and for each $0 \leqslant i \leqslant k-1$ we have $\lambda_{i}<\lambda_{i+1}$ and there is exactly one critical value of $f$ in $\left[\lambda_{i}, \lambda_{i+1}\right]$. The values $\lambda_{i}, \lambda_{i+1}$ will be called adjacent. The set of pairs $\left\{\left(A_{\lambda}, B_{\lambda}\right)\right\}_{\lambda \in \Lambda}$ is called ranging system for $(f, v)$ if

(RS1) For every $\lambda \in \Lambda$ the sets $A_{\lambda}$ and $B_{\lambda}$ are disjoint compacts in $f^{-1}(\lambda)$.

(RS2) Let $\lambda, \mu \in \Lambda$ be adjacent. Then for every $p \in S(f) \cap f^{-1}([\lambda, \mu])$ one of the two following properties i), ii) holds:
i) $D(p, v) \cap f^{-1}(\lambda) \subset \operatorname{Int} A_{\lambda}$,
ii) $D(p,-v) \cap f^{-1}(\mu) \subset \operatorname{Int} B_{\mu}$. 
(RS3) Let $\lambda, \mu \in \Lambda$ be adjacent. Then

$$
v_{[\mu, \lambda]}^{m}\left(A_{\mu}\right) \subset \operatorname{Int} A_{\lambda}, \quad(-v)_{[\lambda, \mu]}^{\varkappa m}\left(B_{\lambda}\right) \subset \operatorname{Int} B_{\mu} .
$$

The existence of a ranging system allows us to endow the gradient flow with a structure that resembles to some extent a continuous map between compact spaces. The next proposition is proved in [15, Lemma 4.8 and Propositions 4.9, 4.10].

Proposition 2.2.2. Let $\left\{\left(A_{\lambda}, B_{\lambda}\right)\right\}_{\lambda \in \Lambda}$ be a ranging system for $(f, v)$. Let also $N$ be an oriented submanifold of $V_{1} \backslash B_{b}$ such that $N \backslash$ Int $A_{b}$ is compact. Then the following statements hold.

(1) There exists a homomorphism

$$
H(v): H_{*}\left(V_{1} \backslash B_{b}, A_{b}\right) \rightarrow H_{*}\left(V_{0} \backslash B_{a}, A_{a}\right),
$$

such that

$$
H(v)([N])=\left[v_{[b, a]}^{\varkappa}(N)\right]
$$

where $[N]$ is the fundamental class of $N$ in $H_{*}\left(V_{1} \backslash B_{b}, A_{b}\right)$ and $\left[v_{[b, a]}^{\rightsquigarrow}(N)\right]$ is the fundamental class of $v_{[b, a]}^{m}(N)$ in $H_{*}\left(V_{0} \backslash B_{a}, A_{a}\right)$.

(2) There is $\epsilon>0$ such that for any $f$-gradient $w$ with $\|v-w\|<\epsilon$ the ranging system $\left\{\left(A_{\lambda}, B_{\lambda}\right)\right\}_{\lambda \in \Lambda}$ is also a ranging system for $(f, w)$, and $H(v)=H(w)$.

Remark 2.2.3. Let $\lambda<\mu$ be elements of $\Lambda$. Similarly to Proposition 2.2 .2 one defines a homomorphism

$$
H(v)_{[\mu, \lambda]}: H_{*}\left(f^{-1}(\mu) \backslash B_{\mu}, A_{\mu}\right) \rightarrow H_{*}\left(f^{-1}(\lambda) \backslash B_{\lambda}, A_{\lambda}\right)
$$

which is $C^{0}$-stable with respect to small $C^{0}$-perturbations of $v$, as in property (2)of Proposition 2.2.2.

Ranging systems provide a convenient tool for computation of incidence coefficients in homology terms.

Definition 2.2.4. Let $\left\{\left(A_{\lambda}, B_{\lambda}\right)\right\}_{\lambda \in \Lambda}$ be a ranging system for

$$
f: W \rightarrow[a, b] .
$$

Let also $p \in S(f)$ and $\mu(p)$ and $\sigma(p)$ be the (uniquely determined) adjacent elements of $\Lambda$ such that $f(p) \in(\mu(p), \sigma(p))$. We will use the following notations:

- $S_{+}(p, v)$ denotes the sphere $D(p,-v) \cap f^{-1}(\sigma(p))$, and

- $S_{-}(p, v)$ denotes the sphere $D(p, v) \cap f^{-1}(\mu(p))$. 
If this does not lead to confusion, we denote these spheres simply by $S_{-}(p)$ and $S_{+}(p)$.

We say that a ranging system $\left\{\left(A_{\lambda}, B_{\lambda}\right)\right\}_{\lambda \in \Lambda}$ is adapted to a pair $p, q$ of critical points in $S(f)$, where ind $p=$ ind $q+1$, if

$$
S_{-}(p) \cap B_{\mu(p)}=\varnothing \quad \text { and } \quad S_{+}(q) \cap A_{\sigma(q)}=\varnothing .
$$

Let $l+1=$ ind $p$. Then ind $q=l$.

If $\left\{\left(A_{\lambda}, B_{\lambda}\right)\right\}_{\lambda \in \Lambda}$ is adapted to $p, q$, we denote by $\left[S_{-}(p)\right]$ also the image of this fundamental class in

$$
H_{l}\left(f^{-1}(\mu(p)) \backslash B_{\mu(p)}, A_{\mu(p)}\right) .
$$

Consider the inclusion

$$
j(v):\left(f^{-1}(\sigma(q)) \backslash B_{\sigma(q)}, A_{\sigma(q)}\right) \hookrightarrow\left(f^{-1}(\sigma(q)), f^{-1}(\sigma(q)) \backslash S_{+}(q)\right)
$$

and let $]] S_{+}(q, v)\left[\left[\right.\right.$ be the $(j(v))^{*}$-image of the class dual to $S_{+}(q)$ in the group $H^{l}\left(f^{-1}(\sigma(q)) \backslash B_{\sigma(q)}, A_{\sigma(q)}\right)$.

See the proof of the following two propositions in [15, Proposition 4.12].

Proposition 2.2.5. Let $\left\{\left(A_{\lambda}, B_{\lambda}\right)\right\}_{\lambda \in \Lambda}$ be a ranging system adapted to $p, q$. If $v$ is an $f$-gradient satisfying the transversality condition then

$$
n(p, q ; v)=\langle]] S_{+}(q)\left[\left[, H_{[\mu(p), \sigma(q)]}(v)\left(\left[S_{-}(p)\right]\right)\right\rangle .\right.
$$

This formula imply immediately a $C^{0}$-stability property for the incidence coefficients.

Proposition 2.2.6. (1) There is $\epsilon>0$ such that for every $f$-gradient $v$ with $\|w-v\|<\epsilon$ the system $\left\{\left(A_{\lambda}, B_{\lambda}\right)\right\}_{\lambda \in \Lambda}$ is a ranging system for $(f, w)$ adapted to $p, q$.

(2) Let $U(p)$ be a neighborhood of $p$, and $U(q)$ be a neighborhood of $q$. There is $\epsilon>0$ such that for every $f$-gradient $w$ with $\left.w\right|_{U(p)}=\left.v\right|_{U(p)}$ and $\left.w\right|_{U(q)}=\left.v\right|_{U(q)}$ and $\|w-v\|<\epsilon$ we have

$$
\left.\left.\left[S_{-}(p, w)\right]=\left[S_{-}(p, v)\right], \quad \quad\right]\right] S_{-}(q, w)[[=]] S_{-}(q, v)[[,
$$

and

$$
n(p, q ; v)=n(p, q ; w)
$$

In our applications the cobordism $W$ will be obtained as the result of cutting a closed manifold $M$ along a regular level surface of a Morse function $f: M \rightarrow S^{1}$. Such cobordism is endowed naturally with a diffeomorphism of its lower boundary on its upper boundary. 
Definition 2.2.7. A Riemannian cobordism $W$ with $\partial W=V_{0} \sqcup V_{1}$ is said to be cyclic if there is an isometry $\Phi: V_{0} \rightarrow V_{1}$ (such an isometry will be regarded as part of the structure of a cyclic cobordism).

Definition 2.2.8. A ranging system $\left\{\left(A_{\lambda}, B_{\lambda}\right)\right\}_{\lambda \in \Lambda}$ for $(f, v)$ is said to be cyclic if $\Phi\left(A_{a}\right)=A_{b}, \Phi\left(B_{a}\right)=B_{b}$. A cyclic ranging system is said to be of finite type if $H_{*}\left(V_{0} \backslash B_{a}, A_{a}\right)$ is finitely generated.

These techniques will be applied to the case of circle-valued Morse functions.

2.3. Equivariant ranging systems. Now we will apply the techniques of the previous section to investigation of the incidence coefficients in the Novikov complex. Let $f: M \rightarrow S^{1}$ be a Morse function, and $v$ be an $f$ gradient. We will assume here that the class $[f] \in H^{1}(M, \mathbb{Z})$ is indivisible, so that for all $z \in \bar{M}$ we have that

$$
F(z)-F(z t)=1 .
$$

We assume also $S(f) \neq \varnothing$, and that 0 is a regular value of $F$. Choose a Riemannian metric on $M$. Then $\bar{M}$ obtains a $t$-invariant Riemannian metric.

Definition 2.3.1. Let $u$ be any $f$-gradient. Let $\Sigma$ be a non empty set of regular values of $F$ satisfying the following collection $(\mathcal{S})$ of consitions:

$(\mathcal{S}): \bullet$ the regular value 0 is in $\Sigma$;

- for every $A, B \in \mathbb{R}$ the set $\Sigma \cap F^{-1}([A, B])$ is finite;

- if $\sigma \in \Sigma$ then $\sigma+n \in \Sigma$ for all $n \in \mathbb{Z}$;

- if $\lambda, \mu \in \Sigma$ are adjacent, then there is only one critical value of $F$ between $\lambda$ and $\mu$.

A set $\left\{\left(A_{\sigma}, B_{\sigma}\right)\right\}_{\sigma \in \Sigma}$ is called tequivariant ranging system for $(F, u)$, if

(1) For every $\mu, \nu \in \Sigma, \mu<\nu$ we have: $\left\{\left(A_{\sigma}, B_{\sigma}\right)\right\}_{\sigma \in \Sigma, \mu \leqslant \sigma \leqslant \nu}$ is a ranging system for $\left(\left.F\right|_{\left.F^{-1}([\mu, \nu]), u\right)}\right.$.

(2) $A_{\sigma-n}=A_{\sigma} \cdot t^{n}$ and $B_{\sigma-n}=B_{\sigma} \cdot t^{n}$ for every $n \in \mathbb{Z}$.

Any cyclic ranging system on the cobordism $W=F^{-1}([0,1])$ determines in an obvious way a $t$-equivariant ranging system. Let $p$ and $q$ be critical points of $f$, such that ind $p=\operatorname{ind} q+1$. Let $l=$ ind $q$. Let

$$
V_{1}=F^{-1}(1), \quad V_{0}=F^{-1}(0), \quad W=F^{-1}([0,1]) .
$$

Assume that the ranging system is adapted to the pair $(p, q)$. We lift $p$ and $q$ to $\bar{M}$ in such a way that $\bar{q} \in t W$ and $\bar{p} \in W$. Put

$$
[[p]]=H_{[\mu(p), 0]}\left(\left[S_{-}(p)\right]\right) \in H_{l}\left(V_{0} \backslash B_{0}, A_{0}\right) .
$$


The cohomology class

$$
]] S_{+}(\bar{q}, v)\left[\left[\in H^{l}\left(V_{0} \backslash B_{0}, A_{0}\right)\right.\right.
$$

will be denoted by $]] q[[$. Put

$$
h=t^{-1} \circ H_{[0,-1]}: H^{l}\left(V_{0} \backslash B_{0}, A_{0}\right) \rightarrow H^{l}\left(V_{0} \backslash B_{0}, A_{0}\right) .
$$

The next three assertions form the first basic ingredient of the proof of the main theorem of [15] (see [15, Proposition 4.18] for the proof).

Proposition 2.3.2. We have

$$
n_{k}(\bar{p}, \bar{q} ; v)= \begin{cases}\langle]] q\left[\left[,(h(v))^{k}[[p]]\right\rangle\right. & \text { if } k \geqslant 0, \\ 0 & \text { if } k<0 .\end{cases}
$$

Lemma 2.3.3. Let $G$ be a finitely generated abelian group, $A$ an endomorphism of $G$, and $\lambda: G \rightarrow \mathbb{Z}$ a homomorphism. Then for every $p \in G$ the series

$$
\sum_{k \geqslant 0} \lambda\left(A^{k} p\right) t^{k} \in \mathbb{Z}[[t]]
$$

is a rational function of $t$ of the form $\frac{P(t)}{Q(t)}$, where $P, Q$ are polynomials and $Q(0)=1$.

Theorem 2.3.4. Let $f: M \rightarrow S^{1}$ be a Morse function, and $v$ be a transverse $f$-gradient. Let also $F: \bar{M} \rightarrow \mathbb{R}$ be a lift of $f$ and $p, q$ be critical points of $f$ such that ind $p=$ ind $q+1$.

(1) Assume that there exists a cyclic ranging system on the cobordism

$$
W=F^{-1}([0,1]),
$$

adapted to $p, q$. Then the incidence coefficient $N(p, q, v)$ is a rational function of the form $\frac{P(t)}{Q(t)}$, where $P, Q$ are polynomials and $Q(0)=1$.

(2) The set of transverse $f$-gradients admitting a cyclic ranging system adapted to $p, q$ is $C^{0}$-open in the set of all transverse $f$-gradients.

2.4. Almost transverse gradients. In this subsection we gathered some preliminaries necessary for construction of ranging systems for $C^{0}$-generic flows; this construction will be recalled in the following subsections.

Definition 2.4.1. Let $f: W \rightarrow[a, b]$ be a Morse function and $v$ an $f$ gradient. We say that a Morse function $\phi: W \rightarrow \mathbb{R}$ is adjusted to the pair $(f, v)$ if

(1) $S(\phi)=S(f)$

(2) $v$ is also a $\phi$-gradient, 
(3) the function $\phi-f$ is constant in a neighborhood of $V_{1}$, in a neighborhood of $V_{0}$, and in a neighborhood of each critical point of $f$.

Observe that it is not clear whether there is a ranging system for every gradient. It turns out that ranging systems exist for $C^{0}$-generic gradients. We outline the proof in the next subsections.

Definition 2.4.2. Let $f: W \rightarrow[a, b]$ be a Morse function on a compact cobordism $W$, and $v$ be an $f$-gradient. We say that $v$ satisfies the almost transversality assumption if for every pair $x, y$ of critical points of $f$ we have

$$
\text { (ind } x \leqslant \text { ind } y) \Rightarrow(D(x, v) \pitchfork D(y,-v)) \text {. }
$$

or equivalently

$$
\text { (ind } x \leqslant \text { ind } y) \Rightarrow(D(x, v) \cap D(y,-v)=\varnothing) .
$$

We say aslo that $v$ is almost good or almost transverse.

Lemma 2.4.3. If $V$ is almost good, then $K(\mathrm{ind} \leqslant s ; v)$ is compact for every $s$.

Definition 2.4.4. We say that a $f$-gradient is $\delta$-separated if there is an ordered Morse function $\phi: W \rightarrow[a, b]$, adjusted to $(f, v)$, with an ordering sequence $a_{0}, \ldots, a_{n+1}$, such that

(1) for every $p \in S(f)$ we have $D_{\delta}(p) \subset \phi^{-1}\left(\left(a_{k}, a_{k+1}\right)\right)$, where $k=\operatorname{ind} p$.

(2) for every $l: 0 \leqslant l \leqslant n$ and every $q \in S(f)$ with ind $q=l$ there is a Morse function

$$
\psi: W_{l} \rightarrow\left[a_{l}, a_{l+1}\right],
$$

adjusted to $\left(\left.\phi\right|_{W_{l}},\left.v\right|_{W_{l}}\right)$, where $W_{l}=\phi^{-1}\left(\left[a_{l}, a_{l+1}\right]\right)$, and a regular value $\mu \in\left(a_{l}, a_{l+1}\right)$ of $\psi$, such that

- $D_{\delta}(q) \subset \phi^{-1}\left(\left(a_{l}, \mu\right)\right)$,

- and for every $r \in S(f)$, ind $r=l, r \neq q$ we have

$$
D_{\delta}(r) \subset \phi^{-1}\left(\left(\mu, a_{l+1}\right)\right) \text {. }
$$

Lemma 2.4.5. If $v$ is almost good, then $v$ is $\delta$-separated for some $\delta>0$.

Proposition 2.4.6. If $v$ is $\delta_{0}$-separated, then for every $\delta \in\left(0, \delta_{0}\right)$ and for every $s$ the set $D_{\delta}(\mathrm{ind} \leqslant s ; v)$ is compact.

Definition 2.4.7. A pair $(\phi, u)$ where $\phi$ is a Morse function on a cobordism and $u$ is a $\delta$-separated $\phi$-gradient will be called $\mathcal{A M}$-flow ${ }^{3}$.

\footnotetext{
${ }^{3}$ Usually the number $\delta$ is clear from the context, so we omit it from the notation.
} 
For an $\mathcal{A M}$-flow $(\phi, u)$ the sets $D_{\delta}($ ind $\leqslant s, u)$ form an increasing filtration of $W$. This is just one of versions of handle decomposition of $W$.

2.5. Stratified submanifolds and their thickenings. In this subsection we recall briefly the notions of $s$-submanifold and $t s$-submanifold introduced in [15]. These techniques will be used in the following subsection. We omit all proofs referring the reader to $[15, \S 2]$.

2.5.A. Stratified submanifolds. Let $\mathbb{A}=\left\{A_{0}, \ldots, A_{k}\right\}$ be a finite sequence of subsets of a topological space $X$. For $0 \leqslant s \leqslant k$, we denote $A_{s}$ also by $\mathbb{A}_{(s)}$; the set $A_{0} \cup \cdots \cup A_{s}$ is denoted by $A_{\leqslant s}$ and also by $\mathbb{A}_{(\leqslant s)}$. We say that $\mathbb{A}$ is a compact family if $\mathbb{A}_{(\leqslant s)}$ is compact for every $s$ with $0 \leqslant s \leqslant k$.

Definition 2.5.1. (Stratified submanifolds) Let $M$ be a manifold without boundary. A finite sequence $\mathbb{X}=\left\{X_{0}, \ldots, X_{k}\right\}$ of subsets of $M$ is called an $s$-submanifold of $M$ ( $s$ for "stratified") if

(1) $X_{i} \cap X_{j}=\varnothing$ for $i \neq j$, and each $X_{i}$ is a submanifold of $M$ of dimension $i$ with trivial normal bundle;

(2) $\mathbb{X}$ is a compact family.

For an $s$-submanifold $\mathbb{X}=\left\{X_{1}, \ldots, X_{s}\right\}$, the largest $k$ such that $X_{k} \neq \varnothing$ is called the dimension of $\mathbb{X}$ and is denoted by $\operatorname{dim} \mathbb{X}$. For a diffeomorphism $\Phi: M \rightarrow N$ and an $s$-submanifold $\mathbb{X}$ of $M$, we denote by $\Phi(\mathbb{X})$ the $s$ submanifold of $N$ defined by $\Phi(\mathbb{X})_{(i)}=\Phi\left(\mathbb{X}_{(i)}\right)$.

If $V$ is a submanifold of $M$ and $\mathbb{X}$ is an $s$-submanifold of $M$, then we say that $V$ is transversal to $\mathbb{X}$ (in symbols: $V \pitchfork \mathbb{X}$ ) if $V \pitchfork \mathbb{X}_{(i)}$ for each $i$. If $V$ is a compact submanifold of $M$ transversal to an $s$-submanifold $\mathbb{X}$, then the family $\left\{\mathbb{X}_{(i)} \cap V\right\}$ is an $s$-submanifold of $V$; it is denoted by $\mathbb{X} \cap V$.

Let $\mathbb{X}, \mathbb{Y}$ be two $s$-submanifolds of $M$. We say that $\mathbb{X}$ is transversal to $\mathbb{Y}$ (in symbols: $\mathbb{X} \pitchfork \mathbb{Y}$ ) if $\mathbb{X}_{(i)} \pitchfork \mathbb{Y}_{(j)}$ for every $i, j$. We say that $\mathbb{X}$ is almost transversal to $\mathbb{Y}$ (in symbols: $\mathbb{X} \nmid \mathbb{Y}$ ) if $\mathbb{X}_{(i)} \pitchfork \mathbb{Y}_{(j)}$ for any $i, j$ with $i+j<\operatorname{dim} M$. Note that $\mathbb{X} \nmid \mathbb{Y}$ if and only if $\mathbb{X}_{(\leqslant i)} \cap \mathbb{Y}_{(\leqslant j)}=\varnothing$ whenever $i+j<\operatorname{dim} M$.

Definition 2.5.2. Let $f: M \rightarrow \mathbb{R}$ be a Morse function, where $M$ is a closed manifold or a compact cobordism, and let $v$ be an $f$-gradient. Put

$$
\begin{aligned}
\mathbb{D}(v) & =\{D(\operatorname{ind} \leqslant i ; v)\}_{0 \leqslant i \leqslant \operatorname{dim} M}, \\
\mathbb{D}_{\lambda}(v) & =\left\{D(\operatorname{ind} \leqslant i ; v) \cap f^{-1}(\lambda)\right\}_{0 \leqslant i \leqslant \operatorname{dim} M} .
\end{aligned}
$$

Lemma 2.5.3. Assume that $v$ satisfies the almost transversality condition. Then:

$(1) \mathbb{D}(v)$ is a compact family; 
(2) if $M$ is a closed manifold, then $\mathbb{D}(v)$ is an s-submanifold transversal to $f^{-1}(\lambda)$ for every regular value $\lambda$ of $f$;

(3) if $M$ is a cobordism, then for every regular value $\lambda$ of $f$ the family $\mathbb{D}_{\lambda}(v)$ is an $s$-submanifold of $f^{-1}(\lambda)$.

2.5.B. Good fundamental systems of neighborhoods, and ts-submanifolds.

Definition 2.5.4. Let $X$ be a topological space, $\mathbb{A}=\left\{A_{0}, \ldots, A_{k}\right\}$ a compact family of subsets of $X, I$ an open interval $\left(0, \delta_{0}\right)$. A good fundamental system of neighborhoods of $\mathbb{A}$ (briefly: a $g f n$-system for $\mathbb{A}$ ) is a family $\mathbf{A}=\left\{A_{s}(\delta)\right\}_{\delta \in I, 0 \leqslant s \leqslant k}$ of open subsets of $X$ such that for any positive integer $s \leqslant k$ the following conditions are fulfilled:

(FS1) for every $\delta \in I$ we have $A_{s} \subset A_{s}(\delta)$;

$(\mathrm{FS} 2) \delta_{1}<\delta_{2} \Rightarrow A_{s}\left(\delta_{1}\right) \subset A_{s}\left(\delta_{2}\right)$;

(FS3) $\overline{A_{\leqslant s}(\delta)}=\bigcap_{\theta>\delta}\left(A_{\leqslant s}(\theta)\right)$ for every $\delta \in I$.

(FS4) we have $A_{\leqslant s}=\bigcap_{\theta>0}\left(A_{\leqslant s}(\theta)\right)$.

The interval $I$ is called the interval of subdefinition of the $g f n$-system and $\mathbb{A}$ is called the core of $\mathbf{A}$. We shall denote $A_{s}(\delta)$ also by $\mathbf{A}_{(s)}(\delta)$ and $A_{\leqslant i}(\delta)$ also by $\mathbf{A}_{(\leqslant i)}(\delta)$.

Let $M$ be a manifold without boundary, $\mathbb{X}$ an $s$-submanifold of $M$, and $\mathbf{X}$ a $g f n$-system for $\mathbb{X}$. We say that $\mathbf{X}$ is a ts-submanifold of $M$ with core $\mathbb{X}$. For a $t s$-submanifold $\mathbf{X}=\left\{X_{s}(\delta)\right\}_{\delta \in I, 0 \leqslant s \leqslant k}$, we shall also denote $X_{i}(\delta)$ by $\mathbf{X}_{(i)}(\delta)$, and $X_{\leqslant i}(\delta)$ by $\mathbf{X}_{(\leqslant i)}(\delta)$. The basic example of a $g f n$-system and a $t s$-manifold is given by the next lemma.

Lemma 2.5.5. Let $M$ be a closed Riemannian manifold or a Riemanian cobordism of dimension $n$. Let $f: M \rightarrow \mathbb{R}$ be a Morse function and $v a$ $\delta$-separated $f$-gradient. Then the family

$$
\mathbf{D}(v)=\left\{B_{\delta}(\text { ind }=s ; v)\right\}_{\delta \in] 0, \epsilon[}, 0 \leqslant s \leqslant n
$$

is a gfn-system for $\mathbb{D}(v)$. If $M$ is a closed manifold, this family is a tssubmanifold with core $\mathbb{D}(v)$.

Observe that there is no canonical way for choosing an interval of definition for this system. We shall say that $\lambda>0$ is in an interval of definition of $\mathbf{D}(v)$ if there is $\epsilon>\lambda$ such that $\left\{B_{\delta}(\text { ind }=s ; v)\right\}_{\delta \in] 0, \epsilon[, 0 \leqslant s \leqslant n}$ is a $g f n$-system. If $v$ is $\lambda$-separated, then $\lambda$ is in an interval of definition of $\mathbf{D}(v)$.

2.5.C. Tracks of subsets, s-submanifolds, and ts-submanifolds.

Definition 2.5.6. Let $f: W \rightarrow[a, b]$ be a Morse function on a compact Riemannian cobordism $W$, and $v$ be an $f$-gradient. Let $X \subset V_{1}$. The set 
$\{\gamma(x, t ;-v) \mid t \geqslant 0, x \in X\}$ is called the track of $X$ (with respect to $v$ ) and is denoted by $T(X, v)$.

Lemma 2.5.7. (1) If $X$ is compact, then $T(X, v) \cup D(v)$ is compact.

(2) If $X$ is compact, and every $(-v)$-trajectory starting at a point of $X$ reaches $V_{0}$, then $T(X, v)$ is compact.

(3) For any $X$ we have $\overline{T(X, v) \cup D(v)}=T(\bar{X}, v) \cup D(v)$.

(4) For any $X$ and any $\delta>0$ we have $\overline{T(X, v) \cup B_{\delta}(v)}=T(\bar{X}, v) \cup D_{\delta}(v)$.

Definition 2.5.8. Let $v$ be an $f$-gradient satisfying the almost transversality condition. Let $\mathbb{A}$ be an $s$-submanifold $\left\{A_{0}, \ldots, A_{k}\right\}$ of $V_{1}$ such that $\mathbb{A} \nmid \mathbb{D}_{b}(-v)$. By convention we write $A_{-1}=\varnothing$. Put

$$
T A_{i}(v)=T\left(A_{i-1}, v\right) \cup D(\text { ind }=i ; v) ;
$$

The family

$$
\mathbb{T}(\mathbb{A}, v)=\left\{T A_{i}(v)\right\}_{0 \leqslant i \leqslant k+1}
$$

will be called the track of $\mathbb{A}$, and the family

$$
(-v)_{[b, \lambda]}^{\sim}(\mathbb{A})=\left\{T A_{i+1}(v) \cap f^{-1}(\lambda)\right\}_{0 \leqslant i \leqslant k} .
$$

will be called the $(-v)^{m}$-image of $\mathbb{A}$. If the values $b, \lambda$ are clear from the context, we shall abbreviate $(-v)_{[b, \lambda]}^{\sim}(\mathbb{A})$ to $(-v)^{m}(\mathbb{A})$.

Lemma 2.5.9. (1) $\mathbb{T}(\mathbb{A}, v)$ and $(-v)_{[b, \lambda]}^{\varkappa}(\mathbb{A})$ are compact families.

(2) If $\lambda$ is a regular value of $f$, then $(-v)_{[b, \lambda]}^{\sim}(\mathbb{A})$ is an s-submanifold of $f^{-1}(\lambda)$.

Let us proceed to tracks of $t s$-submanifolds.

Definition 2.5.10. Let $\mathbf{A}=\left\{A_{s}(\delta)\right\}_{\delta \in\left(0, \delta_{0}\right), 0 \leqslant s \leqslant k}$ be a $t s$-submanifold of $V_{1}$ with core $\mathbb{A}$. Assume that $v$ is $\delta_{1}$-separated. For $0<\delta<\min \left(\delta_{0}, \delta_{1}\right)$ and $0 \leqslant s \leqslant k+1$, we put

$$
T A_{s}(\delta, v)=T\left(A_{s-1}(\delta), v\right) \cup B_{\delta}(\operatorname{ind}=s ; v)
$$

(by definition, $A_{-1}(\delta)=\varnothing$ ).

It may happen that in order to turn the family $\left\{T A_{s}(\delta, v)\right\}$ into a gfnsystem, it is necessary to reduce its initial interval of definition.

Definition 2.5.11. Let $I=(0, \mu)$, and let $Z=\{Z(\delta)\}_{\delta \in I}$ be a family of subsets of some space $X$. Let $0<\nu<\mu$. The family $\{Z(\delta)\}_{\delta \in(0, \nu)}$ will be called a restriction of $Z$ to the interval $(0, \nu)$. 
It is proved in [15, Lemma 2.11 and Proposition 2.12] that there is $\epsilon \in\left(0, \min \left(\delta_{0}, \delta_{1}\right)\right)$ such that the restriction of the family $T A_{s}(\delta, v)$ to the interval $(0, \epsilon)$ is a $g f n$-system with the core $\mathbb{T}(\mathbb{A}, v)$.

Definition 2.5.12. This $g f n$-system will be denoted by $\mathbf{T}(\mathbf{A}, v)$; we call it the track of $\mathbf{A}$.

Observe that there is no canonical choice of an interval of definition for this system.

2.6. Ranging pairs. In this subsection, $W$ is a Riemannian cobordism, $f: W \rightarrow[a, b]$ a Morse function, $v$ an $f$-gradient satisfying the almost transversality condition, $n=\operatorname{dim} W$.

Definition 2.6.1. Let $\mathcal{V}_{0}=\left(\phi_{0}, u_{0}, \mathcal{U}_{0}\right), \mathcal{V}_{1}=\left(\phi_{1}, u_{1}, \mathcal{U}_{1}\right)$ be $\mathcal{A M}$-flows on $V_{0}$ and $V_{1}$. We say that $\left(\mathcal{V}_{0}, \mathcal{V}_{1}\right)$ is a ranging pair for $(f, v)$ if $\mathbb{D}_{a}(v) \nmid \mathbb{D}\left(-u_{0}\right)$, $\mathbb{D}_{b}(-v) \nmid \mathbb{D}\left(u_{1}\right)$, and there is a number $\delta>0$ such that for any $0 \leqslant s \leqslant n-1$ the following conditions are fulfilled:

(RP1) $\delta$ is in intervals of definition of $\mathbf{T}\left(\mathbf{D}\left(u_{1}\right), v\right)$ and of $\mathbf{T}\left(\mathbf{D}\left(-u_{0}\right),-v\right)$;

(RP2) the gradients $v, u_{0}$, and $u_{1}$ are $\delta$-separated;

(RP3) $\overline{\mathbf{T}\left(\mathbf{D}\left(u_{1}\right), v\right)_{(\leqslant s+1)}(\delta)} \cap V_{0} \subset \mathbf{D}\left(u_{0}\right)_{(\leqslant s)}(\delta)$;

$(\mathrm{RP} 4) \overline{\mathbf{T}\left(\mathbf{D}\left(-u_{0}\right),-v\right)_{(\leqslant s+1)}(\delta)} \cap V_{1} \subset \mathbf{D}\left(-u_{1}\right)_{(\leqslant s)}(\delta)$.

We say that $v$ satisfies condition $(\mathrm{RP})$ if $(f, v)$ has a ranging pair.

The next theorem ([15, Theorem 4.3]) is one of the basic results of [15]. We will not comment on the proof (it occupies $\S 3$ and first 2 pages of $\S 4$ of [15]), since the techniques of the proof will not be used in the present paper.

Theorem 2.6.2. Let $\epsilon>0$. Then there is an $f$-gradient $w$ satisfying the almost transversality condition and a ranging pair $\left(\mathcal{V}_{0}, \mathcal{V}_{1}\right)$ for $(f, w)$ such that $\|w-v\| \leqslant \epsilon$. Moreover given a neighborhood $U$ of $\partial W$ we can assume that $\operatorname{supp}(w-v) \subset U \backslash \partial W$.

Assume now that $(f, v)$ has a ranging pair $\left(\mathcal{V}_{0}, \mathcal{V}_{1}\right)$. We will now show that this ranging pair generates a family of ranging systems for $(f, v)$. Moreover, for every $p, q \in S(f)$ with ind $p=$ ind $q+1$, in this family there is a ranging system adapted to $p, q$.

Let $\Lambda=\left\{\lambda_{0}, \ldots, \lambda_{k}\right\}$ be a set of regular values of $f$ such that

$$
\lambda_{i}<\lambda_{i+1}, \lambda_{0}=a, \lambda_{k}=b,
$$

and in each $\left[\lambda_{i}, \lambda_{i+1}\right]$ there is only one critical value of $f$. We choose some $\delta^{\prime}>\delta$ in the interval of definition of $\mathbf{T}\left(\mathbf{D}\left(u_{1}\right), v\right)$ and $\mathbf{T}\left(\mathbf{D}\left(-u_{0}\right),-v\right)$ such 
that for every $0 \leqslant s \leqslant n-1$ we have

$$
\begin{gathered}
\overline{\mathbf{T}\left(\mathbf{D}\left(u_{1}\right), v\right)_{(\leqslant s+1)}\left(\delta^{\prime}\right)} \cap V_{0} \subset \mathbf{D}\left(u_{0}\right)_{(\leqslant s)}(\delta), \\
\overline{\mathbf{T}\left(\mathbf{D}\left(-u_{0}\right),-v\right)_{(\leqslant s+1)}\left(\delta^{\prime}\right)} \cap V_{1} \subset \mathbf{D}\left(-u_{1}\right)_{(\leqslant s)}(\delta)
\end{gathered}
$$

(such a number $\delta^{\prime}$ exists because $\delta$ belongs to an interval of definition of $\mathbf{T}\left(\mathbf{D}\left(u_{1}\right), v\right)$ and of $\left.\mathbf{T}\left(\mathbf{D}\left(-u_{0}\right),-v\right)\right)$. Also, we require that the gradients $v$, $u_{0}, u_{1}$ be $\delta^{\prime}$-separated.

Now, for each integer $s$ with $0 \leqslant s \leqslant n$ we define compact subsets $A_{\lambda}^{(s)}$ and $B_{\lambda}^{(s)}$ of $f^{-1}(\lambda)$. We set $A_{\lambda}^{(0)}=B_{\lambda}^{(0)}=\varnothing$. Let

$$
\delta=\delta_{k}<\delta_{k-1}<\cdots<\delta_{1}<\delta_{0}=\delta^{\prime}
$$

be a sequence of real numbers, and let $1 \leqslant s \leqslant n$. We put

$$
\begin{aligned}
& A_{\lambda_{l}}^{(s)}=\overline{\mathbf{T}\left(\mathbf{D}\left(u_{1}\right), v\right)_{(\leqslant s)}\left(\delta_{l}\right)} \cap f^{-1}\left(\lambda_{l}\right) \quad \text { for } \quad 0<l \leqslant k, \\
& A_{a}^{(s)}=\overline{\mathbf{D}\left(u_{0}\right)_{(\leqslant s-1)}(\delta)}=D_{\delta}\left(\text { ind } \leqslant s-1 ; u_{0}\right) ; \\
& B_{\lambda_{l}}^{(s)}=\overline{\mathbf{T}\left(\mathbf{D}\left(-u_{0}\right),-v\right)_{(\leqslant s)}\left(\delta_{k-l}\right)} \cap f^{-1}\left(\lambda_{l}\right) \quad \text { for } \quad 0 \leqslant l<k, \\
& B_{b}^{(s)}=\overline{\mathbf{D}\left(-u_{1}\right)_{(\leqslant s-1)}(\delta)}=D_{\delta}\left(\text { ind } \leqslant s-1 ;-u_{1}\right) .
\end{aligned}
$$

Clearly, $A_{b}^{(s)}=D_{\delta}\left(\right.$ ind $\left.\leqslant s-1 ; u_{1}\right)$ and $B_{a}^{(s)}=D_{\delta}\left(\right.$ ind $\left.\leqslant s-1 ;-u_{0}\right)$.

The following lemma is proved in [15, Lemma 4.14].

Lemma 2.6.3. (1) $A_{\lambda}^{(r)} \cap B_{\lambda}^{(s)}=\varnothing$ if $r+s \leqslant n$.

(2) If $0 \leqslant s \leqslant n-1$, then $\left\{\left(A_{\lambda}^{(s)}, B_{\lambda}^{(n-s-1)}\right)\right\}_{\lambda \in \Lambda}$ is a ranging system for $(f, v)$ adapted to every pair $p, q$ of critical points of $f$ with ind $p=s+1$, ind $q=s$.

(3) For every $0 \leqslant s \leqslant n-1$ the pair

$$
\left(V_{1} \backslash B_{b}^{(n-s-1)}, A_{b}^{(s)}\right)
$$

is homotopy equivalent to a finite $C W$-pair that has cells of dimension s only.

In the case when $W$ is cyclic we can strengthen the results above so to construct cyclic ranging systems for $C^{0}$-generic gradients.

Definition 2.6.4. Let $f W \rightarrow[a, b]$ be a Morse function on a cyclic cobor$\operatorname{dism} W$, and let $v$ be an $f$-gradient satisfying the almost transversality condition. An $\mathcal{M}$-flow $\mathcal{V}_{0}=(f, v, \mathcal{U})$ on $V_{0}$ is called a ranging flow for $(f, v)$ if $\left(\mathcal{V}_{0}, \Phi\left(\mathcal{V}_{0}\right)\right)$ is a ranging pair for $(f, v)$. 
We say that $v$ satisfies condition (RF) if there is a ranging flow for $(f, v)$.

The proof of the following theorem is similar to the proof of Theorem 2.6.2.

Theorem 2.6.5. Let $f: W \rightarrow[a, b]$ be a Morse function on a cyclic cobordism $W$. Let $\epsilon>0$. There is an $f$-gradient $w$ satisfying the almost transversality condition and a ranging flow $\left(\mathcal{V}_{0}, \mathcal{V}_{1}\right)$ for $(f, w)$ such that Moreover, given a neighborhood $U$ of $\partial W$ we can assume that

$$
\operatorname{supp}(w-v) \subset U \backslash \partial W .
$$

Definition 2.6.6. A ranging system $\left\{\left(A_{\lambda}, B_{\lambda}\right)\right\}_{\lambda \in \Lambda}$ for $(f, v)$ is said to be cyclic if $\Phi\left(A_{a}\right)=A_{b}$ and $\Phi\left(B_{a}\right)=B_{b}$. A cyclic ranging system is said to be of finite type if $H_{*}\left(V_{0} \backslash B_{a}, A_{a}\right)$ is finitely generated.

Similarly to the above for every ranging flow and any pair $p, q$ of critical points of adjacent indices there is a finite type cyclic ranging system adapted to $p, q$. We arrive therefore at the following result which together with theorem 2.3.4 proves the Main Theorem of [15].

Theorem 2.6.7. Let $f: M \rightarrow S^{1}$ be a Morse function. Let also $p, q$ be critical points of $f$ such that ind $p=\operatorname{ind} q+1$. Then the set of transverse $f$-gradients admitting a cyclic ranging system adapted to $p, q$ is $C^{0}$-dense in the set of all transverse $f$-gradients.

\section{MORSE MAPS $M \rightarrow S^{1}$}

3.1. Algebraic preliminaries. We will work here with the terminology of $\S 1.3 .1$.

Definition 3.1.1. We say that an element $\varkappa \in \mathbb{Z}\left(\pi_{1} M\right)_{\xi}^{-}$is of type $(\mathcal{L})$, if there are $r, q \in G$, a natural number $m$, an $m \times m$-matrix $\mathcal{A}=\left(a_{i j}\right)_{1 \leqslant i, j \leqslant m}$ where $a_{i j} \in \mathbb{Z} G_{(-1)}$ and vectors $\left(X_{i}\right)_{1 \leqslant i \leqslant m},\left(Y_{i}\right)_{1 \leqslant i \leqslant m}, X_{i}, Y_{i} \in \mathbb{Z} H$, such that

$$
\varkappa=r\left(\sum_{s \geqslant 0} \sum_{\substack{1 \leqslant i \leqslant m \\ 1 \leqslant j \leqslant m}} Y_{i} a_{i j}^{(s)} X_{j}\right) q
$$

where $a_{i j}^{(s)}$ are the entries of $\mathcal{A}^{s}$.

The next lemma follows from the definition of the homomorphism $\ell$.

Lemma 3.1.2. The elements of type $(\mathcal{L})$ are contained in $\operatorname{Im} \ell$.

Proposition 3.1.3. The elements of type $(\mathcal{L})$ are of exponential growth. 
Proof. It suffices to prove that every matrix entry of the matrix series $u=\sum_{s \geqslant 0} A^{s}$, where $A=\left(a_{i j}\right)$ and $a_{i j} \in \mathbb{Z} G_{(-1)}$, is of exponential growth. For an $(m \times m)$-matrix $B=\left(b_{i j}\right)$ we denote by $\|B\|$ the number $\max _{i, j}\left\|b_{i j}\right\|$. It is easy to check that $\|B C\| \leqslant\|B\| \cdot\|C\| \cdot m$. Let $A$ be $(m \times m)$-matrix. Then $\left\|A^{s}\right\| \leqslant\|A\|^{s} \cdot m^{s-1} \leqslant\|A\|^{s} \cdot m^{s}$. Let $1 \leqslant i, j \leqslant m$. Write $u_{i j}=\sum n_{g} g$. Then for $k \geqslant 0$ we have $\sum_{\xi(g)=-k}\left|n_{g}\right| \leqslant(m \cdot\|A\|)^{k}$. If $m\|A\| \leqslant 1$ this gives $\sum_{\xi(g) \geqslant-k}\left|n_{g}\right| \leqslant k+1 \leqslant e^{k}$. If $m\|A\|>1$ we have

$$
\sum_{\xi(g) \geqslant-k}\left|n_{g}\right| \leqslant \frac{(m\|A\|)^{k}-1}{(m\|A\|)-1}<D(m\|A\|)^{k} .
$$

Therefore, in any case there are $c, d>0$ such that for $k<0, k \in \mathbb{Z}$ we have

$$
\sum_{\xi(g) \geqslant k}\left|n_{g}\right| \leqslant c \cdot d^{-k}
$$

For $k \geqslant 0$ it is true obviously and this implies that $u$ is of exponential growth.

3.2. Statement of Theorem 3.2.1. Theorem A follows immediately from the next theorem.

Theorem 3.2.1. In the set $\mathcal{G} t(f)$ there is a subset $\mathcal{G} t_{1}(f)$ with the following properties:

(1) $\mathcal{G} t_{1}(f)$ is open and dense in $\mathcal{G} t(f)$ with respect to $C^{0}$ topology.

(2) If $v \in \mathcal{G} t_{1}(f)$ then for every $x, y \in S(f)$ with ind $x=$ ind $y+1$ the incidence coefficient $\widetilde{n}(\widetilde{x}, \widetilde{y} ; v)$ is of type $(\mathcal{L})$.

(3) Let $v \in \mathcal{G} t_{1}(f)$. Let $U$ be a neighborhood of $S(f)$. Then for every $w \in \mathcal{G} t_{1}(f)$ such that $w=v$ in $U$ and $w$ is sufficiently close to $v$ in $C^{0}$ topology we have: $\widetilde{n}(\widetilde{x}, \widetilde{y} ; v)=\widetilde{n}(\widetilde{x}, \widetilde{y} ; w)$ for every $x, y \in S(f)$ such that ind $x=$ ind $y+1$.

3.3. Generalities on intersection indices. Let $M$ be a manifold without boundary, $\mathcal{Q}: \widehat{M} \rightarrow M$ be a regular covering (not necessarily connected) with structure group $H$. We say, that a submanifold $N$ of $M$ is liftedoriented (resp. lifted-cooriented) if a lift $\hat{i}: N \hookrightarrow \widehat{M}$ of the inclusion map $i: N \hookrightarrow M$ is fixed, and $N$ is oriented (resp. cooriented). We shall denote $\widehat{i}(N)$ by $\hat{N}$.

Let $X \subset M$ and let $N$ be a lifted-oriented submanifold of $M$ such that $N \backslash \operatorname{Int} X$ is compact. Then $\widehat{N} \backslash \operatorname{Int} \mathcal{Q}^{-1}(X)$ is compact, and the orientation 
class

$$
\mu_{N \backslash \operatorname{Int} \mathcal{Q}^{-1}(X)} \in H_{n}\left(N, N \cap \operatorname{Int} \mathcal{Q}^{-1}(X)\right)
$$

is defined, where $n=\operatorname{dim} N$ (see [7, Theorem A8]). The image of this class in $H_{n}\left(\widehat{M}, \mathcal{Q}^{-1}(X)\right)$ will be denoted by $[\widehat{N}]_{M, X}$ (or simply by $[\widehat{N}]$ if this does not lead to confusion).

Let $L$ be a compact lifted-cooriented submanifold without boundary of $M$. Then there is the coorientation class

$$
] \widehat{L}\left[\in H^{m-l}(\widehat{M}, \widehat{M} \backslash \widehat{L}),\right.
$$

where $l=\operatorname{dim} L, m=\operatorname{dim} M$.

Assume that $X \cap L=\varnothing, N \pitchfork L$ and $n+l=m$. Then $\hat{N}$ is transversal to $\widehat{L}$, the set $\widehat{N} \cap \widehat{L}$ is finite, and the intersection index $\widehat{N} \sharp \widehat{L} \in \mathbb{Z}$ is defined. Denote by $j$ the inclusion $\left(\widehat{M}, \mathcal{Q}^{-1}(X)\right) \hookrightarrow(\widehat{M}, \widehat{M} \backslash \widehat{L})$. The next lemma is standard.

Lemma 3.3.1. $\widehat{N} \sharp \widehat{L}=j^{*}(] \widehat{L}[)([\widehat{N}])$.

3.4. Lifts of ranging systems. Let $f: W \rightarrow[a, b]$ be a Morse function on a compact Riemannian cobordism, $f^{-1}(b)=V_{1}, f^{-1}(a)=V_{0}, v$ be an $f$-gradient. Let $\mathcal{Q}: \widehat{W} \rightarrow W$ be a regular covering with a structure group $H$. The lift of $v$ to $\widehat{W}$ will be denoted by $\widehat{v}$. If $x \in W$ and $\gamma(x, t ; v)$ is defined on $[0, a]$, and $\widehat{x} \in \mathcal{Q}^{-1}(x)$, then the lift to $\widehat{W}$ of $\gamma(x, \cdot ; v)$, starting at $\widehat{x}$ is the $\widehat{v}$-trajectory $\gamma(x, \cdot ; \widehat{v})$. It is easy to define with the help of this lifting procedure a diffeomorphism

$$
\widehat{v}^{m}: \mathcal{Q}^{-1}\left(V_{1} \backslash K(-v)\right) \rightarrow \mathcal{Q}^{-1}\left(V_{0} \backslash K(v)\right) .
$$

For $X \subset V_{1}$ we denote by abuse of notation $\hat{v}^{m \rightarrow}(X \backslash K(-v))$ by $\hat{v}^{m \rightarrow}(X)$.

Let $N$ be a oriented-lifted submanifold of $V_{1}$. Then it is easy to see that $v^{m \rightarrow}(N)$ is a oriented-lifted submanifold of $V_{0}$.

Proposition 3.4.1. Let $\left\{\left(A_{\lambda}, B_{\lambda}\right)\right\}_{\lambda \in \Lambda}$ be a ranging system for $(f, v)$. There is a homomorphism

$$
\hat{H}(v): H_{*}\left(\mathcal{Q}^{-1}\left(V_{1} \backslash B_{b}\right), \mathcal{Q}^{-1}\left(A_{b}\right)\right) \rightarrow H_{*}\left(\mathcal{Q}^{-1}\left(V_{0} \backslash B_{a}\right), \mathcal{Q}^{-1}\left(A_{a}\right)\right)
$$

of right $\mathbb{Z} H$-modules, such that:

(1) If $N$ is an oriented-lifted submanifold of $V_{1} \backslash B_{b}$, such that $N \backslash \operatorname{Int} A_{b}$ is compact, then

$$
\hat{H}(v)([\hat{N}])=\left[(\stackrel{m}{v}(N))^{\wedge}\right] .
$$

(2) There is an $\epsilon>0$ such that for every $f$-gradient wwith $\|w-v\|<\epsilon$ we have $\hat{H}(v)=\hat{H}(w)$. 
Proof. An easy induction argument shows that it is sufficient to prove the proposition in the case $\operatorname{card} \Lambda=1$. Split the set $S(F)$ of all critical points of $f$ in a disjoint union of two subsets $S 1(f)$ and $S 2(f)$ in such a way that

- for every $p \in S 1(f)$ the condition (RS2) i) of Definition 2.2.1 holds,

- for every $p \in S 2(f)$ the condition (RS2) ii) of Definition 2.2.1 holds.

Pick Morse functions $\phi_{1}, \phi_{2}: W \rightarrow[a, b]$, adjusted to $(f, v)$, such that there is a regular value $\mu_{1}$ of $\phi_{1}$ and a regular value $\mu_{2}$ of $\phi_{2}$ satisfying the following:

- for every $p \in S 1(f)$ we have: $\phi_{1}(p)<\mu_{1}$ and $\phi_{2}(p)>\mu_{2}$.

- for every $p \in S 2(f)$ we have: $\phi_{1}(p)>\mu_{1}$ and $\phi_{2}(p)<\mu_{2}$.

Let $\delta>0$. Put

$$
D 1_{\delta}(v)=V_{0} \cap \bigcup_{p \in S 1(f)} D_{\delta}(p, v), \quad D 1_{\delta}(-v)=V_{1} \cap \bigcup_{p \in S 1(f)} D_{\delta}(p,-v) .
$$

By a certain abuse of notation the intersection of $\cup_{p \in S 1(f)} D(p, v)$ with $V_{0}$ will be denoted by $D 1_{0}(v)$. Put

$$
\Delta(\delta,-v)=D 2_{\delta}(-v) \cup(-v)^{m}\left(B_{a}\right), \quad \nabla(\delta, v)=D 1_{\delta}(v) \cup \stackrel{m}{v}\left(A_{b}\right) .
$$

The similar notations like

$$
D 2_{\delta}(-v), \quad \Delta(0,-v), \quad D 2_{0}(v)
$$

etc. are now clear without special definition. For $\delta>0$ sufficiently small we have

$$
\begin{array}{lll}
\forall p \in S 1(f): D_{\delta}(p) \subset \phi_{1}^{-1}\left(\left(a, \mu_{1}\right)\right) & \text { and } & D_{\delta}(p) \subset \phi_{2}^{-1}\left(\left(\mu_{2}, b\right)\right) ; \\
\forall p \in S 2(f): D_{\delta}(p) \subset \phi_{1}^{-1}\left(\left(\mu_{2}, b\right)\right) & \text { and } & D_{\delta}(p) \subset \phi_{2}^{-1}\left(\left(a, \mu_{2}\right)\right) .
\end{array}
$$

It is easy to prove that for $\delta>0$ sufficiently small we have:

$$
\nabla(\delta, v) \subset \operatorname{Int} A_{a}, \quad \Delta(\delta,-v) \subset \operatorname{Int} B_{b}, \quad \Delta(\delta,-v) \cap D 1_{\delta}(-v)=\varnothing .
$$

Fix some $\delta>0$ satisfying (D1) and (D2).

Let $0 \leqslant \mu^{\prime}<\mu \leqslant \delta$ and $U$ be any subset of $V_{1}$ such that

$$
\Delta(0,-v) \subset U \subset B_{b} \text { and } U \cap D 1_{\delta}(-v)=\varnothing .
$$

(for example $U=\Delta(\delta,-v)$ will do). Consider the following sequence of homomorphisms

$$
\begin{gathered}
H_{*}\left(\mathcal{Q}^{-1}\left(V_{1} \backslash B_{b}\right), \mathcal{Q}^{-1}\left(A_{b}\right)\right) \stackrel{\hat{I}_{*}}{\longrightarrow} H_{*}\left(\mathcal{Q}^{-1}\left(V_{1} \backslash U\right),\right. \\
\left.\mathcal{Q}^{-1}\left(A_{b} \cup D 1_{\mu}(-v)\right)\right) \stackrel{\mathrm{Exc}^{-1}}{\longrightarrow} H_{*}\left(\mathcal{Q}^{-1}\left(V_{1} \backslash\left(U \cup D 1_{\mu^{\prime}}(-v)\right)\right),\right. \\
\mathcal{Q}^{-1}\left(\left(A_{b} \cup D 1_{\mu}(-v)\right) \backslash D 1_{\mu^{\prime}}(-v)\right) \stackrel{\hat{v}_{*}^{m}}{\longrightarrow} H_{*}\left(\mathcal{Q}^{-1}\left(V_{0} \backslash B_{a}\right), \mathcal{Q}^{-1}\left(A_{a}\right)\right) .
\end{gathered}
$$


Here $\hat{I}$ is the corresponding inclusion. Note that the last arrow is well defined since

$$
(-v)^{m}\left(B_{a}\right) \subset U \text { and } D_{0}(-v) \cap V_{1} \subset D 2_{0}(-v) \cup D 1_{\mu^{\prime}}(-v) .
$$

All the three arrows are homomorphisms of right $\mathbb{Z} H$-modules. (This is obvious for the first two arrows, as for the last just observe that $\widehat{v}^{m \rightarrow}$ commutes with the right action of $H$.)

The composition $\widehat{v}_{*}^{m} \circ \operatorname{Exc}^{-1} \circ \widehat{I}_{*}$ of this sequence will be denoted by $\hat{H}\left(v ; \mu^{\prime}, \mu ; \mathcal{U}\right)$. An argument similar to the one in the beginning of the page 999 of [15] shows that this homomorphism does not depend neither on the choices of $U, \mu^{\prime}, \mu, \delta$, nor on the choice of presentation

$$
S(f)=S 1(f) \sqcup S 2(f)
$$

(if there is more then one such presentation). Therefore this homomorphism is determined by $v$, the ranging system $\left\{\left(A_{\lambda}, B_{\lambda}\right)\right\}_{\lambda \in \Lambda}$ and the covering $\mathcal{Q}: \widehat{W} \rightarrow W$. We shall denote it by $\widehat{H}(v)$.

The proof of properties (1) and (2) of $\hat{H}(v)$ is similar of the proof of properties (1) and (2) of [15, Proposition 4.10] and will be omitted.

\subsection{Lifts of equivariant ranging systems and the proof of Theo-}

rem 3.2.1. We return here to the terminology of $\S 1.3 .1$. We begin by some algebraic preliminaries. Let $M, N$ be right $\mathbb{Z} H$-modules and $f: M \rightarrow N$ be a homomorphism of abelian groups. We say that $f$ is $\theta$-semilinear, if we have $f(x h)=f(x) \theta h \theta^{-1}$ for every $x \in M$. If $M, N$ are free finitely generated $\mathbb{Z} H$-modules with bases $\left(e_{j}\right),\left(d_{i}\right)$, one can associate to each $\theta$-semilinear homomorphism $f: M \rightarrow N$ a matrix $M(f)=\left(m_{i j}\right)$ by the following rule: $f\left(e_{j}\right)=\sum_{i} d_{i} m_{i j}$.

If $M$ is a right $\mathbb{Z} H$-module and $f: M \rightarrow \mathbb{Z}$ is a homomorphism of abelian groups, then we shall say that $f$ is of finite type, if for every $m \in M$ the set of $h \in H$, such that $f(m h) \neq 0$ is finite. If $f: M \rightarrow \mathbb{Z}$ is a homomorphism of a finite type, then we define a homomorphism $\tilde{f}: M \rightarrow \mathbb{Z} H$ of $\mathbb{Z} H$-modules by $\tilde{f}(m)=\sum_{h \in H} f(m h) h^{-1}$.

Returning to Morse maps, let us assume that $f: M \rightarrow S^{1}$ belongs to an indivisible cohomology class in $H^{1}(M, \mathbb{Z})$. Further, denote by $\pi: \bar{M} \rightarrow M$ the (unique) infinite cyclic covering, such that $f \circ \pi \sim 0$. The universal covering $p: \widetilde{M} \rightarrow M$ factors as $p=\pi \circ \mathcal{Q}$ where $\mathcal{Q}: \widetilde{M} \rightarrow \bar{M}$ is a covering with structure group $H=\operatorname{Ker} \xi$. Let $u$ be an $f$-gradient, its lift to $\bar{M}$ will be denoted by the same letter $u$. Let $\left\{\left(A_{\sigma}, B_{\sigma}\right)\right\}_{\sigma \in \Sigma}$ be a $t$-equivariant ranging system for $(F, u)$. 
For $\nu, \mu \in \Sigma, \nu<\mu$ denote by $\widehat{H}_{[\mu, \nu]}(u)$ the homomorphism

$$
\widehat{H}\left(\left.u\right|_{F^{-1}([\nu, \mu])}\right) \text {, }
$$

associated by virtue of Proposition 3.4.1 to the ranging system

$$
\left\{\left(A_{\sigma}, B_{\sigma}\right)\right\}_{\sigma \in \Sigma, \nu \leqslant \sigma \leqslant \mu}
$$

and the covering $\mathcal{Q}$ (restricted to the cobordism $F^{-1}([\nu, \mu])$ ). Denote by $\widehat{H}_{[\mu, \mu]}(u)$ the identity homomorphism of

$$
H_{*}\left(\mathcal{Q}^{-1}\left(F^{-1}(\mu) \backslash B_{\mu}\right), \mathcal{Q}^{-1}\left(A_{\mu}\right)\right)
$$

to itself.

It follows from the construction that for every $g \in G$ with $\xi(g)=k \in \mathbb{Z}$ we have

$$
\widehat{H}_{[\mu+k, \nu+k]}(u)=R(g) \circ \widehat{H}_{[\mu, \nu]}(u) \circ R\left(g^{-1}\right) .
$$

We have also

$$
\widehat{H}_{[\nu, \theta]}(u) \circ \widehat{H}_{[\mu, \nu]}(u)=\widehat{H}_{[\mu, \theta]}(u)
$$

For $\nu \in \Sigma$ put

$$
\widehat{h}_{\nu}(u)=R\left(\theta^{-1}\right) \circ \widehat{H}_{[\nu, \nu-1]}(u) .
$$

Then $\widehat{h}_{\nu}(u)$ is a $\theta$-semilinear endomorphism of

$$
H_{*}\left(\mathcal{Q}^{-1}\left(F^{-1}(\nu) \backslash B_{\nu}\right), \mathcal{Q}^{-1}\left(A_{\nu}\right)\right) .
$$

We have obviously $\hat{H}_{[\nu, \nu-k]}(u)=R\left(\theta^{k}\right) \circ\left(\hat{h}_{\nu}(u)\right)^{k}$. The next lemma follows from Proposition 3.4.1.

Lemma 3.5.1. Let $\mu, \nu \in \Sigma, \nu \leqslant \mu$, and $k \in \mathbb{N}$. Let also $N$ be orientedlifted submanifold of $F^{-1}(\mu) \backslash B_{\mu}$ such that $N \backslash \operatorname{Int} A_{\mu}$ is compact, and $L$ be a cooriented-lifted compact submanifold of $F^{-1}(\nu) \backslash A_{\nu}$. Assume that $\operatorname{dim} N+\operatorname{dim} L=\operatorname{dim} M-1$. Then:

(1) $N_{k}^{\prime}=u_{[\mu, \nu-k]}^{\varkappa}(N)$ is an oriented-lifted submanifold of $F^{-1}(\nu-k) \backslash B_{\nu-k}$ such that $N_{k}^{\prime} \backslash \operatorname{Int} A_{\nu-k}$ is compact. If $N_{k}^{\prime} \pitchfork L t^{k}$, then $N_{k}^{\prime} \cap L t^{k}$ is finite and

$$
\widehat{N}_{k}^{\prime} \sharp \widehat{L} \theta^{k}=\widehat{i}^{*}(] \widehat{L}[)\left(\left(\widehat{h}_{\nu}(u)\right)^{k}\left(\left[\widehat{N}_{0}^{\prime}\right]\right)\right),
$$

where $\hat{i}$ is the inclusion map

$$
\left(\mathcal{Q}^{-1}\left(F^{-1}(\nu) \backslash B_{\nu}\right), \mathcal{Q}^{-1}\left(A_{\nu}\right)\right) \hookrightarrow \mathcal{Q}^{-1}\left(F^{-1}(\nu), \mathcal{Q}^{-1}\left(F^{-1}(\nu) \backslash \widehat{L}\right)\right) .
$$

(2) For every $f$-gradient $w$, sufficiently close to $u$ in $C^{0}$-topology, the family $\left\{\left(A_{\sigma}, B_{\sigma}\right)\right\}_{\sigma \in \Sigma}$ is also a t-equivariant ranging system for $(F, w)$ and $\widehat{h}_{\nu}(u)=\widehat{h}_{\nu}(w)$, and $\widehat{H}_{[\mu, \nu]}(u)=\widehat{H}_{[\mu, \nu]}(w)$. 
3.6. Proof of Theorem 3.2.1. It is easy to see that it suffices to prove our theorem for the case of indivisible homotopy class $[f] \in H^{1}(M, \mathbb{Z})$ and we make this assumption up to the end of this subsection.

Fix first two points $x, y \in S(f)$, ind $x=$ ind $y+1$. Recall that we have chosen a lift $\widetilde{x} \in \widetilde{M}$ for every $x \in S(f)$. Denote $\mathcal{Q}(\widetilde{x})$ by $\bar{x}$. We can assume that $F(\bar{y})<F(\bar{x}) \leqslant F(\bar{y})+1$. Denote $\operatorname{dim} M$ by $n$; denote ind $x$ by $l+1$, then ind $y=l$. Choose some set $\Sigma$ of regular values of $F$, satisfying $(\mathcal{S})$ of Definition 2.3.1.

Denote by $\theta$ the maximal element of $\Sigma$ with $\theta<F(\bar{x})$ and by $N(v)$ the intersection $D(\bar{x}, v) \cap F^{-1}(\theta)$. Let also $N(v)$ be an oriented submanifold of $F^{-1}(\theta)$ diffeomorphic to $S^{l}$. Denote by $\eta$ the minimal element of $\Sigma$, satisfying $\eta>F(\bar{y})$. Then $\eta \leqslant \theta<\eta+1$. Let $L(-v)$ be the intersection $D(\bar{y},-v) \cap F^{-1}(\eta)$. Then $L(-v)$ is a cooriented submanifold of $F^{-1}(\eta)$, diffeomorphic to $S^{n-1-l}$.

Denote by $W$ the cobordism $F^{-1}([\eta, \eta+1])$. Then $\bar{x} \in W^{\circ}$.

Denote $F^{-1}(\eta)$ by $V_{0}, F^{-1}(\eta+1)$ by $V_{1}$, and $\Sigma \cap[\eta, \eta+1]$ by $\Lambda$.

Let $\mathcal{G} t_{1}(f ; x, y)$ be the subset of $\mathcal{G} t(f)$, consisting of all the $f$-gradients $v$, such that there is an equivariant ranging system $\left\{\left(A_{\sigma}, B_{\sigma}\right)\right\}_{\sigma \in \Sigma}$ for $(F, v)$ satisfying

$$
\begin{gathered}
N(v) \cap B_{\theta}=\varnothing, \quad L(-v) \cap A_{\eta}=\varnothing, \\
\left\{\begin{array}{c}
\left(F^{-1}(\eta) \backslash B_{\eta}, A_{\eta}\right) \quad \text { has a homotopy type of a finite } \mathrm{CW} \text {-pair } \\
\text { having only } l \text {-dimensional cells. }
\end{array}\right.
\end{gathered}
$$

Now we shall prove three properties of the set $\mathcal{G} t_{1}(f ; x, y)$.

1) $\mathcal{G} t_{1}(f ; x, y)$ is an open and dense subset of $\mathcal{G} t(f)$ with respect to $C^{0}$ topology.

This can be proved in the same way as the open-and-dense property of $\mathcal{G} t_{0}(f ; x, y)$ in [15, Proposition 4.12 and Theorem 4.16].

2) If $v \in \mathcal{G} t_{1}(f ; x, y)$ then $\widetilde{n}(\widetilde{x}, \widetilde{y} ; v)$ satisfies the condition $(\mathcal{L})$.

The lifts $x \mapsto \widetilde{x}$ and $y \mapsto \widetilde{y}$ define a lift $\hat{N}(v)$ of $N(v)$ to $\mathcal{Q}^{-1}\left(F^{-1}(\theta)\right)$ and $\widehat{L}(-v)$ of $L(-v)$ to $\mathcal{Q}^{-1}\left(F^{-1}(\eta)\right)$. The $\mathbb{Z} H$-module

$$
\mathcal{H}=H_{l}\left(\mathcal{Q}^{-1}\left(F^{-1}(\eta) \backslash B_{\eta}\right), \mathcal{Q}^{-1}\left(A_{\eta}\right)\right)
$$

is free. Choose some basis $e_{1}, \ldots, e_{m}$ of this module. The homomorphism $\widehat{h}_{\eta}(v)$ of this module is $\theta$-semilinear. Denote by $\mathcal{B}=\left(b_{i j}\right)$ its matrix, and denote by $\mathcal{A}$ the matrix $\left(b_{i j} \theta\right)$. Let $a_{i j}^{(s)}$ be the coefficients of $\mathcal{A}^{s}$. Consider the element $\xi=\widehat{H}_{[\theta, \eta]}(v)([\hat{N}(v)])$ of $\mathcal{H}$; let $\xi=\sum e_{i} X_{i}$ with $X_{i} \in \mathbb{Z} H$. Consider $\beta=\hat{i}^{*}(] \widehat{L}(-v)[)$ as a homomorphism of $\mathcal{H}$ to $\mathbb{Z}$. It is of finite 
type. Denote $\tilde{\beta}\left(e_{j}\right)$ by $Y_{j}$. Then $Y_{j} \in \mathbb{Z} H$. We claim that

$$
\tilde{n}(\tilde{x}, \tilde{y} ; v)=\sum_{\substack{s \geqslant 0 \\ 1 \leqslant i \leqslant m, 1 \leqslant j \leqslant m}} Y_{i} \cdot a_{i j}^{(s)} \cdot X_{j}
$$

To prove it write

$$
\tilde{n}(\tilde{x}, \tilde{y} ; v)=\sum_{s \geqslant 0}\left(\sum_{h \in H} \nu\left(\tilde{x}, \tilde{y} h \theta^{s}\right) \cdot h \theta^{s}\right)
$$

(here $\nu\left(\widetilde{x}, \widetilde{y} h \theta^{s}\right)$ stands for the algebraic number of $(-v)$-trajectories, joining $\tilde{x}$ with $\tilde{y} h \theta^{s}$; note that since $F(\bar{x}) \leqslant F(\bar{y})+1$, there are no $(-v)$ trajectories joining $\widetilde{x}$ to $\widetilde{y} g$ if $\xi(g)>0$ ).

To make the following computation more easy to comprehend, we introduce the following terminology conventions (valid only here). The homomorphism $\widehat{h}_{\eta}(v): \mathcal{H} \rightarrow \mathcal{H}$ will be denoted by $\mu$. We identify the cohomology classes in

$$
H^{*}\left(\mathcal{Q}^{-1}\left(F^{-1}(\nu), \mathcal{Q}^{-1}\left(F^{-1}(\nu) \backslash \widehat{L}\right)\right)\right.
$$

with their images in $H^{*}\left(\mathcal{Q}^{-1}\left(F^{-1}(\nu) \backslash B_{\nu}\right), \mathcal{Q}^{-1}\left(A_{\nu}\right)\right)$ (thus suppressing $\hat{i}^{*}$ in the notation). We have:

$$
\tilde{n}(\widetilde{x}, \widetilde{y} ; v)=\sum_{s \geqslant 0}\left(\sum_{h \in H}(] \widehat{L} \cdot h\left[\left(\mu^{s}(\xi)\right)\right) h\right) \theta^{s}
$$

(by Lemma 3.5.1). The latter expression equals

$$
\begin{aligned}
\sum_{s \geqslant 0}\left(\sum_{h \in H}(] \widehat{L}\left[\left(\mu^{s}(\xi) \cdot h^{-1}\right)\right) h\right) \theta^{s}=\sum_{s \geqslant 0}\left(\sum_{h \in H} \beta\left(\mu^{s}(\xi) \cdot h^{-1}\right) h\right) \theta^{s} & = \\
& =\sum_{s \geqslant 0} \widetilde{\beta}\left(\mu^{s}(\xi)\right) \cdot \theta^{s} .
\end{aligned}
$$

To obtain from this expression the formula (3.3) we need only a lemma, allowing to calculate $\mu^{s}(\xi)$ in terms of the coordinates of $\xi$ and the matrix of $\mu$ (the expression differs slightly from the standard linear-algebraic one since $\mu$ is $\theta$-semilinear).

Lemma 3.6.1. Let $F$ be a free $\mathbb{Z} H$-module with a basis $e_{1}, \ldots, e_{m}$ and $\mu: F \rightarrow F$ be a $\theta$-semilinear homomorphism of $F$. Let $m_{i j}$ be its matrix. Denote by $M$ the $m \times m$ matrix $\left(m_{i j} \cdot \theta\right)$. Let also $\xi \in F$ and $\xi=\sum e_{j} \xi_{j}$. Then for every natural $s \geqslant 0$ we have

$$
\mu^{s}(\xi)=\sum_{i, j} e_{i}\left[M^{s}\right]_{i j} \xi_{j} \theta^{-s}
$$


Proof. We will use induction in $s$. We have

$$
\begin{aligned}
\mu^{s+1}(\xi) & =\mu\left(\mu^{s}(\xi)\right)=\mu\left(\sum_{i} e_{i}\left(\sum_{j}\left[M^{s}\right]_{i j} \xi_{j} \theta^{-s}\right)\right)= \\
& =\sum_{i} \mu\left(e_{i}\right) \cdot\left(\theta \sum_{j}\left[M^{s}\right]_{i j} \xi_{j} \theta^{-s-1}\right)= \\
& =\sum_{i}\left(\sum_{k} e_{k} m_{k i}\right) \cdot\left(\theta \sum_{j}\left[M^{s}\right]_{i j} \xi_{j} \theta^{-s-1}\right)= \\
& =\sum_{k} e_{k}\left(\sum_{i}\left(m_{k i} \theta\right) \cdot\left[M^{s}\right]_{i j}\right) \xi_{j} \theta^{-s-1} .
\end{aligned}
$$

Now substitute the expression for $\mu^{s}(\xi)$ into the above formula, and the proof of (3.3) is over.

3) Stability with respect to perturbations of the gradient.

Let $v \in \mathcal{G} t_{1}(f ; x, y)$ and $U$ be a neighborhood of $S(f)$. We are going to prove that there is $\epsilon>0$ such that for every $w \in \mathcal{G} t_{1}(f ; x, y)$ with $\|w-v\|<\epsilon$ and $\left.w\right|_{U}=\left.v\right|_{U}$ we have: $\widetilde{n}(\widetilde{x}, \widetilde{y} ; v)=\widetilde{n}(\widetilde{x}, \widetilde{y} ; w)$.

Let $w$ be an $f$-gradient, sufficiently close to $v$. Then $\left\{\left(A_{\sigma}, B_{\sigma}\right)\right\}_{\sigma \in \Sigma}$ is still a $t$-equivariant ranging system for $(F, w)$, satisfying the conditions $(3.1)$ and (3.2). It is not difficult to see that

$$
[\widehat{N}(w)]=[\widehat{N}(v)], \quad[\widehat{L}(-w)]=[\widehat{L}(-v)] .
$$

Then Lemma 3.5.1 together with the formula (3.3) completes the proof.

Define now the set $\mathcal{G} t_{1}(f)$ to be the intersection of all the sets $\mathcal{G} t_{1}(f ; x, y)$ where $x, y$ range over the critical points of $f$ such that ind $x=$ ind $y+1$, and Theorem 3.2.1 is proved.

Remark 3.6.2. There is an obvious analogue of Theorem 3.2.1 or any regular covering $\phi: \widehat{M} \rightarrow M$ such that $f \circ \phi$ is homotopic to zero.

\section{Morse FORMS Within ARBITRARY COHOMOLOGY CLASSES}

Let $\omega$ be a Morse form of irrationality degree $q, v$ be an $\omega$-gradient. Let $\mathcal{Q}: N \rightarrow M$ be the minimal free abelian covering such that $\mathcal{Q}^{*} \omega$ is an exact form, that is, $\mathcal{Q}^{*} \omega=d F$ where $F: N \rightarrow \mathbb{R}$ is a Morse function. The structure group of $\mathcal{Q}$ is isomorphic to $\mathbb{Z}^{m}$. If $m=1$, the level surfaces $F^{-1}(a)$ and the cobordisms $F^{-1}([a, b])$ are compact. If $m>1$ these manifolds are non-compact, and this is the main reason why the results of [15] do not carry over directly to this case $m>1$.

The way round this difficulty consists in approximating the form $\omega$ by a rational 1-form $\omega_{1}$, so that $\omega_{1}=d \phi$ where $\phi$ is a circle-valued Morse 
function. Then the gradient $v$ is approximated (in $C^{0}$-topology) by a vector field $w$ which is a gradient for both $\omega$ and $\phi$ and such that its incidence coefficients are rational functions.

This program is realized in the present section.

4.1. Algebraic preliminaries. We will need some lemmas about the ring $\mathbb{Z}\left[\mathbb{Z}^{m}\right]$ and its completions and localizations.

Definition 4.1.1. Let $\eta: \mathbb{Z}^{m} \rightarrow \mathbb{R}$ be a non-zero homomorphism. We extend it to a linear map $\mathbb{R}^{m} \rightarrow \mathbb{R}$, which will be denoted by the same letter. We say that a set $Z \subset \mathbb{R}^{m}$ is an $\eta$-cone, if there is a compact convex nonempty set $K \subset \eta^{-1}(-1)$ such that

$$
Z=\{\lambda z \mid \lambda \in \mathbb{R}, \lambda \geqslant 0, z \in K\} .
$$

We say that $Z$ is $(\xi, \eta)$-cone if $Z$ is $\xi$-cone and $\eta$-cone. We say that a set $Z \subset \mathbb{R}^{m}$ is an integral $\eta$-cone if there are $e_{1}, \ldots, e_{k} \in \mathbb{Z}^{m}$, such that

(1) $\operatorname{rk}\left(e_{1}, \ldots, e_{k}\right)=m$,

(2) $\eta\left(e_{i}\right)<0$,

(3) $Z=\left\{\lambda_{1} e_{1}+\ldots+\lambda_{k} e_{k} \mid \lambda_{i} \geqslant 0\right\}$.

We shall also write $Z=Z\left\langle e_{1}, \ldots, e_{k}\right\rangle$. We say, that $Z$ is an integral $(\xi, \eta)$ cone if the vectors $e_{1}, \ldots, e_{k}$ above satisfy $\xi\left(e_{i}\right)<0, \eta\left(e_{i}\right)<0$ for all $i$. Note that an integral $(\xi, \eta)$-cone is a $(\xi, \eta)$-cone.

Lemma 4.1.2. Let $Z$ be an $\left(\eta_{1}, \eta_{2}\right)$-cone. Then there is an integral $\left(\eta_{1}, \eta_{2}\right)$ cone $Z_{0} \supset Z$.

Proof. We assume that $\eta_{1}, \eta_{2}$ are linearly independent; the other case is considered similarly. Denote $\eta_{1}^{-1}(-1)$ by $H$, and the set $H \cap\left\{\eta_{2}(x)<0\right\}$ by $H_{0}$. Then $H_{0}$ is an open halfspace of $H$, containing $Z \cap H$. Denote by $\mathcal{L}$ the set $\left\{\lambda x \mid \lambda \geqslant 0, x \in \mathbb{Z}^{m}\right\}$, then $\mathcal{L}$ is everywhere dense in $H$ and in $H_{0}$. It is not difficult to prove that there is a finite subset $\mathcal{L}_{0} \subset \mathcal{L}$ such that $\mathcal{L}_{0} \subset H_{0}$ and $\left\langle\mathcal{L}_{0}\right\rangle \supset Z \cap H$. We can choose $\mathcal{L}_{0}$ so that $\operatorname{rk} \mathcal{L}_{0}=m$ and the lemma is proved.

Lemma 4.1.3. Let $\xi: \mathbb{R}^{m} \rightarrow \mathbb{R}$ be a non-zero linear form and $\epsilon>0$. Then there is a finite set I of linear forms $\theta_{i}: \mathbb{R}^{m} \rightarrow \mathbb{R}, i \in I$ with $\left\|\theta_{i}\right\|<\epsilon$ such that

(1) The set $\Gamma=\left\{x \in \mathbb{R}^{m} \mid\left(\xi+\theta_{i}\right)(x) \leqslant 0\right\}$ is a $\xi$-cone.

(2) There is an integral $\xi$-cone $\Gamma_{0}$ such that for every family $\left\{A_{i}\right\}_{i \in I}$ of real numbers there is $b \in \mathbb{Z}^{m}$ with the property:

$$
\left\{x \in \mathbb{R}^{m} \mid\left(\xi+\theta_{i}\right)(x) \leqslant A_{i}\right\} \subset \Gamma_{0}+b .
$$


Proof. (1) Pick any $m$ linearly independent linear forms

$$
\alpha_{1}, \ldots, \alpha_{m}: \mathbb{R}^{m} \rightarrow \mathbb{R}
$$

with $\left\|\alpha_{i}\right\|<\min (\epsilon,\|\xi\| / 2)$. I claim that the finite family

$$
\left\{\alpha_{1},-\alpha_{1}, \ldots, \alpha_{m},-\alpha_{m}\right\}
$$

of linear forms satisfy the conclusions of the Lemma. Note first that

$$
Z=\xi^{-1}(-1) \cap\left\{x \mid \forall i:\left(\xi+\alpha_{i}\right)(x) \leqslant 0,\left(\xi-\alpha_{i}\right)(x) \leqslant 0\right\}
$$

is non empty and compact. (Indeed, let $x$ be a vector such that $|x|=1$ and $\xi(x)=\|\xi\|$. Then $a=-\frac{x}{\xi(x)} \in Z$. Further, if $Z$ is not bounded, then there is a sequence $x_{n} \in Z$ such that $\left|x_{n}\right| \rightarrow \infty$. Consider the sequence $x_{n} /\left|x_{n}\right|$. We can assume that it converges to some $v$ with $\|v\|=1$. Since $\xi\left(x_{n}\right)=-1$, we have $\xi(v)=0$. Further, for every $i$ we have

$$
\left(\xi+\alpha_{i}\right)(v) \leqslant 0, \quad\left(\xi-\alpha_{i}\right)(v) \leqslant 0,
$$

therefore $\alpha_{i}(v)=0$ and $v=0$.) Further, $\Gamma \subset \xi^{-1}((-\infty, 0])$ and the intersection $\Gamma \cap \xi^{-1}(0)$ consists of 0 . Therefore $x \in \Gamma$ and $x \neq 0$ implies $x=\lambda y, y \in Z$.

(2) Choose a vector $x_{0} \in \mathbb{Z}^{m}$ such that $\xi\left(x_{0}\right)<0$ and for every $1 \leqslant i \leqslant m$ we have

$$
\left(\xi+\alpha_{i}\right)\left(x_{0}\right)<0, \quad\left(\xi-\alpha_{i}\right)\left(x_{0}\right)<0
$$

(such a vector exists; it suffices to note that $\left(\xi \pm \alpha_{i}\right)(a)=-1 \mp \frac{\alpha_{i}(x)}{\xi(x)}<0$, and to approximate $a$ by an element $\left.y_{0} \in \mathbf{Q}^{m}\right)$.

Denote $\left|\left(\xi \pm \alpha_{i}\right)\left(x_{0}\right)\right|$ by $\beta_{i}^{ \pm}>0$. Now $\left(\xi \pm \alpha_{i}\right)(x) \leqslant A_{i}$ implies

$$
\left(\xi \pm \alpha_{i}\right)\left(x+p x_{0}\right) \leqslant A_{i}-p \beta_{i}^{ \pm} .
$$

Therefore if $p$ is sufficiently big, we obtain (4.1) with $b=-p x_{0}$, or, equivalently, $\left\{x \in \mathbb{R}^{m} \mid\left(\xi+\theta_{i}\right)(x) \leqslant A_{i}\right\} \subset \Gamma+b$. Then apply Lemma 4.1.2 to obtain an integral $\xi$-cone $\Gamma_{0}$, such that $\Gamma \subset \Gamma_{0}$.

We will use some terminology and results from [11]. Let $\eta: \mathbb{Z}^{m} \rightarrow \mathbb{R}$ be a homomorphism and $Z=Z\left\langle e_{1}, \ldots, e_{k}\right\rangle$ be an integral $\xi$-cone. Denote $\mathbb{Z}^{m} \cap Z$ by $[Z]$, and the set $\left\{n_{1} e_{1}+\ldots+n_{k} e_{k} \mid n_{i} \in \mathbb{N}\right\}$ by $[\widetilde{Z}]$. Note that $[Z]$ and $[\widetilde{Z}]$ are submonoids of $\mathbb{Z}^{m},[\widetilde{Z}] \subset[Z]$, and $[Z]$ is finitely generated over $[\widetilde{Z}]$. Consider the group rings $\mathbb{Z}[Z]$ and $\mathbb{Z}[\widetilde{Z}]$ of the monoids $Z$ and $\widetilde{Z}$; since $\mathbb{Z}[\widetilde{Z}]$ is an image of the ring $\mathbb{Z}\left[t_{1}, \ldots, t_{k}\right]$, it is noetherian. The ring $\mathbb{Z}[Z]$ is finitely generated as a left module over $\mathbb{Z}[\widetilde{Z}]$, therefore it is also noetherian. 
Consider the submonoid $\{x \in Z \mid x \neq 0\}$ of $Z$ and denote its group ring by $\mathfrak{m}_{Z}$. It is an ideal of $\mathbb{Z}[Z]$. Denote by $\mathbb{Z}[Z]^{\wedge}$ the $\mathfrak{m}_{Z}$-completion of $\mathbb{Z}[Z]$, and by $S_{Z}$ the multiplicative set $1+\mathfrak{m}_{Z} \subset \mathbb{Z}[Z]$. Then

$$
\left(S_{Z}^{-1} \mathbb{Z}[Z]\right)^{\wedge}=(\mathbb{Z}[Z])^{\wedge}
$$

(see $\left[2\right.$, Ch. $3, \S 3$, Prop. 12]). The ring $(\mathbb{Z}[Z])^{\wedge}$ is easily identified with the ring of all the elements $\lambda \in(\mathbb{Z}[Z])^{\wedge}$ such that $\operatorname{supp} \lambda \subset Z$ (this latter ring is obviously a subring of $\left.\mathbb{Z}[Z]_{\eta}^{-}\right)$. The ring $(\mathbb{Z}[Z])^{\wedge}$ is faithfully flat over $S_{Z}^{-1} \mathbb{Z}[Z]$ (ibid. Prop. 9). Therefore, if $P, Q \in \mathbb{Z}[Z]$ and there is $x \in(\mathbb{Z}[Z])^{\wedge}$ such that $P=Q x$, then $x \in S_{Z}^{-1} \mathbb{Z}[Z]$ (ibid. Ch. $1, \S 3$ ).

Notice that $\sigma_{Z}:=\left\{t^{I} \mid I \in Z\right\}$ is a multiplicative subset of $\mathbb{Z}[Z]$. It is easy to see that $\sigma_{Z}^{-1} \mathbb{Z}[Z]=\mathbb{Z}\left[\mathbb{Z}^{m}\right]$ and

$$
\sigma_{Z}^{-1}\left(\mathbb{Z}[Z]^{\wedge}\right)=\left\{S \in(\mathbb{Z}[Z])^{\wedge} \mid \exists x \in \mathbb{Z}^{m}: \operatorname{supp} S \subset Z+x\right\} .
$$

The faithful flatness property cited above implies immediately that if $P, Q \in \mathbb{Z}\left[\mathbb{Z}^{m}\right]$ and there is $x \in \sigma_{Z}^{-1}\left(\mathbb{Z}[Z]^{\wedge}\right)$ such that $P=Q x$, then

$$
x \in \sigma_{Z}^{-1} S_{Z}^{-1} \mathbb{Z}[Z] .
$$

Let $\eta$ be a linear form. Put

$$
\mathfrak{s}_{\eta}=\left\{1+Q \mid \operatorname{supp} Q \subset \eta^{-1}((-\infty, 0))\right\},
$$

then $\mathfrak{s}_{\eta}$ is a multiplicative subset of $\mathbb{Z}\left[\mathbb{Z}^{m}\right]$.

Lemma 4.1.4. Let $\alpha: \mathbb{Z}^{m} \rightarrow \mathbb{Z}$ be an indivisible homomorphism. Denote Ker $\eta$ by $H$. Let $A$ be $(k \times k)$-matrix, such that $a_{i j} \in \mathbb{Z}\left[\mathbb{Z}^{m}\right]_{(-1)}$. Let $\xi=\left(\xi_{1}, \ldots, \xi_{k}\right), \eta=\left(\eta_{1}, \ldots, \eta_{k}\right)$ be vectors in $(\mathbb{Z} H)^{k}$. Denote by $a_{i j}^{(s)}$ the (ij)-coefficient of the matrix $A^{s}$. Then

$$
(1-\operatorname{det} A) \sum_{s \geqslant 0}\left(\sum_{i, j} \xi_{i} a_{i j}^{(s)} \eta_{j}\right) \in \mathbb{Z}\left[\mathbb{Z}^{m}\right] .
$$

Proof. It suffices to prove that every coefficient of the $(k \times k)$-matrix

$$
(1-\operatorname{det} A)\left(\sum_{s \geqslant 0} A^{s}\right)
$$

belongs to $\mathbb{Z}\left[\mathbb{Z}^{m}\right]$. Consider the matrix $1-A$. It is invertible in the ring $S_{\alpha}^{-1} \mathbb{Z}\left[\mathbb{Z}^{m}\right]$ and the Cramer rules imply $(1-\operatorname{det} A)(1-A)^{-1} \in \operatorname{Mat}\left(\mathbb{Z}\left[\mathbb{Z}^{m}\right]\right)$. On the other hand $(1-A)^{-1}=\sum_{s \geqslant 0} A^{s}$. 
4.2. Preliminaries on Morse forms and their gradients. In this subsection we will work with the terminology from 2.1. Let $\omega$ be a Morse form on a closed connected manifold $M$. Let $p: \widetilde{M} \rightarrow M$ be the universal covering and let $\widetilde{F}: \widetilde{M} \rightarrow \mathbb{R}$ be a Morse function such that $d \widetilde{F}=p^{*} \omega$. Note that $\widetilde{F}(x g)=\widetilde{F}(x)+\{\omega\}(g)$, where $g \in \pi_{1} M$. Choose a Riemannian metric on $M$. Then $\widetilde{M}$ obtains a $\pi_{1} M$-invariant Riemannian metric. If $v$ is an $\omega$-gradient we denote by the same letter $v$ its lifts to $\widetilde{M}$ and $\overline{\bar{M}}$, since there is no possibility of confusion. Assume that for every $x \in S(f)$ a lift $\widetilde{x}$ of $x$ to $\widetilde{M}$ is fixed. The length of a curve $\gamma$ will be denoted by $l(\gamma)$. The following simple and useful lemma is known since the early 80 s. I learned it from J.-Cl. Sikorav.

Lemma 4.2.1. Let $v$ be an $\omega$-gradient. Then there are constants $A, B>0$ such that for every $g \in \pi_{1} M$ and every $(-v)$-trajectory $\gamma$, joining $\widetilde{x}$ and $\widetilde{y} \cdot g$ we have:

$$
l(\gamma) \leqslant A-B\{\omega\}(g) .
$$

Proof. Choose any $\delta>0$ less than the injectivity radius of $M$ and less than $\min _{p, q \in S(\omega)} \rho(p, q)$. Then any non-contractible piecewise smooth loop in $M$ is longer than $\delta$. Therefore for any $x \in \widetilde{M}, 1 \neq g \in \pi_{1} M$, any piecewise smooth path in $\widetilde{M}$, joining a point of $D(x, \delta / 3)$ with a point of $D(x g, \delta / 3)$ has the length $\geqslant \delta / 3$. Also for $x, y \in \widetilde{M}$ with $p(x), p(y) \in S(\omega)$ any piecewise smooth path joining joining a point of $D(x, \delta / 3)$ with a point of $D(y, \delta / 3)$ has the length $\geqslant \frac{\delta}{3}$.

Let $^{4}$

$$
\left\{\Phi_{p}: U_{p} \rightarrow B^{n}(0, r)\right\}_{p \in S(\omega)}
$$

be an $\omega$-chart-system such that $\mathcal{G}\left(\bar{U}_{p}, \Phi_{p}\right) \leqslant C$ for every $p \in S(\omega)$ and that $r C<\frac{\delta}{12}$. This condition implies in particular that $U_{p} \subset D\left(p, \frac{\delta}{12}\right)$. Choose some lifts $\widetilde{U}_{p}$ of neighborhoods $U_{p}$, extending $x \mapsto \widetilde{x}$. Let $D>0$ be less than $\min _{x \notin \cup U_{p}} \omega(v(x))$. Denote $\|v\|$ by $E$.

Now let $\gamma$ be a $v$-trajectory, joining $\widetilde{x}$ with $\widetilde{y} g$, and let

$$
A_{0}=\widetilde{x}, A_{1}, \ldots, A_{n}, \widetilde{y}=A_{n+1}
$$

be the points in $p^{-1}(S(\omega))$ such that $\gamma$ intersects $\widetilde{U}_{A_{i}}$. Then

$$
(n+1) \delta / 3 \leqslant l(\gamma) .
$$

\footnotetext{
${ }^{4}$ Recall from [15, p. 975] that for a chart $\Phi: U \rightarrow V \subset \mathbb{R}^{n}$ of $M$ and $x \in U$ we denote by $\mathcal{G}(x, \Phi)$ the number $\sup _{x \in M, h \in T_{x} M, h \neq 0}\left(\max \left(|h|_{\rho} /\left|\Phi_{*} h\right|_{e},\left|\Phi_{*} h\right|_{e} /|h|_{\rho}\right)\right)$, where $\rho$ stands for the metric on $M$ and $e$ for the euclidean metric in $\mathbb{R}^{n}$.
} 
The length of the part of $\gamma$ inside of $\bigcup_{i=0}^{n+1} \widetilde{U}_{A_{i}}$ is not more than $2 r C(n+1)$. Denote by $t_{i}$ and $\tau_{i}$ the moments when $\gamma$ enters and leaves $\tilde{U}_{A_{i}}$. We have

$$
\int_{\tau_{i}}^{t_{i+1}} \omega(\dot{\gamma}) d t=-\int_{\tau_{i}}^{t_{i+1}} \omega(v) d t=\widetilde{F}\left(\gamma\left(t_{i+1}\right)\right)-\widetilde{F}\left(\gamma\left(\tau_{i}\right)\right)
$$

Therefore the total time which $\gamma$ can spend outside $\bigcup_{p} \widetilde{U}_{A_{i}}$ is not more than $|\widetilde{F}(\widetilde{y} g)-\widetilde{F}(\widetilde{x})| / D$, and the length of the corresponding part of the curve is at most $\frac{E}{D}|\widetilde{F}(\widetilde{y} g)-\widetilde{F}(\widetilde{x})|$. Since $\gamma$ joins $\widetilde{x}$ with $\widetilde{y} g$, the last expression is at most

$$
\frac{E}{D}(\widetilde{F}(\widetilde{x})-\widetilde{F}(\widetilde{y})-\{\omega\}(g))
$$

Therefore

$$
\begin{aligned}
l(\gamma) \leqslant 2 r C(n+1)+\frac{E}{D}(\widetilde{F}(\widetilde{x})-\widetilde{F}(\widetilde{y}))- & \frac{E}{D}\{\omega\}(g) \leqslant \\
& \leqslant \frac{6 r C}{\delta} l(\gamma)+\frac{A}{2}-\frac{B}{2}\{\omega\}(g),
\end{aligned}
$$

where $A$ is chosen so that $A / 2 \geqslant|\widetilde{F}(\widetilde{x})-\widetilde{F}(\widetilde{y})|$ for every $x, y \in S(f)$ and $B=2 E / D$. The inequality $l(\gamma) \leqslant A-B\{\omega\}(g)$ follows.

Let $\omega$ be a Morse form, and $\left\{\Phi_{p}: U_{p} \rightarrow B^{n}\left(0, r_{p}\right)\right\}_{p \in S(\omega)}$ be an $\omega$-chartsystem. Choose a basis $a_{1}, \ldots, a_{m}$ in $H_{1}(M, \mathbb{Z}) /$ Tors.

Lemma 4.2.2. There exist closed 1 -forms $\lambda_{1}, \ldots, \lambda_{m}$ on $M$, such that

$$
\left\langle\left[\lambda_{i}\right], a_{j}\right\rangle=\delta_{i j}
$$

and $\operatorname{supp} \lambda_{i} \cap \bar{U}_{p}=\varnothing$ for every $i$ and every $p \in S(\omega)$.

Proof. Let $\theta$ be any closed 1-form. Let

$$
\left\{\widetilde{\Phi}_{p}: U_{p}^{\prime} \rightarrow B^{n}\left(0, r_{p}^{\prime}\right)\right\}_{p \in S(\omega)}
$$

be some standard extension of

$$
\left\{\Phi_{p}: U_{p} \rightarrow B^{n}\left(0, r_{p}\right)\right\}_{p \in S(\omega)}
$$

and let $\phi_{p}$ be a $C^{\infty}$ function which equals to 1 in a neighborhood of $\bar{U}_{p}$ and $\operatorname{supp} \phi_{p} \subset U_{p}^{\prime}$. Let $F_{p}$ be a function on $U_{p}^{\prime}$, such that $d F_{p}=\theta$. Consider the form $\theta^{\prime}=\theta-\sum d\left(\phi_{p} F_{p}\right)$ where the summation ranges over all zeros of $\omega$. Then $\left[\theta^{\prime}\right]=[\theta]$ and $\theta(x)=0$ in every $\bar{U}_{p}$. 
Choose and fix closed 1-forms $\lambda_{1}, \ldots, \lambda_{m}$ on $M$ satisfying the conclusions of the previous lemma. For $\boldsymbol{\mu}=\left(\mu_{1}, \ldots, \mu_{m}\right) \in \mathbb{R}^{m}$ denote by $\boldsymbol{\mu} \cdot \boldsymbol{\lambda}$ the 1 -form $\sum_{i=1}^{m} \mu_{i} \lambda_{i}$, and by $\omega_{\boldsymbol{\mu}}$ the 1 -form $\omega+\boldsymbol{\mu} \cdot \boldsymbol{\lambda}$. For $\epsilon>0$ set

$$
\Omega_{\epsilon}=\left\{\omega_{\boldsymbol{\mu}}|| \boldsymbol{\mu}\left|=\max _{i}\right| \mu_{i} \mid \leqslant \epsilon\right\}
$$

Definition 4.2.3. We shall say that $\omega_{\epsilon}$ is a Morse family, if for every $\vec{\epsilon}$ with $|\boldsymbol{\mu}| \leqslant \epsilon$ the form $\omega_{\boldsymbol{\mu}}$ is a Morse form and $S\left(\omega_{\boldsymbol{\mu}}\right)=S(\omega)$. Let $\omega_{\epsilon}$ be a Morse family, and $v$ be a vector field. We say, that $v$ is an $\omega_{\epsilon^{-}}$gradient, if $v$ is an $\omega_{\epsilon}$-gradient for each $\omega_{\boldsymbol{\mu}} \in \omega_{\epsilon}$.

Lemma 4.2.4. 1) There is $\epsilon>0$, such that $\omega_{\epsilon}$ is a Morse family.

2) Let $v$ be an $\omega$-gradient. Then there is $\epsilon>0$ such that $v$ is an $\omega_{\epsilon}$-gradient.

3) Let $v$ be an $\omega_{\epsilon}$-gradient. Then there is $\delta>0$ such that every $\omega$-gradient $u$ with $\|u-v\|<\delta$ is an $\omega_{\epsilon}$-gradient.

Proof. 1) Put

$$
\lambda=\sup _{\substack{1 \leqslant i \leqslant m \\ x \in M}}\left\|\lambda_{i}(x)\right\|, \quad \quad \eta=\min _{x \in M \backslash \cup_{p} U_{p}}\|\omega(x)\| .
$$

Then $\epsilon=\frac{\eta}{2 m \lambda}$ will do.

2) Put

$$
\bar{\lambda}=\sup _{\substack{1 \leqslant i \leqslant m \\ x \in M}}\left\|\lambda_{i}(v)(x)\right\|, \quad \bar{\eta}=\min _{x \in M \backslash \cup_{p} U_{p}} \omega(v)(x) .
$$

3) Denote by $Q$ the compact set $\left(M \backslash \cup_{p} U_{p}\right) \times[-\epsilon, \epsilon]^{m}$ and by $F: Q \rightarrow \mathbb{R}$ the map $F:\left(x, \mu_{1}, \ldots, \mu_{m}\right) \mapsto \omega_{\boldsymbol{\mu}}(v)(x)$. Since $\operatorname{Im} F \subset(0, \infty)$, there is $\beta>0$ such that $\operatorname{Im} F \subset[\beta, \infty)$. Let $u$ be any $\omega$-gradient such that

$$
\|u-v\| \cdot(m \lambda \epsilon+\|\omega\|) \leqslant \frac{\beta}{2} .
$$

We claim that $u$ is an $\omega_{\epsilon}$-gradient. Indeed, note first that $\omega_{\boldsymbol{\mu}}(u)(x)>0$ for any $x \in\left(\underset{p}{\cup} U_{p} \backslash S(\omega)\right)$ and any $|\boldsymbol{\mu}| \leqslant \epsilon$. Further, if $x \in\left(M \backslash \cup_{p} U_{p}\right)$, we have

$$
\begin{aligned}
\left|\omega_{\boldsymbol{\mu}}(u)(x)-\omega_{\boldsymbol{\mu}}(v)(x)\right| & =\left|\left(\omega+\sum_{i} \mu_{i} \lambda_{i}\right)(u-v)(x)\right| \leqslant \\
& \leqslant\|u-v\| \cdot(\|\omega\|+\epsilon \lambda m) \leqslant \frac{\beta}{2},
\end{aligned}
$$

therefore $\omega_{\boldsymbol{\mu}}(x)>0$. Finally, $u$ has a standard form with respect to some $\omega$-chart-system. A suitable restriction of this system will be an $\omega_{\boldsymbol{\mu}}$-chartsystem for any $\boldsymbol{\mu}$ with $|\boldsymbol{\mu}|<\epsilon$. 
Now we can define the incidence coefficients with respect to the universal cover. The preceding lemma implies that $v$ is an $\bar{\omega}$-gradient for some 1form $\bar{\omega}$ which cohomology class is rational. Therefore (see [12]) for every $g \in \pi_{1}(M)$ there is at most finite set of $(-v)$-trajectories joining $\widetilde{x}$ with $\widetilde{y} g$ if ind $x=$ ind $y+1$. Choose orientations of descending discs. For each such trajectory we denote by $\epsilon(\gamma)$ the sign of intersection of $D(\widetilde{x}, v)$ with $D(\tilde{y} g,-v)$ along $\gamma$.

Definition 4.2.5. The element $\sum_{\gamma} \epsilon(\gamma)$ is denoted by $\nu(\widetilde{x}, \widetilde{y} g)$ and we set 5

$$
\widetilde{n}(\widetilde{x}, \widetilde{y} ; v)=\sum_{g \in G} \nu(\widetilde{x}, \tilde{y} g) g \in\left(\mathbb{Z}\left[\pi_{1} M\right]\right) \widehat{\wedge}
$$

Lemma 4.2.6. $\tilde{n}(\widetilde{x}, \tilde{y} ; v) \in\left(\mathbb{Z}\left[\pi_{1} M\right]\right)_{\{\omega\}}^{-}$.

Proof. Recall that Novikov ring $\mathbb{Z}\left(\left[\pi_{1} M\right]\right)_{\{\omega\}}^{-}$consists of all $\lambda \in\left(\mathbb{Z}\left[\pi_{1} M\right]\right)^{\wedge}$ such that for every $c \in \mathbb{R}$ we have: $\operatorname{supp} \lambda \cap\{\omega\}^{-1}([c, \infty))$ is finite. Then our lemma follows from Lemma 4.2.1.

Remark 4.2.7. Note that the analogs of Lemmas 4.2.1 and 4.2.6 are obviously true for any regular covering $p^{\prime}: \widehat{M} \rightarrow M$ such that $\left(p^{\prime}\right)^{*}[\omega]=0$.

4.3. Incidence coefficients with respect to a free abelian cover. Next we pass to free abelian covers. Assume here the terminology of Subsection 1.3.2 of Introduction. The universal covering factors then as $p=\mathcal{P} \circ \mathcal{Q}$, where $\mathcal{Q}: \widetilde{M} \rightarrow \overline{\bar{M}}$. The epimorphism $\pi_{1}(M) \rightarrow H_{1}(M, \mathbb{Z}) /$ Tors will be denoted by $Q$. The de Rham cohomology class $[\omega]$ of $\omega$ defines a homomorphism $H^{1}(M, \mathbb{Z}) /$ Tors $\rightarrow \mathbb{R}$, which will be denoted by the same letter $[\omega]$. Note that $\{\omega\}=[\omega] \circ Q$. Since $\mathcal{P}^{*}\left(H^{1}(M, \mathbb{R})\right)=0$, there is a Morse function $\overline{\bar{F}}: \overline{\bar{M}} \rightarrow \mathbb{R}$, such that $d \overline{\bar{F}}=\mathcal{P}^{*} \omega$, and we shall assume that $\widetilde{F}=\overline{\bar{F}} \circ Q$.

We have chosen a Riemannian metric on $M$, whence the manifold $\overline{\bar{M}}$ obtains a Riemannian metric, which is $\mathbb{Z}^{m}$-invariant. We have also chosen a basis $\left(a_{1}, \ldots, a_{m}\right)$ in $H_{1}(M, \mathbb{Z}) /$ Tors. Therefore this group is identified with $\mathbb{Z}^{m}$, and the vector space $H^{1}(M, \mathbb{R})$ with the dual space of linear forms $\mathbb{R}^{m} \rightarrow \mathbb{R}$. Choose the $L_{1}$-norm in $\mathbb{R}^{m}$. Then the dual space obtains the sup-norm. (That is $\left\|\sum_{i} \alpha_{i} a_{i}\right\|=\sum_{i}\left|\alpha_{i}\right|$ and $\left\|\sum_{i} \beta_{i} a_{i}^{*}\right\|=\max _{j}\left|\beta_{j}\right|$, where $\left\{a_{i}^{*}\right\}$ is the base dual to $\left\{a_{i}\right\}$.)

\footnotetext{
${ }^{5}$ Recall that $\left(\mathbb{Z}\left[\pi_{1} M\right]\right)^{\wedge}$ is the abelian group of all the formal linear combinations (infinite in general) of the elements of $G$ with integer coefficients.
} 
Corollary 4.3.1. Let $v$ be an $\omega$-gradient. There is $\epsilon>0$, such that every linear form $\eta: \mathbb{R}^{m} \rightarrow \mathbb{R}$ with $\|[\omega]-\eta\| \leqslant \epsilon$ is a cohomology class of a Morse form $\omega(\eta)$ such that $v$ is an $\omega$-gradient.

Proof. Every linear form $\eta: \mathbb{R}^{m} \rightarrow \mathbb{R}$ with $\|[\omega]-\eta\| \leqslant \epsilon$ can be written as $\eta=[\omega]+\sum \eta_{i} a_{i}^{*}$ where $\eta_{i} \in \mathbb{R},\left|\eta_{i}\right| \leqslant \epsilon$. Now let $\epsilon>0$ be so small that $\omega_{\epsilon}$ is a Morse family and $v$ is an $\omega_{\epsilon}$-gradient and set $\omega(\eta)=\omega+\sum \eta_{i} \lambda_{i}$.

Definition 4.3.2. For two critical points $x, y \in S(\omega)$ and an $\omega$-gradient $v$ we set

$$
I(x, y ; v)=\left\{g \in \pi_{1}(M) \mid \text { there is a }(-v) \text {-trajectory } \gamma \text { joining } \tilde{x} \text { to } \tilde{y} \cdot g\right\} .
$$

If the set of $(-v)$-trajectories joining $\tilde{x}$ to $\tilde{y} \cdot g$ is finite, we denote by $N(\tilde{x}, \tilde{y}, g ; v)$ its cardinality. (We identify here two trajectories which differ by a parameter change.)

Remark 4.3.3. If $v$ is a good $\omega$-gradient and elements $x, y \in S(\omega)$ satisfy ind $x=\operatorname{ind} y+1$, then $\operatorname{supp}(\tilde{n}(\tilde{x}, \tilde{y} ; v)) \subset I(x, y ; v)$ and for every $g \in \pi_{1} M$ the set $N(\tilde{x}, \tilde{y}, g ; v)$ is finite.

Lemma 4.3.4. There is an integral $[\omega]$-cone $\Gamma$ and a vector $a \in \mathbb{Z}^{m}$ such that

$$
Q(I(x, y ; v)) \subset \Gamma+a .
$$

Proof. Note that if $v$ is a $\varkappa$-gradient for some Morse form $\varkappa$, then

$$
Q(I(x, y ; v)) \subset\left[\varkappa^{-1}\right]((-\infty, A])
$$

for some $A$. By 4.3.1 there is $\epsilon>0$, such that every linear form $\eta: \mathbb{R}^{m} \rightarrow \mathbb{R}$ with $\|[\omega]-\eta\| \leqslant \epsilon$ is the cohomology class of a Morse form $\omega(\eta)$ such that $v$ is an $\omega(\eta)$-gradient. Choose then the linear forms $\eta_{i}$ so as to satisfy Lemma 4.1.3 and obtain the conclusion of the Lemma.

Lemma 4.3.5. Let $\gamma, \gamma^{\prime}$ be two Morse forms with $[\gamma] \neq 0,\left[\gamma^{\prime}\right] \neq 0$. Assume that a vector field $v$ satisfying the transversality assumption is $\gamma$-gradient and $\gamma^{\prime}$-gradient. Let $x, y \in S(\gamma)$, ind $x=$ ind $y+1$. Then

1) If $[\gamma]=\alpha\left[\gamma^{\prime}\right]$ with $\alpha<0$, then $I(x, y ; v)$ is finite.

2) If there is no $\alpha<0$ with $[\gamma]=\alpha\left[\gamma^{\prime}\right]$, then there is an integral $\left([\gamma],\left[\gamma^{\prime}\right]\right)$ cone $\Delta$ and $b \in \mathbb{Z}^{m}$ such that $Q(I(x, y ; v)) \subset \Delta+b$.

Proof. 1) Obvious.

2) If $[\gamma]$ and $\left[\gamma^{\prime}\right]$ are linearly dependent, then $[\gamma]=\alpha\left[\gamma^{\prime}\right]$ with $\alpha>0$ and our lemma follows from Lemma 4.3.4. Therefore we can assume that $[\gamma]$ and $\left[\gamma^{\prime}\right]$ are linearly independent, which imply that there is $h \in \mathbb{Z}^{m}$ 
with $[\gamma](h),\left[\gamma^{\prime}\right](h)<0$. From Lemma 4.3 .4 we know that there are integral $[\gamma]$-cone $\Gamma_{1}$ and $a_{1} \in \mathbb{Z}^{m}$ such that $Q(I(x, y ; v)) \subset \Gamma_{1}+a_{1}$.

Also there are integral $\left[\gamma^{\prime}\right]$-cone $\Gamma_{2}$ and $a_{2} \in \mathbb{Z}^{m}$ such that such that $Q(I(x, y ; v)) \subset \Gamma_{2}+a_{2}$. Adding to the generators of $\Gamma_{1}$ and $\Gamma_{2}$ some integral vectors we can assume that $h \in \operatorname{Int} \Gamma_{1}, h \in \operatorname{Int} \Gamma_{2}$. Then there exists $N \in \mathbb{N}$ such that $\Gamma_{1}+a_{1} \subset \Gamma_{1}-N h$ and $\Gamma_{2}-N h \supset \Gamma_{2}+a_{2}$. Thus

$$
Q(I(x, y ; v)) \subset\left(\Gamma_{1}-N h\right) \cap\left(\Gamma_{2}-N h\right)=\Gamma_{1} \cap \Gamma_{2}-N h .
$$

The set $\Gamma_{1} \cap \Gamma_{2}$ is a $\left([\gamma],\left[\gamma^{\prime}\right]\right)$-cone, and by $(3.1)$ there is an integral $\left([\gamma],\left[\gamma^{\prime}\right]\right)$ cone $\Delta$ such that $\Gamma_{1} \cap \Gamma_{2} \subset \Delta$ therefore $Q(I(x, y ; v)) \subset \Delta-N h$.

By Remark 4.2.7 the incidence coefficient $\left.\overline{\bar{n}}(\overline{\bar{x}}, \overline{\bar{y}} ; v) \in\left(\mathbb{Z}^{m} \mathbb{Z}^{m}\right]\right)_{[\omega]}^{-}$is defined. We shall assume that the lifts $\overline{\bar{x}}$ of points $x \in S(\omega)$ are chosen so that $Q(\widetilde{x})=\overline{\bar{x}}$. Note that obviously $\operatorname{supp}(\overline{\bar{n}}(\overline{\bar{x}}, \overline{\bar{y}} ; v)) \subset Q(\operatorname{supp} \tilde{n}(\widetilde{x}, \widetilde{y} ; v))$.

4.4. Proof of Theorem B. For a Morse form $\xi$ such that $[\xi] \in H^{1}(M, \mathbf{Q})$, $[\xi] \neq 0$ we denote by $\xi_{0}$ the (unique) Morse form, such that $\left[\xi_{0}\right]$ is an indivisible class in $H^{1}(M, \mathbb{Z})$ and that $\xi_{0}=\mu \xi$ with $\mu>0$. The map $M \rightarrow S^{1}$, corresponding to $\xi_{0}$, will be denoted by $f_{0}\langle\xi\rangle$.

Definition 4.4.1. Define now $\mathcal{G} t_{1}(\omega)$ as the set of all $\omega$-gradients $v \in \mathcal{G} t(\omega)$, satisfying the following property:

$(\mathcal{C})$ There is $\epsilon: 0<\epsilon \leqslant\|[\omega]\| / 2$ such that $v$ is an $\omega_{\epsilon}$-gradient and there is a Morse form $\xi \in \omega_{\epsilon}$ with $[\xi] \in H^{1}(M, \mathbf{Q})$ such that $v \in \mathcal{G} t_{1}\left(f_{0}\langle\xi\rangle\right)$.

We shall now prove the properties of $\mathcal{G} t_{1}(\omega)$ required in the statement.

1) $\mathcal{G} t_{1}(\omega)$ is $C^{0}$-open in $\mathcal{G} t(\omega)$. Indeed, if $v$ satisfies $(\mathcal{C})$ then every $\omega$-gradient $u$, sufficiently close to $v$, is also an $\omega_{\epsilon}$-gradient (due to Lemma 4.2 .4 ) and $u \in \mathcal{G} t_{1}\left(f_{0}\langle\xi\rangle\right)$ since $\mathcal{G} t_{1}\left(f_{0}\langle\xi\rangle\right)$ is $C^{0}$-open in $\mathcal{G} t\left(f_{0}\langle\xi\rangle\right)$ (by Theorem 3.2.1).

2) $\mathcal{G} t_{1}(\omega)$ is $C^{0}$-dense in $\mathcal{G} t(\omega)$. Indeed, if $v \in \mathcal{G} t(\omega)$, then there exists an $\epsilon>0$ such that $v$ is an $\omega_{\epsilon}$-gradient. Choose any form $\omega^{\prime} \in \omega_{\epsilon}$ with $\left[\omega^{\prime}\right] \epsilon$ $H^{1}(M, \mathbf{Q})$. Then by Theorem 3.2.1 arbitrarily close to $v$ one can find an $\omega^{\prime}$-gradient $u \in \mathcal{G} t_{1}\left(f_{0}\left\langle\omega^{\prime}\right\rangle\right)$. By Lemma 4.2.4, $u$ can be chosen so as to be an $\omega_{\epsilon}$-gradient.

3) If $v \in \mathcal{G} t_{1}(\omega)$, then $\overline{\bar{n}}(\overline{\bar{x}}, \overline{\bar{y}} ; v) \in \mathfrak{s}_{[\omega]}^{-1} \mathbb{Z}\left[\mathbb{Z}^{m}\right]$. Indeed, $v$ is an $\omega$-gradient and a $\xi$-gradient for some $\xi \in \omega_{\epsilon}$ with $[\xi] \in H^{1}(M, \mathbf{Q})$. Note that if $[\xi]$ and $[\omega]$ are linearly dependent, then $[\xi]=\alpha[\omega]$ with $\alpha>0$. Indeed, $[\xi]=\alpha[\omega]$ with $\alpha<0$ would imply

$$
(1-\alpha)[\omega]+\sum \epsilon_{i} a_{i}^{*}=0
$$


which contradicts $\epsilon \leqslant\|[\omega]\| / 2$. Therefore Lemma 4.3 .5 implies that there is an integral $([\xi],[\omega])$-cone $\Delta$, such that

$$
\operatorname{supp} \overline{\bar{n}}(\overline{\bar{x}}, \overline{\bar{y}} ; v) \subset \Delta+b
$$

for some $b \in \mathbb{Z}^{m}$. Consider $\overline{\bar{n}}(\overline{\bar{x}}, \overline{\bar{y}} ; v)$ as an element of $\left(\mathbb{Z}\left[\mathbb{Z}^{m}\right]\right)_{[\xi]}^{-}$. Then Lemma 4.1.4 together with Theorem 3.2.1 imply that it belongs to the localization $\mathfrak{s}_{[\xi]}^{-1} \mathbb{Z}\left[\mathbb{Z}^{m}\right]$, therefore there are $P, Q \in \mathbb{Z}\left[\mathbb{Z}^{m}\right]$ such that

$$
P=Q \cdot \overline{\bar{n}}(\overline{\bar{x}}, \overline{\bar{y}} ; v) .
$$

Since $\overline{\bar{n}}(\overline{\bar{x}}, \overline{\bar{y}} ; v) \in \sigma_{\Delta}^{-1} \mathbb{Z}[\Delta]^{\wedge}$ the faithful flatness property imply

$$
\overline{\bar{n}}(\overline{\bar{x}}, \overline{\bar{y}} ; v) \in \sigma_{\Delta}^{-1} S_{\Delta}^{-1} \mathbb{Z}[\Delta] \subset \mathfrak{s}_{[\omega]}^{-1} \mathbb{Z}\left[\mathbb{Z}^{m}\right] .
$$

\section{An exAmple}

In this section we shall construct a Morse map $M \rightarrow S^{1}$ on a closed 3 -manifold $M$, having two critical points: $x$ of index 2 and $y$ of index 1 , such that

$$
n(\bar{x}, \bar{y} ; v)=\sum_{k \geqslant 0} n_{k} t^{k}
$$

with $n_{k} \sim \alpha \cdot \beta^{k}$ with $\alpha<0, \beta>0$. For any $f$-gradient $w$ sufficiently close to $v$ in $C^{0}$ topology, we shall have: $n_{k}(\bar{x}, \bar{y} ; w)=n_{k}(\bar{x}, \bar{y} ; v)$.

We start with a torus $T^{2}$, remove from it two open discs and obtain a surface $S$ with two components of boundary. Choose and fix a parallel $\beta$ and a meridian $\alpha$ of this twice punctured torus. The copies of this surface (resp. the copies of $\alpha, \beta$ etc.) will be denoted by the same letter $S$ (resp. $\alpha, \beta$, etc.) adding indices in order to distinguish between them. The corresponding discs will be denoted by $D_{1}, D_{2}$. We glue a copy of $S$, denoted by $S(1,1)$ to a copy of $S$, denoted by $S(1,2)$ and close the boundary by two discs $D_{1}(1), D_{1}(2)$. The resulting surface is called $N$. We glue three copies of $S$ successively, close the boundary and obtain a closed surface

$$
L=D_{1}\left(\frac{1}{2}\right) \cup S\left(\frac{1}{2}, 1\right) \cup S\left(\frac{1}{2}, 0\right) \cup S\left(\frac{1}{2}, 2\right) \cup D_{2}\left(\frac{1}{2}\right) .
$$

The surface $K=D_{1}(0) \cup S(0,1) \cup S(0,2) \cup D_{2}(0)$ is constructed similarly to $N$. The surface $L$ is depicted on Figure 5.1 below.

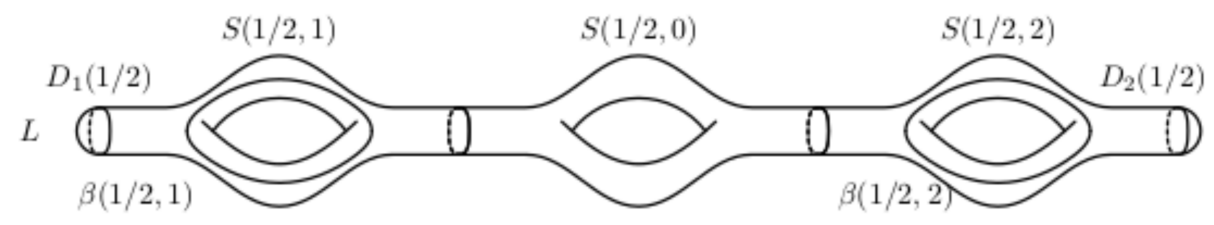

FIGURE 5.1. 
Let $W_{1}$ be the cobordism between $N$ and $L$, corresponding to the surgery on the circle $\beta\left(\frac{1}{2}, 2\right)$. Introduce on $W_{1}$ the corresponding Morse function $F_{1}: W_{1} \rightarrow\left[\frac{1}{2}, 1\right]$ with one critical point $x$ of index 2 , and such that

$$
F_{1}^{-1}(1)=N, \quad F_{1}^{-1}\left(\frac{1}{2}\right)=L .
$$

We can find an $F_{1}$-gradient $v_{1}$ such that

- $\left(-v_{1}\right)_{\left[\frac{1}{2}, 1\right]}^{\longrightarrow}$ restricted to

$$
D_{1}\left(\frac{1}{2}\right) \cup S\left(\frac{1}{2}, 1\right) \cup S\left(\frac{1}{2}, 0\right)
$$

is a diffeomorphism of this surface onto

$$
D_{1}(1) \cup S(1,1) \cup S(1,2)
$$

which identifies $S\left(\frac{1}{2}, 0\right)$ with $S(1,2)$ and $S\left(\frac{1}{2}, 1\right)$ with $S(1,1)$, slightly diminished from the left, so that the image of $D_{1}\left(\frac{1}{2}\right)$ contains $D_{1}(1)$ in its interior, and therefore

$$
\left(v_{1}\right)_{\left[\frac{1}{2}, 1\right]}^{\longrightarrow}\left(D_{1}(1)\right) \subset \operatorname{Int} D_{1}\left(\frac{1}{2}\right) ;
$$

- $\left(-v_{1}\right)_{\left[\frac{1}{2}, 1\right]}^{\longrightarrow}\left(S\left(\frac{1}{2}, 2\right) \cup D_{2}\left(\frac{1}{2}\right)\right)=D_{2}(1)$,

- $\left(-v_{1}\right)_{\left[\frac{1}{2}, 1\right]}^{\longrightarrow}\left(D_{2}\left(\frac{1}{2}\right)\right) \subset \operatorname{Int} D_{2}(1)$,

- and for some $\delta>0$ the $\left(-v_{1}\right)_{\left[\frac{1}{2}, 1\right]}$-image of a $\delta$-tubular neighborhood of $\beta\left(\frac{1}{2}, 2\right)$ is in $\operatorname{Int} D_{2}(1)$ (this image equals to $D_{\delta}\left(-v_{1}\right) \cap F_{1}^{-1}(1)$ ).

Let $W_{0}$ be the cobordism between $K$ and $L$, corresponding to the surgery on the circle $\beta\left(\frac{1}{2}, 1\right)$. Introduce on $W_{0}$ the corresponding Morse function $F_{0}: W_{0} \rightarrow\left[0, \frac{1}{2}\right]$ with one critical point $y$ of index 1 , and such that

$$
F_{0}^{-1}\left(\frac{1}{2}\right)=L, \quad F_{0}^{-1}(0)=K .
$$

We can find an $F_{0}$-gradient $v_{0}$ such that

- $\left(v_{0}\right)_{\left[\frac{1}{2}, 0\right]}^{\varkappa}$ restricted to

$$
S\left(\frac{1}{2}, 0\right) \cup S\left(\frac{1}{2}, 2\right) \cup D_{2}\left(\frac{1}{2}\right)
$$

is a diffeomorphpism of this surface onto

$$
D_{1}(0) \cup S(0,1) \cup S(0,2)
$$

which identifies $S\left(\frac{1}{2}, 0\right)$ with $S(0,1)$ and $S\left(\frac{1}{2}, 2\right)$ with $S(0,2)$ slightly diminished from the right so that the image of $D_{2}\left(\frac{1}{2}\right)$ contains $D_{2}(0)$ in its interior, and therefore

$$
\left(-v_{0}\right)_{\left[\frac{1}{2}, 0\right]}^{\hookrightarrow}\left(D_{2}(0)\right) \subset \operatorname{Int} D_{2}\left(\frac{1}{2}\right)
$$


- $\left(v_{0}\right)_{\left[\frac{1}{2}, 0\right]}^{m}\left(S\left(\frac{1}{2}, 1\right) \cup D_{1}\left(\frac{1}{2}\right)\right)=D_{1}(0)$;

- $\left(v_{0}\right)_{\left[\frac{1}{2}, 0\right]}^{m}\left(D_{1}\left(\frac{1}{2}\right)\right) \subset \operatorname{Int} D_{1}(0)$;

- and for some $\delta>0$ the $\left(v_{0}\right)_{\left[\frac{1}{2}, 0\right]}^{m \rightarrow}$-image of a $\delta$-tubular neighborhood of $\beta\left(\frac{1}{2}, 1\right)$ (this image equals to $\left.D_{\delta}\left(v_{0}\right) \cap F_{0}^{-1}(0)\right)$ is in $\operatorname{Int} D_{1}(0)$.

Glue together $W_{0}$ and $W_{1}$ along $L$, denote the resulting cobordism by $W$. Then we have $\partial W=K \cup N$. Glue the functions $F_{1}$ and $F_{0}$ to a Morse function $F: W \rightarrow[0,1]$ and the vector fields $v_{1}, v_{0}$ to an $F$-gradient $v$. We shall now define a ranging system for $(F, v)$. Consider the set $\Lambda=\left\{0, \frac{1}{2}, 1\right\}$ of regular values, and set

$$
\begin{array}{lll}
A_{1}=D_{1}(1), & A_{\frac{1}{2}}=D_{1}\left(\frac{1}{2}\right), & A_{0}=D_{1}(0), \\
B_{1}=D_{2}(1), & B_{\frac{1}{2}}=D_{2}\left(\frac{1}{2}\right), & B_{0}=D_{2}(0) .
\end{array}
$$

The fact that $\left\{\left(A_{\lambda}, B_{\lambda}\right)\right\}_{\lambda \in \Lambda}$ is a ranging system for $(F, v)$ follows immediately from the properties of $v_{0}, v_{1}$ cited above.

It is not difficult to compute the homomorphism

$$
H(v): H_{1}\left(N \backslash D_{2}(1), D_{1}(1)\right) \rightarrow H_{1}\left(K \backslash D_{2}(0), D_{1}(0)\right) .
$$

Namely,

$$
[\alpha(1,1)] \mapsto 0, \quad[\beta(1,1)] \mapsto 0, \quad[\alpha(1,2)] \mapsto[\alpha(0,1)], \quad[\beta(1,2)] \mapsto[\beta(0,1)]
$$

It is also obvious that the homology class of $D(x, v) \cap K$ equals to $[\beta(0,2)]$ and the homology class of $D(y,-v) \cap N$ equals to $[\beta(1,1)]$.

Consider the embedded curves

$$
\alpha(0,1), \quad \beta(0,1), \quad \alpha(0,2), \quad \beta(0,2)
$$

in $K$. Their homology classes form a symplectic basis in $K$. Consider the isomorphism of $H_{1}(K, \mathbb{Z}) \approx \mathbb{Z}^{4}$ given in this basis by the matrix

$$
\left(\begin{array}{cccc}
0 & 2 & 1 & 0 \\
0 & 0 & 0 & 1 \\
0 & 1 & 0 & 0 \\
-1 & 3 & 0 & -2
\end{array}\right)
$$

It preserves the intersection form, therefore it can be realized by a diffeomorphism $\Phi: K \rightarrow K$ and it is easy to see that we can assume that $\Phi(x)=x$ for $x \in D_{1}(0) \cup D_{2}(0)$. Denote by $\Psi$ the composition of $\Phi$ with the subsequent identification of $K$ with $N$.

Denote by $M$ the 3-dimensional manifold obtained by gluing of $K$ to $N$ by means of $\Psi$, and let $f: M \rightarrow S^{1}$ be the Morse function obtained from $F$. 
The corresponding cyclic covering $\bar{M}$ is a union of countably many copies of $W$, denoted by $W[i], i \in \mathbb{Z}$, glued together by the diffeomorphisms

$$
\Psi: K[i] \rightarrow N[i-1]
$$

of the components of boundaries.

The identification $W[k] \rightarrow W$ will be denoted by $J_{k}$. For $X \subset W$ we denote $J_{k}^{-1}(X)$ by $X[k]$. Define a lift $\bar{F}: \bar{M} \rightarrow \mathbb{R}$ of $f$ by setting

$$
\left.\bar{F}\right|_{W[k]}=F \circ J_{k}+k .
$$

Set

- $\Sigma=\{n / 2 \mid n \in \mathbb{Z}\}$

- $A_{n}=D_{1}(0)[n], B_{n}=D_{2}(0)[n]$ for $n \in \mathbb{Z}$;

- $A_{n}=D_{1}\left(\frac{1}{2}\right)[k], B_{n}=D_{2}\left(\frac{1}{2}\right)[k]$ for $n=k+\frac{1}{2}, k \in \mathbb{Z}$.

It is obvious that $\left\{\left(A_{\sigma}, B_{\sigma}\right)\right\}_{\sigma \in \Sigma}$ is a $t$-equivariant ranging system for $(\bar{F}, v)$. Put $\bar{x}=J_{0}^{-1}(x)$ and $\bar{y}=J_{0}^{-1}(y)$. Then we have:

$$
n_{0}(\bar{x}, \bar{y} ; v)=0, \quad n_{1}(\bar{x}, \bar{y} ; v)=\Psi_{*}([\beta(0,2)]) \sharp[\beta(1,1)]=0 .
$$

Denote by $D$ the matrix of $\Psi_{*} \circ H(v)$. Then

$$
D=\left(\begin{array}{cccc}
0 & 0 & 0 & 2 \\
0 & 0 & 0 & 0 \\
0 & 0 & 0 & 1 \\
0 & 0 & -1 & 3
\end{array}\right)
$$

Then for $k \geqslant 1$ we have

$$
n_{k+1}(\bar{x}, \bar{y} ; v)=\left(\Psi_{*} \circ H(v)\right)^{k}\left(\Psi_{*}([\beta(0,2)]) \sharp[\beta(1,1)] .\right.
$$

To abbreviate the notation we denote $[\alpha(1,1)]$ by $a_{1},[\alpha(1,2)]$ by $a_{2},[\beta(1,1)]$ by $b_{1},[\beta(1,2)]$ by $b_{2}$. Then $n_{k+1}(\bar{x}, \bar{y} ; v)$ is the coefficient of $a_{1}$ in

$$
D^{k}\left(b_{1}-2 b_{2}\right)=(-2) D^{k}\left(b_{2}\right) \text {. }
$$

To find $D^{k}\left(b_{2}\right)$ assume by induction that

$$
D^{k}\left(b_{2}\right)=\alpha_{k} a_{1}+\gamma_{k} a_{2}+\beta_{k} b_{2} .
$$

Then

$$
D^{k+1}\left(b_{2}\right)=-\gamma_{k} b_{2}+\beta_{l}\left(2 a_{1}+3 \beta_{2}+a_{2}\right)=2 \beta_{k} a_{1}+\left(3 \beta_{k}-\gamma_{k}\right) b_{2}+\beta_{k} a_{2} .
$$

Therefore the vectors $\left(\beta_{k}, \gamma_{k}\right)$ satisfy

$$
\left(\begin{array}{c}
\beta_{k+1} \\
\gamma_{k+1}
\end{array}\right)=\left(\begin{array}{cc}
3 & -1 \\
1 & 0
\end{array}\right)\left(\begin{array}{l}
\beta_{k} \\
\gamma_{k}
\end{array}\right) \text { and } \quad\left(\begin{array}{l}
\beta_{0} \\
\gamma_{0}
\end{array}\right)=\left(\begin{array}{l}
1 \\
0
\end{array}\right)
$$


The coefficient $\alpha_{k+1}=2 \beta_{k}$. Denote

$$
\left(\begin{array}{cc}
3 & -1 \\
1 & 0
\end{array}\right)
$$

by $C$. Then the explicit computation shows

$$
C^{n}=\frac{1}{\sqrt{5}}\left(\begin{array}{cc}
\lambda_{1}^{n+1}-\lambda_{2}^{n+1} & \lambda_{2}^{n}-\lambda_{1}^{n} \\
\lambda_{1}^{n}-\lambda_{2}^{n} & \lambda_{2}^{n-1}-\lambda_{1}^{n-1}
\end{array}\right),
$$

where $\lambda_{1}=\frac{3+\sqrt{5}}{2}, \quad \lambda_{2}=\frac{3-\sqrt{5}}{2}$. Therefore

$$
n_{k+1}(\bar{x}, \bar{y} ; v)=-\frac{4}{\sqrt{5}}\left(\left(\frac{3+\sqrt{5}}{2}\right)^{k}-\left(\frac{3-\sqrt{5}}{2}\right)^{k}\right) .
$$

\subsection{Incidence coefficients with respect to the universal cover: the exponential estimate.}

Lemma 5.1.1. Let $\xi, \eta: \mathbb{R}^{m} \rightarrow \mathbb{R}$ be non-zero linear forms, and let $\Gamma$ be a $(\xi, \eta)$-integral cone. Then there is $A>0$ such that for every $b \in \mathbb{R}^{m}$ there is $B \in \mathbb{R}$ such that for every $c \in \mathbb{R}$ we have:

$$
(\Gamma+b) \cap \xi^{-1}([c, \infty)) \subset(\Gamma+b) \cap \eta^{-1}([A c+B, \infty)) .
$$

Proof. Abbreviate $\xi^{-1}([c, \infty))$ by $\{\xi \geqslant c\}$. It is sufficient to consider the case $b=0$, that is, to prove that there is $A \geqslant 0$ such that

$$
\Gamma \cap\{\xi \geqslant c\} \subset \Gamma \cap\{\eta \geqslant A c\}
$$

(the property (5.1) with arbitrary $b$ follows then from $B=\eta(b)-A \xi(b)$ ).

To prove (5.2) let $e_{i}$ be the generators of $\Gamma$, and choose $A>0$ such that

$$
\eta\left(e_{i}\right) \geqslant A \xi\left(e_{i}\right)
$$

for all $i$. Then $x \in \Gamma \cap\{\xi \geqslant c\}$ means that $x=\sum \lambda_{i} e_{i}$, where $\lambda_{i} \geqslant 0$ and $\sum \lambda_{i} \xi\left(e_{i}\right) \geqslant c$. This implies

$$
\eta(x)=\sum \lambda_{i} \eta\left(e_{i}\right) \geqslant \sum \lambda_{i} A \xi\left(e_{i}\right) \geqslant A \xi(x) .
$$

Definition 5.1.2. Let $\lambda=\sum_{g} n_{g} g \in \mathbb{Z}\left[\pi_{1} M\right]_{\xi}^{-}$and $c \in \mathbb{R}$. Put

$$
\lambda[c]=\sum_{\xi(g) \geqslant c} n_{g} \cdot g \in \mathbb{Z}\left[\pi_{1} M\right], \quad N_{c}(\lambda)=\sum_{\xi(g) \geqslant c}\left|n_{g}\right| .
$$


5.2. Proof of Theorem C. By the definition of $\mathcal{G} t_{1}(\omega)$ there exists an

$$
\epsilon \in\left(0, \frac{1}{2}\|[\omega]\|\right)
$$

and a Morse form $\xi \in \omega_{\epsilon}$, such that

$$
[\xi] \in H^{1}(M, \mathbf{Q}), \quad v \in \mathcal{G} t_{1}\left(f_{0}\langle\xi\rangle\right) .
$$

Therefore there is an integral $([\xi],[\omega])$-cone $\Delta$ and $b \in \mathbb{Z}^{m}$, such that

$$
Q(\operatorname{supp} n(\widetilde{x}, \widetilde{y} ; v)) \subset \Delta+b .
$$

Lemma 5.1.1 implies then that

$$
\begin{aligned}
\operatorname{supp} \tilde{n}(\tilde{x}, \tilde{y} ; v) & \cap(\{\omega\})^{-1}([c, \infty)) \\
& \subset \operatorname{supp} \tilde{n}(\widetilde{x}, \tilde{y} ; v) \cap(\{\xi\})^{-1}([A c+B, \infty)),
\end{aligned}
$$

and since $v$ is a $\xi$-gradient, our estimate follows from Theorem 3.2.1 and Proposition 3.1.3.

5.3. On the Novikov complex for a Morse form. In this paper we do not use the notion of the Novikov complex, and work only with the incidence coefficients. The latter were introduced however in [9] as the matrix entries of the boundary operators in the Novikov complex. In this subsection we use the results of Subsection 4.2 and 5.2 to give a simple proof of the fact that the boundary operators above indeed turn the graded module into a chain complex (that is, $\partial^{2}=0$ ). We reduce the proof to the corresponding statement about rational Morse forms, proved in [12].

We assume here the terminology of Subsection 1.2 of the Introduction. Moreover, if $\Delta$ is an integral $[\omega]$-cone, we denote by $\lambda_{\Delta}$ the subset of $\mathbb{Z}\left[\pi_{1} M\right]_{\{\omega\}}^{-}$, defined by

$$
\lambda_{\Delta}=\left\{\lambda \mid \text { there exists } b \in \mathbb{Z}^{m} \text { such that } Q(\operatorname{supp} \lambda) \subset \Delta+b\right\} .
$$

It is not difficult to see that $\lambda_{\Delta}$ is a subring of $\mathbb{Z}\left[\pi_{1} M\right]_{\{\omega\}}^{-}$.

Now let $v$ be an $\omega$-gradient satisfying transversality assumption and $\epsilon>0$ so small that $\omega_{\epsilon}$ is a Morse family and $v$ is an $\omega_{\epsilon}$-gradient. Let $\xi \in \omega_{\epsilon}$ be a Morse form such that $[\xi] \in H^{1}(M, \mathbf{Q})$. Then it follows from Lemma 4.3.5 that there is an integral $([\xi],[\omega])$-cone $\Delta$, such that for every $x, y \in S(\omega)$ with ind $x=$ ind $y+1$ we have

$$
\widetilde{n}(\widetilde{x}, \widetilde{y} ; v) \subset \lambda_{\Delta}
$$

Therefore the homomorphism

$$
\partial: C_{*}(\omega, v) \rightarrow C_{*-1}(\omega, v)
$$

is defined actually over the ring $\lambda_{\Delta}$ and to verify that $\partial^{2}=0$ it is sufficient to verify it over the ring $\mathbb{Z}\left[\pi_{1} M\right]_{\{\xi\}}^{-}$, and this is done in [12]. 


\section{Exponential estimates of the ABSOlute NUMBER OF TRAJECTORIES}

6.1. Bunches of ranging systems. Let $f: W \rightarrow[a, b]$ be a Morse function on a cobordism $W$ such that

$$
f^{-1}(b)=V_{1}, \quad f^{-1}(a)=V_{0}, \quad \operatorname{dim} W=n .
$$

Let $v$ be an $f$-gradient, $\Lambda=\left\{\lambda_{0}, \ldots, \lambda_{k}\right\}$ be a set of regular values of $f$, such that

$$
\lambda_{0}=a<\lambda_{1}<\ldots<\lambda_{k-1}<\lambda_{k}=b
$$

and between any two adjacent values $\lambda_{i}$ and $\lambda_{i+1}$ there is exactly one critical value of $f$.

Definition 6.1.1. Assume that for each integer $s: 0 \leqslant s \leqslant n$ and every $\lambda \in \Lambda$ there are given compacts $A_{\lambda}^{(s)}, B_{\lambda}^{(s)}$ in $f^{-1}(\lambda)$. We shall say that

$$
\left\{\left(A_{\lambda}^{(s)}, B_{\lambda}^{(s)}\right)\right\}_{\lambda \in \Lambda, 0 \leqslant s \leqslant n}
$$

is a bunch of ranging systems for $(f, v)$ (abbreviation: BRS for $(f, v))$ if the following conditions hold:

(1) $A_{\lambda}^{(0)}=B_{\lambda}^{(0)}=\varnothing$.

(2) $r \leqslant s \Rightarrow\left(A_{\lambda}^{(r)} \subset A_{\lambda}^{(s)}, B_{\lambda}^{(r)} \subset B_{\lambda}^{(s)}\right)$.

(3) $A_{\lambda}^{(r)} \cap B_{\lambda}^{(s)}=\varnothing$ for $r+s \leqslant n$,

(4) If $\lambda, \mu \in \Lambda$ are adjacent, then for every $r, s$ :

$$
v_{[\mu, \lambda]}^{m \rightarrow}\left(A_{\mu}^{(r)}\right) \subset \operatorname{Int} A_{\lambda}^{(r)} \quad \text { and } \quad(-v)_{[\lambda, \mu]}^{m \rightarrow}\left(B_{\lambda}^{(s)}\right) \subset \operatorname{Int} B_{\mu}^{(s)} .
$$

(5) Let $\lambda, \mu \in \Lambda$ be adjacent. Then for every $p \in S(f) \cap f^{-1}([\lambda, \mu])$ we have:
i) $D(p, v) \cap f^{-1}(\lambda) \subset \operatorname{Int} A_{\lambda}^{(\operatorname{ind} p)}$,
ii) $D(p,-v) \cap f^{-1}(\mu) \subset \operatorname{Int} B_{\mu}^{(n-\operatorname{ind} p)}$.

Let $\left\{\left(A_{\lambda}^{(s)}, B_{\lambda}^{(s)}\right)\right\}_{\lambda \in \Lambda, 0 \leqslant s \leqslant n}$ be a BRS for $(f, v)$. The following properties are either obvious or proved similarly to the corresponding properties of ranging systems (see Subsection 2.2 and $[15, \S 4]$ ).

i) For every $s: 0 \leqslant s \leqslant n$ the family $\left\{\left(A_{\lambda}^{(s)}, B_{\lambda}^{(n-s-1)}\right)\right\}_{\lambda \in \Lambda}$ is a ranging system for $(f, v)$.

ii) $v$ is an almost good $f$-gradient.

iii) There is $\epsilon>0$ such that for every $f$-gradient $w$ with $\|w-v\|<\epsilon$ the system $\left\{\left(A_{\lambda}^{(s)}, B_{\lambda}^{(s)}\right)\right\}_{\lambda \in \Lambda, 0 \leqslant s \leqslant n}$ is a BRS for $(f, w)$. 
iv) For every $\delta>0$ sufficiently small the following strengthening of (5) holds:

$$
\left\{\begin{array}{l}
D_{\delta}(p, v) \cap f^{-1}(\lambda) \subset \operatorname{Int} A_{\lambda}^{(\operatorname{ind} p)}, \\
D_{\delta}(p,-v) \cap f^{-1}(\mu) \subset \operatorname{Int} B_{\mu}^{(n-\operatorname{ind} p)} .
\end{array}\right.
$$

For $p \in S(f)$ we shall denote by $\lambda_{p}$ and $\mu_{p}$ the adjacent elements of $\Lambda$ such that $\lambda_{p}<f(p)<\mu_{p}$. Choose $\rho>0$ so small that for every $p \in S(f)$ we have:

$$
\lambda_{p}<\lambda_{p}+\rho<f(p)-\rho<f(p)+\rho<\mu_{p}-\rho .
$$

Let $\delta>0$ satisfy (6.1) above and assume further that

$$
\left\{\begin{array}{l}
(1) \text { If } p, q \in S(f) \text { and } f(p)=f(q) \text { then: } \\
D_{\delta}(p, v) \cap D_{\delta}(q, v) \cap f^{-1}\left(\left[\lambda_{p}, \mu_{p}\right]\right)=\varnothing \\
D_{\delta}(p,-v) \cap D_{\delta}(q,-v) \cap f^{-1}\left(\left[\lambda_{p}, \mu_{p}\right]\right)=\varnothing .
\end{array}\right.
$$

(2) For every $p \in S(f)$ we have

$$
D_{\delta}(p) \subset f^{-1}(] f(p)-\rho, f(p)+\rho[) .
$$

Let $\epsilon>0$ satisfy iii) above. The manifold

$$
S_{p}=D(p,-v) \cap f^{-1}\left(\mu_{p}\right)
$$

is an embedded sphere of dimension $n-$ ind $p-1$. The normal bundle to $S_{p}$ in $f^{-1}\left(\mu_{p}\right)$ is trivial. Chose an embedding

$$
\psi: S_{p} \times B^{\text {ind } p}(0, \theta) \rightarrow f^{-1}\left(\mu_{p}\right)
$$

(where $\theta>0$ ) which is a diffeomorphism onto its image, and such that $\left.\psi\right|_{\left(S_{p} \times\{0\}\right)}=\mathrm{id}$.

For $\varkappa \in(0 ; \theta]$ the set $\psi\left(S_{p} \times B^{\text {ind }} p(0, \varkappa)\right)$ will be denoted by $\operatorname{Tub}\left(S_{p}, \varkappa\right)$ and for $\eta \in B^{\text {ind }} p(0, \theta)$ the embedded sphere $\psi\left(S_{p} \times\{\eta\}\right)$ by $S_{p}(\eta)$. Assume that $\theta$ is so small that

$$
\overline{\operatorname{Im} \psi} \subset B_{\delta}(p,-v) .
$$

Choose $\tau>0$ so small that for every $z \in f^{-1}\left(\mu_{p}\right)$ the $v$-trajectory $\gamma(z, \cdot ; v)$ is defined on the interval $[-\tau, 0]$ and its image is in $f^{-1}\left(\left(\mu_{p}-\rho, \mu_{p}\right]\right)$. Define a map

$$
\Psi:[-\tau, 0] \times S_{p} \times B^{\text {ind } p}(0, \theta) \rightarrow W
$$

by

$$
\Psi(t, s, h)=\gamma(\psi(s, h), t ; v) .
$$

Then $\Psi$ is a diffeomorphism onto its image, and the vector field $\Psi_{*}^{-1}(v)$ equals $(1,0,0)$.

Let $\xi \in B^{\text {ind } p}\left(0, \frac{\theta}{2}\right)$ and let $H_{t}\langle\xi\rangle, t \in[-\tau, 0]$, be an isotopy of $B^{\text {ind } p}(0, \theta)$ having the following properties:

(1) $\frac{d}{d t} H_{t}\langle\xi\rangle(x)=0$ for $t \in\left[-\tau,-\frac{2}{3} \tau\right] \cup\left[-\frac{1}{3} \tau, 0\right]$. 
(2) $H_{t}\langle\xi\rangle(x)=x$ for $x \in B^{\text {ind } p}(0, \theta) \backslash B^{\text {ind } p}\left(0, \frac{\theta}{2}\right)$, and for $t \in\left[-\tau,-\frac{2}{3} \tau\right]$.

(3) $H_{0}\langle\xi\rangle(0)=\xi$.

Then $\frac{d}{d t} H_{t}\langle\xi\rangle$ is a time-dependent vector field on $B^{\text {ind } p}(0, \theta)$. Define a vector field $w\langle\xi\rangle$ on $[-\tau, 0] \times S_{p} \times B^{\text {ind } p}(0, \theta)$ by

$$
w\langle\xi\rangle(t, x, y)=\left(1,0, \frac{d}{d t} H_{t}\langle\xi\rangle\right) .
$$

Remark 6.1.2. Observe that for every $x \in S_{p} \times B^{\text {ind } p}\left(0, \frac{\theta}{2}\right)$ we have

$$
\gamma(x,-\tau ; w\langle\xi\rangle) \in\{-\tau\} \times S_{p} \times B^{\text {ind } p}\left(0, \frac{\theta}{2}\right) .
$$

Choose $\alpha \in\left(0, \frac{\theta}{2}\right)$ so small that for each $\xi \in B^{\text {ind } p}(0, \alpha)$ there is an isotopy $H_{t}\langle\xi\rangle$ such that

$$
\left\|v-\Psi_{*}(w\langle\xi\rangle)\right\|<\epsilon .
$$

We perform this construction for every $p \in S(f)$, and obtain a diffeomorphism $\Psi_{p}$, a vector $\xi_{p}$, and a vector field $w_{p}\left\langle\xi_{p}\right\rangle$. We assume that $\theta_{p}, \alpha_{p}, \tau_{p}$ are chosen to be independent of $p$, and we denote them by $\theta, \alpha, \tau$ omitting $p$ in the notation. The set $\left\{\xi_{p}\right\}_{p \in S(f)}$ will be denoted by $\vec{\xi}$. Define a new vector field $u=v\langle\vec{\xi}\rangle$ setting

$$
u(x)= \begin{cases}v(x) & \text { for } x \in W \backslash \underset{p}{\cup} \operatorname{Im} \Psi_{p}, \\ \left(\Psi_{p}\right)_{*}\left(w_{p}\left\langle\xi_{p}\right\rangle\right)(x) & \text { for } x \in \operatorname{Im} \Psi_{p} .\end{cases}
$$

It follows from the construction that for every $\vec{\xi}$ the vector field $v\langle\vec{\xi}\rangle$ is an $f$-gradient. Note also that for $p \in S(f)$ we have

$$
D(p,-v\langle\vec{\xi}\rangle) \cap f^{-1}\left(\mu_{p}\right)=S_{p}\left(\xi_{p}\right) .
$$

The condition (6.4) implies that $\left\{\left(A_{\lambda}^{(s)}, B_{\lambda}^{(s)}\right)\right\}_{\lambda \in \Lambda, 0 \leqslant s \leqslant n}$ is a BRS for $(f, v\langle\vec{\xi}\rangle)$. Therefore $v\langle\vec{\xi}\rangle$ is an almost good $f$-gradient. It will be called regular perturbation of $v$ corresponding to $\vec{\xi}$.

Proposition 6.1.3. 1) Let $p, q \in S(f)$ with ind $p=$ ind $q+1$. The set of $(-v\langle\vec{\xi}\rangle)$-trajectories ${ }^{6}$ joining $p$ with $q$ is in a bijective correspondence with the set

$$
\left(D(p, v) \cap f^{-1}\left(\mu_{q}\right)\right) \cap S_{q}\left(\xi_{q}\right) .
$$

2) The vector field $v\langle\vec{\xi}\rangle$ is a good $f$-gradient if for every $p, q \in S(f)$ with ind $p=$ ind $q+1$ the submanifold $D(p, v) \cap f^{-1}\left(\mu_{q}\right)$ of $f^{-1}\left(\mu_{q}\right)$ is transversal to $S_{q}\left(\xi_{q}\right)$.

\footnotetext{
${ }^{6}$ We identify here two trajectories which differ by a parameter change.
} 
Proof. 1) Let $\gamma$ be a $(-v\langle\vec{\xi}\rangle)$-trajectory joining $p$ with $q$ and $\gamma\left(t_{0}\right) \inf ^{-1}\left(\mu_{q}\right)$, where $t_{0} \in \mathbb{R}$. I claim that for $t<t_{0}$ we have

$$
\gamma(t) \notin \operatorname{supp}(v-v\langle\vec{\xi}\rangle)
$$

Indeed, if the opposite is true, let $t_{1}<t_{0}$ be the first moment when $\gamma$ intersect $\operatorname{supp}(v-v\langle\vec{\xi}\rangle)$. Then there is $s \in S(f) \operatorname{such}$ that $\gamma\left(t_{1}\right) \in \operatorname{Tub}\left(S_{s}, \frac{\theta}{2}\right)$. Note that

$$
\operatorname{Tub}\left(S_{s}, \frac{\theta}{2}\right) \subset D_{\delta}(s,-v) \cap f^{-1}\left(\mu_{s}\right) \subset \operatorname{Int} B_{\mu_{s}}^{(n-\operatorname{ind} s)},
$$

therefore $\gamma\left(t_{1}\right)$ belongs to the intersection of $A_{\mu_{s}}^{(\operatorname{ind} p)}$ with $B_{\mu_{s}}^{(n-\text { ind } s)}$ and ind $p \geqslant$ ind $s+1$.

Further, $f(s)>f(q)$ and $\lambda_{s} \geqslant \mu_{q}$. Denote by $t_{2}$ the moment when $\gamma$ intersects $f^{-1}\left(\lambda_{s}\right)$. Then $t_{0} \geqslant t_{2}>t_{1}+\tau$, and it is easy to see that $\left.\gamma\right|_{\left[t_{1}+\tau, t_{2}\right]}$ does not intersect $\operatorname{supp}(v-v\langle\vec{\xi}\rangle)$. The property (6.3) implies that

$$
\gamma\left(t_{1}+\tau\right) \in D_{\delta}(s,-v)
$$

and, therefore,

$$
\gamma\left(t_{2}\right) \in D_{\delta}(s, v) \cap f^{-1}\left(\lambda_{s}\right) \subset \operatorname{Int} A_{\lambda_{s}}^{(\text {ind } s)}
$$

which implies $\gamma\left(t_{0}\right) \in \operatorname{Int} A_{\mu_{q}}^{(\text {ind } s)}$. Hence

$$
A_{\mu_{q}}^{(\text {ind } s)} \cap D_{\delta}(q,-v) \neq \varnothing
$$

and thus

$$
A_{\mu_{q}}^{(\text {ind } s)} \cap B_{\mu_{q}}^{(n-\operatorname{ind} q)} \neq \varnothing
$$

which implies

$$
\text { ind } s>\operatorname{ind} q \text { and } \quad \text { ind } p \geqslant \text { ind } s+1>\text { ind } q+1 .
$$

This gives a contradiction.

Therefore $\left.\gamma\right|_{\left(-\infty, t_{0}\right]}$ is a $(-v)$-trajectory. In the same way one can show that every $(-v)$-trajectory joining $p$ with a point of $D_{\delta}(q,-v) \cap f^{-1}\left(\mu_{q}\right)$ does not intersect $\operatorname{supp}(v-v\langle\vec{\xi}\rangle)$. That proves the property 1$)$.

2 ) Note that $v\langle\vec{\xi}\rangle$ is almost good. Therefore, $v\langle\vec{\xi}\rangle$ is good if and only if

$$
(\text { ind } p=\operatorname{ind} q+1) \Rightarrow(D(p, v\langle\vec{\xi}\rangle) \pitchfork D(q,-v\langle\vec{\xi}\rangle)) \text {. }
$$

Let $p, q \in S(f)$, ind $p=$ ind $q+1$. It suffices to prove that the intersection $D(p, v\langle\vec{\xi}\rangle) \cap f^{-1}\left(\mu_{q}\right)$ is transversal to $S_{q}\left(\xi_{q}\right)$. Let $x$ be a point in the intersection of these manifolds. In the part 1) we have proved that there is a $(-v)$-trajectory, joining $p$ with $x$ and not intersecting $\operatorname{supp}(v-v\langle\vec{\xi}\rangle)$. Then 
a small neighborhood of this trajectory does not intersect $\operatorname{supp}(v-v\langle\vec{\xi}\rangle)$ and the transversality sought follows from

$$
\left(D(p, v) \cap f^{-1}\left(\mu_{q}\right)\right) \pitchfork S_{q}\left(\xi_{q}\right) .
$$

6.2. Volume estimates. We will use here the terminology of the previous subsection. Let

$$
\left\{\left(A_{\lambda}^{(s)}, B_{\lambda}^{(s)}\right)\right\}_{\lambda \in \Lambda, 0 \leqslant s \leqslant n}
$$

be a BRS for $(f, v)$. Assume that $\delta>0$ satisfies (6.2) from Subsection 6.1. Fix an integer $s: 0 \leqslant s \leqslant n$. Let $\lambda<\mu$ be adjacent elements of $\Lambda$. Denote by $S^{\prime}$ the subset of all critical points of $f$ of index $\leqslant s$ and with critical values in $(\lambda, \mu)$. The set

$$
\mathcal{D}_{\mu}^{(s)}=f^{-1}(\mu) \backslash\left(\operatorname{Int} A_{\mu}^{(s)} \cup \operatorname{Int} B_{\mu}^{(n-s-1)} \cup\left(\cup_{p \in S^{\prime}} B_{\delta}(p,-v) \cap f^{-1}(\mu)\right)\right)
$$

is a compact subset of the domain of definition of $v_{[\mu, \lambda]}^{\varkappa}$. Denote by $N_{\mu}^{(s)}$ the norm of the derivative of $v_{[\mu, \lambda]}^{\leadsto}$ restricted to $\mathcal{D}_{\mu}^{(s)}$ :

$$
N_{\mu}^{(s)}=\sup _{x \in \mathcal{D}_{\mu}^{(s)}}\left\|\left(v_{[\mu, \lambda]}^{\sim m}\right)^{\prime}(x)\right\|
$$

and by $A$ the maximum of $\left(N_{\mu}^{(s)}\right)^{k}$ over all $\mu \in \Lambda, 0 \leqslant s \leqslant n$, and $0 \leqslant k \leqslant n$. For $p \in S(f)$ denote by $B_{(p)}$ the norm of the derivative of

$$
\left.\psi_{p}^{-1}\right|_{\psi_{p}\left(S_{p} \times D^{\text {ind }} p\left(0, \frac{\theta}{2}\right)\right)}
$$

(where the manifold $S_{p} \times B^{\text {ind }} p(0, \theta)$ is endowed with the product Riemannian metric). Denote by $B$ the maximum of $B_{(p)}$ over all $p \in S(f)$.

Lemma 6.2.1. Let $\lambda, \mu \in \Lambda, \lambda<\mu$, and $s$ be an integer with $0 \leqslant s \leqslant n$. Let also $N$ be a submanifold of $f^{-1}(\mu) \backslash B_{\mu}^{(n-s-1)}$, such that $N \backslash A_{\mu}^{(s)}$ is compact. Denote by $k$ the number of elements of $\Lambda$ belonging to $[\lambda, \mu]$. Then $N^{\prime}=v_{[\mu, \lambda]}^{\hookrightarrow}(N)$ is a submanifold of $f^{-1}(\lambda) \backslash B_{\lambda}^{(n-s-1)}$, such that $N^{\prime} \backslash A_{\lambda}^{(s)}$ is compact, and

1) $\operatorname{vol}\left(N^{\prime} \backslash A_{\lambda}^{(s)}\right) \leqslant A^{k-1} \cdot \operatorname{vol}\left(N \backslash A_{\mu}^{(s)}\right)$;

2) if $p \in S(f)$ with ind $p=s$ and $\mu_{p}=\lambda$, then

$$
\operatorname{vol}\left(\psi_{p}^{-1}\left(N^{\prime} \cap \operatorname{Tub}\left(S_{p}, \frac{\theta}{2}\right)\right) \leqslant B A^{k-1} \operatorname{vol}\left(N \backslash A_{\mu}^{(s)}\right) .\right.
$$

Proof. For the proof of 1) note that it suffices to consider the case $k=2$. For this case we have obviously $N^{\prime} \backslash A_{\lambda}^{(s)} \subset \stackrel{m}{v}\left(N \cap \mathcal{D}_{\mu}^{(s)}\right)$. Statement 2) follows from 1) since $\operatorname{Tub}\left(S_{p}, \frac{\theta}{2}\right) \subset B_{\delta}(p,-v)$ does not intersect $A_{\lambda}^{(s)}$. 
6.3. Bunches of equivariant ranging systems. We will use here the terminology of $\S 1.3 .1$ of Introduction. Assume that the homotopy class of $f$ is indivisible, so that $F(x t)=F(x)-1$.

Definition 6.3.1. Let $\Sigma$ be the set of regular values of $F$ such that

( $\Sigma 1) \sigma \in \Sigma \Rightarrow \sigma+n \in \Sigma$ for every $n \in \mathbb{Z}$.

$(\Sigma 2)$ If $\sigma_{1}, \sigma_{2} \in \Sigma$ are adjacent, there is exactly one critical value of $F$ in $\left(\sigma_{1}, \sigma_{2}\right)$.

( $\Sigma 3)$ For every $A, B \in \mathbb{R}$ the set $\Sigma \cap[A, B]$ is finite.

Assume that for every integer $s: 0 \leqslant s \leqslant n$ and every $\sigma \in \Sigma$ there are given compacts $A_{\sigma}^{(s)}, B_{\sigma}^{(s)}$ in $F^{-1}(\sigma)$. We shall say that

$$
\left\{\left(A_{\sigma}^{(s)}, B_{\sigma}^{(s)}\right)\right\}_{\sigma \in \Sigma, 0 \leqslant s \leqslant n}
$$

is a bunch of t-equivariant ranging systems (abbreviation: BERS for $(F, v)$ ) if:

(1) For every $\mu, \nu \in \Sigma, \mu<\nu$ the system $\left\{\left(A_{\sigma}^{(s)}, B_{\sigma}^{(s)}\right)\right\}_{\sigma \in \Sigma, \mu \leqslant \sigma \leqslant \nu}$ is a $\operatorname{BRS}$ for $\left(\left.F\right|_{\left.F^{-1}([\mu, \nu]), v\right)}\right.$;

(2) $A_{\sigma-n}^{(s)}=A_{\sigma}^{(s)} \cdot t^{n}$ and $B_{\sigma-n}^{(s)}=B_{\sigma}^{(s)} \cdot t^{n}$ for every $n \in \mathbb{Z}$.

$\mathrm{Up}$ to the end of this subsection we assume that $(F, v)$ has a BERS $\left\{\left(A_{\sigma}^{(s)}, B_{\sigma}^{(s)}\right)\right\}_{\sigma \in \Sigma, 0 \leqslant s \leqslant n}$.

Choose any $\sigma \in \Sigma$ and denote by $W$ the cobordism $F^{-1}([\sigma, \sigma+1])$ and by $\Lambda$ the set $\Sigma \cap[\sigma, \sigma+1]$. We apply the constructions of Subsections 6.1 and 6.2 to $W$ and then extend the results to the whole of $\bar{M}$ in the $t$-invariant way, thus obtaining the sets $\operatorname{Tub}\left(S_{q}, \varkappa\right) \subset F^{-1}\left(\mu_{q}\right)$ for every $q \in S(F)$. Let $p, q \in S(F)$. Assume that $F(p)>F(q)$ and ind $p=$ ind $q+1$. Denote by $N_{p, q}$ the submanifold of dimension ind $q$ of $S_{q} \times B^{\text {ind } q}\left(0, \frac{\theta}{2}\right)$, defined by

$$
N_{p, q}=\psi_{q}^{-1}\left(\left(D(p, v) \cap F^{-1}\left(\mu_{q}\right)\right) \cap\left(\operatorname{Tub}\left(S_{q}, \frac{\theta}{2}\right)\right)\right) .
$$

The next lemma follows from Lemma 6.2.1.

Lemma 6.3.2. There are constants $C, D>0$ such that for every $p, q \in$ $S(F)$ with ind $p=$ ind $q+1$ and $F(p)>F(q)$ we have:

$$
\operatorname{vol}\left(N_{p, q}\right) \leqslant C \cdot D^{[F(p)-F(q)]} .
$$

The next lemma is a direct consequence of Sard Theorem.

Lemma 6.3.3. Let $q \in S(F)$. Then there is a residual subset

$$
Q \subset B^{\text {ind } q}(0, \alpha)
$$


such that for every $\xi \in Q$ and every $p \in S(F)$ with ind $p=$ ind $q+1$ we have: $D(p, v) \pitchfork S_{q}(\xi)$.

The next proposition follows from (5.2) by arguments due to V. I. Arnold (see [1, p. 81]).

Proposition 6.3.4. Let $q \in S(F)$. Then there is a subset $Q \subset B^{\operatorname{ind} q}(0, \alpha)$ of full measure such that for every $\xi \in Q$ we have:

- For every $p \in S(F)$ with ind $p=$ ind $q+1$ there are constants $K, R>0$, such that for every integer $l \geqslant 0$ we have:

$$
\sharp\left(N_{p t^{-l}, q} \cap\left(S_{q} \times\{\xi\}\right)\right) \leqslant K \cdot R^{l} .
$$

Corollary 6.3.5. Let $\nu>0$. Then there exists a good $f$-gradient $u$ with $\|u-v\|<\nu$ and constants $K, L>0$ such that for every $p, q \in S(f)$ with ind $p=$ ind $q+1$ we have $N_{l}(p, q ; v) \leqslant L \cdot K^{l}$.

Proof. The construction of regular perturbation, described in $\S 6.1$, applied to $\left.v\right|_{W}$, gives for every $\vec{\xi}=\left\{\xi_{p}\right\}_{p \in S(F) \cap W}, \xi_{p} \in B^{\text {ind } p}(0, \alpha)$, an $\left.F\right|_{W}$-gradient $v\langle\vec{\xi}\rangle$. Since $\operatorname{supp}(v-v\langle\vec{\xi}\rangle)$ does not intersect $\partial W$, we can extend $v\langle\vec{\xi}\rangle t$ invariantly to $\bar{M}$ and obtain a $t$-invariant $F$-gradient, which will be denoted by the same symbol $v\langle\vec{\xi}\rangle$. Note that since

$$
\left\{\left(A_{\sigma}^{(s)}, B_{\sigma}^{(s)}\right)\right\}_{\sigma \in \Sigma, 0 \leqslant s \leqslant n}
$$

is a BERS for $(F, v\langle\vec{\xi}\rangle)$, the vector field $v\langle\vec{\xi}\rangle$ is an almost good $F$-gradient.

If all $\xi_{q} \in B^{\text {ind } q}(0, \alpha)$ are sufficiently small, we will have $\|u-v\langle\vec{\xi}\rangle\|<\nu$. Lemma 6.3.3 and Proposition 6.3.4 imply that we can choose the components $\xi_{q}$ of $\vec{\xi}$ in such a way that

(1) For every $p \in S(F)$,ind $p=$ ind $q+1$ we have:

$$
D(p, v) \cap F^{-1}\left(\mu_{q}\right) \pitchfork S_{q}\left(\xi_{q}\right) .
$$

(2) There are $K, R>0$ such that for every $p \in S(F)$ with ind $p=$ ind $q+1$ we have:

$$
\sharp\left(D\left(p t^{-l}, v\right) \cap S_{q}\left(\xi_{q}\right)\right) \leqslant K \cdot R^{l} .
$$

Apply Proposition 6.1.3 and deduce that $u=v\langle\vec{\xi}\rangle$ satisfy the conclusions of the Corollary.

6.4. Proof of Theorem D. We can assume that $S(f) \neq 0$ and that the homotopy class of $f$ is indivisible. In view of Corollary 6.3.5 it is sufficient to prove that the set of $f$-gradients, having a BERS, is $C^{0}$-dense in $\mathcal{G}(f)$. To prove that, let $v$ be any $f$-gradient and $\epsilon>0$. Choose any regular value $\lambda$ of $F$ and denote by $W$ the cobordism $F^{-1}([\lambda, \lambda+1])$. Choose any set of regular values of $F$, satisfying $(\Sigma 1)-(\Sigma 3)$ of Definition 6.3.1. Theorem 2.6.5 implies 
that there is an almost good $\left.F\right|_{W}$-gradient $u$ and a ranging pair $\left(\mathcal{V}_{0}, \mathcal{V}_{1}\right)$ for $\left(\left.F\right|_{W}, u\right)$ such that $\|v-u\| \leqslant \epsilon$ and $v=u$ near $\partial W$. We can also assume that $\mathcal{V}_{0} t=\mathcal{V}_{1}$. Then the procedure, described in [15, Construction 4.13], page 1000 defines a bunch of ranging systems for $\left(\left.F\right|_{W}, u\right)$. Extend $u$ to a $t$-invariant $F$-gradient. The BRS constructed is easily extended to a BERS for $(F, u)$.

We mention here an obvious corollary of Theorem $D$ which will be of use in the proof of Theorem E. For a good $f$-gradient $v, c \in \mathbb{R}$ and $x, y \in S(f)$ with ind $x=$ ind $y+1$ we denote by $N_{\geqslant c}(x, y ; v)$ the sum $\sum_{-k \geqslant c} N_{k}(x, y ; v)$.

Corollary 6.4.1. Let $v \in \mathcal{G}_{0}(f)$. Then there are constants $R, Q>0$ such that for every critical points $x, y \in S(f)$ with ind $x=$ ind $y+1$ and every $c \in \mathbb{R}$ we have: $N_{\geqslant c}(x, y ; v) \leqslant R \cdot Q^{-c}$.

6.5. Proof of the Theorem E. By Lemma 4.2.4 find $\epsilon>0$ and $\delta>0$ such that $\omega_{\epsilon}$ is a Morse family, $v$ is an $\omega_{\epsilon}$-gradient and every $\omega$-gradient $u$ with $\|u-v\|<\delta$ is an $\Omega_{\epsilon}$-gradient. Choose some $\xi \in \Omega_{\epsilon}$ with $[\xi] \in H^{1}(M, \mathbf{Q})$ and choose (by Theorem D) a good $\xi$-gradient $u$, satisfying the condition (2) of Theorem $\mathrm{D}$ and such that $\|u-v\|<\delta$. Then $u$ is an $\omega_{\epsilon}$-gradient. By Lemma 4.3 .5 for every $x, y \in S(\omega)$ there is an integral $([\xi],[\omega])$-cone $\Delta$ and $b \in \mathbb{Z}^{m}$ such that

$$
Q(I(\tilde{x}, \tilde{y} ; u)) \subset \Delta+b .
$$

Lemma 5.1.1 implies that there are $A, B$ such that for every $\lambda \in \mathbb{R}$ we have

$$
I(\tilde{x}, \tilde{y} ; u) \cap\{\omega\}^{-1}([\lambda, \infty)) \subset I(\tilde{x}, \tilde{y} ; u) \cap\{\xi\}^{-1}([A \lambda+B, \infty)),
$$

and this together with Corollary 6.4.1 implies the conclusion.

\section{ACKNOWLEDGments}

The author thanks the anonymous referee for many helpful remarks, that have lead to a substantial improvement of the manuscript.

\section{REFERENCES}

[1] V. I. Arnold. Dynamics of intersections. In Analysis, et cetera, pages 77-84. Academic Press, Boston, MA, 1990.

[2] N. Bourbaki. Éléments de mathématique. Fascicule XXVIII. Algèbre commutative. Chapitre 3: Graduations, filtrations et topologies. Chapitre 4: Idéaux premiers associés et décomposition primaire. Actualités Scientifiques et Industrielles, No. 1293. Hermann, Paris, 1961.

[3] Dan Burghelea, Stefan Haller. Dynamics, Laplace transform and spectral geometry. $J$. Topol., 1(1):115-151, 2008, doi: 10.1112/jtopol/jtm005.

[4] P. M. Cohn. Free rings and their relations, volume 19 of London Mathematical Society Monographs. Academic Press, Inc. [Harcourt Brace Jovanovich, Publishers], London, second edition, 1985. 
[5] M. Farber, A. Ranicki. The Morse-Novikov theory of circle-valued functions and noncommutative localization. Tr. Mat. Inst. Steklova, 225(Solitony Geom. Topol. na Perekrest.):381-388, 1999.

[6] François Laudenbach, Carlos Moraga Ferrándiz. A geometric Morse-Novikov complex with infinite series coefficients. C. R. Math. Acad. Sci. Paris, 356(11-12):1222-1227, 2018, doi: 10.1016/j.crma.2018.09.008.

[7] John W. Milnor, James D. Stasheff. Characteristic classes. Princeton University Press, Princeton, N. J.; University of Tokyo Press, Tokyo, 1974. Annals of Mathematics Studies, No. 76.

[8] Marston Morse. The calculus of variations in the large, volume 18 of American Mathematical Society Colloquium Publications. American Mathematical Society, Providence, RI, 1996. Reprint of the 1932 original.

[9] S. P. Novikov. Multivalued functions and functionals. An analogue of the Morse theory. Dokl. Akad. Nauk SSSR, 260(1):31-35, 1981.

[10] S. P. Novikov. Quasiperiodic structures in topology. In Topological methods in modern mathematics (Stony Brook, NY, 1991), pages 223-233. Publish or Perish, Houston, TX, 1993.

[11] Andrei Pajitnov. Modules over some localizations of the ring of Laurent polynomials. Mat. Zametki, 46(5):40-49, 103, 1989, doi: 10.1007/BF01139617.

[12] Andrei Pajitnov. On the Novikov complex for rational Morse forms. Ann. Fac. Sci. Toulouse Math. (6), 4(2):297-338, 1995, http://www.numdam.org/item?id=AFST_ 1995_6_4_2_297_0.

[13] Andrei Pajitnov. Incidence coefficients in the Novikov complex for Morse forms: rationality and exponential growth properties. 1996, https://arxiv.org/abs/dg-ga/9604004.

[14] Andrei Pajitnov. Rationality and exponential growth properties of the boundary operators in the Novikov complex. Math. Res. Lett., 3(4):541-548, 1996, doi: 10.4310/MRL.1996.v3.n4.a12.

[15] Andrei Pajitnov. Rationality of boundary operators in the Novikov complex in general position. Algebra i Analiz, 9(5):92-139, 1997. Translation in St. Petersburg Math. J. 9 (1998), no. 5, 969-1006.

[16] Andrei Pajitnov. $C^{0}$-generic properties of boundary operators in the Novikov complex. In Pseudoperiodic topology, volume 197 of Amer. Math. Soc. Transl. Ser. 2, pages 29-115. Amer. Math. Soc., Providence, RI, 1999, doi: 10.1090/trans2/197/03.

[17] Stephen Smale. On the structure of manifolds. Amer. J. Math., 84:387-399, 1962, doi: $10.2307 / 2372978$.

[18] René Thom. Sur une partition en cellules associée à une fonction sur une variété. $C$. R. Acad. Sci. Paris, 228:973-975, 1949.

[19] Edward Witten. Supersymmetry and Morse theory. J. Differential Geom., 17(4):661692 (1983), 1982, http://projecteuclid.org/euclid.jdg/1214437492.

Received: January 15, 2020, accepted: October 23, 2020.

Andrei Pajitnov

Laboratoire Mathématiques Jean Leray UMR 6629, Université de Nantes, FaCulté des Sciences, 2, Rue de la Houssinière, 44072, NAntes, CedeX

Email: andrei.pajitnov@univ-nantes.fr 